\title{
Automating the POWHEG method in SHERPA
}

\author{
Stefan Höche, ${ }^{a}$ Frank Krauss, $^{b, c}$ Marek Schönherr ${ }^{d}$ and Frank Siegert ${ }^{b, e}$ \\ ${ }^{a}$ Institut für Theoretische Physik, Universität Zürich, \\ CH-8057 Zurich, Switzerland \\ ${ }^{b}$ Institute for Particle Physics Phenomenology, Durham University, \\ Durham DH1 3LE, U.K. \\ ${ }^{c} \mathrm{PH}-\mathrm{TH}, \mathrm{CERN}$, \\ CH-1211 Geneva 23, Switzerland \\ ${ }^{d}$ Institut für Kern- und Teilchenphysik, Technische Universität Dresden, \\ D-01062, Dresden, Germany \\ ${ }^{e}$ Department of Physics \& Astronomy, University College London, \\ London WC13 6BT, U.K. \\ E-mail: shoeche@slac.stanford.edu, frank.krauss@durham.ac.uk, \\ marek.schoenherr@tu-dresden.de, frank.siegert@cern.ch
}

Abstract: A new implementation of the Powheg method [1, 2] into the Monte-Carlo event generator SHERPA [3, 4] is presented, focusing on processes with a simple colour structure. Results for a variety of reactions, namely $e^{+} e^{-} \rightarrow$ hadrons, deep-inelastic leptonnucleon scattering, hadroproduction of single vector bosons and of vector boson pairs as well as the production of Higgs bosons in gluon fusion serve as test cases for the successful realisation. The algorithm is highly automated such that for other processes with simple colour topology only virtual matrix elements need to be provided.

KEYWORDS: QCD Phenomenology

ARXIV EPRINT: 1008.5399 


\section{Contents}

1 Introduction $\quad 1$

2 Parton showers and the POWHEG method 4

2.1 Decomposition of real-emission cross sections 4

2.2 Construction of the parton shower $\quad 6$

2.3 Correcting parton showers with matrix elements 8

2.4 Approximate NLO cross sections 9

$\begin{array}{ll}2.5 & \text { The Powheg method and its accuracy } \\ \end{array}$

3 Realisation of the Powheg method in the Sherpa Monte Carlo 12

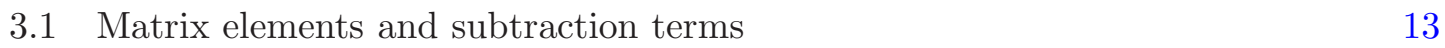

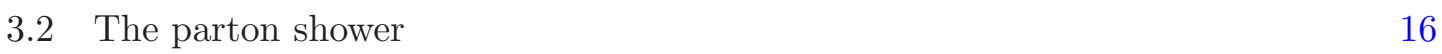

$\begin{array}{lll}3.2 .1 & \text { Ordering parameters and splitting functions } & 16\end{array}$

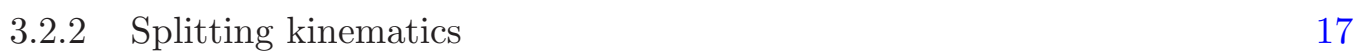

$\begin{array}{lll}3.3 & \text { Automatic identification of Born zeros } & 18\end{array}$

4 Results $\quad 19$

$\begin{array}{lll}4.1 & \text { Process listing } & 19\end{array}$

$\begin{array}{lll}\text { 4.1.1 Jet production in } e^{+} e^{-} \text {collisions } & 19\end{array}$

$\begin{array}{lll}\text { 4.1.2 Deep-inelastic lepton-nucleon scattering } & 19\end{array}$

$\begin{array}{lll}\text { 4.1.3 Drell-Yan lepton pair production } & 20\end{array}$

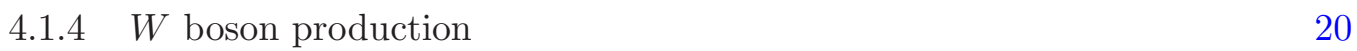

4.1.5 Higgs boson production through gluon-gluon fusion 20

$\begin{array}{lll}4.1 .6 & Z \text {-pair production } & 21\end{array}$

$\begin{array}{lll}\text { 4.1.7 } & W^{+} W^{-} \text {-production } & 21\end{array}$

$\begin{array}{lll}4.2 & \text { Tests of internal consistency } & 22\end{array}$

4.3 Comparison with tree-level matrix-element parton-shower merging 25

$\begin{array}{ll}4.4 & \text { Comparison with experimental data }\end{array}$

4.5 Comparison with existing Powheg results 32

5 Conclusions and outlook $\quad 35$

\section{Introduction}

Higher-order QCD corrections by now form an important ingredient to many phenomenological studies and experimental analyses at both the Tevatron and the LHC. Their impact has been similarly important for various studies of HERA and LEP data. Calculations invoking such corrections, typically at next-to leading order (NLO) in the perturbative 
expansion in the strong coupling $\alpha_{s}$, and in very few cases also at next-to-next-to leading order (NNLO) accuracy, have been used for a wide range of precision tests of our understanding of QCD and the Standard Model. They are also important for the subtraction of backgrounds in searches for new physics. When being compared to such calculations, experimental measurements are usually corrected for detector effects, while the perturbative result is corrected for hadronisation. Only after performing these corrections, theoretical predictions and experimental data are on the same footing. Hadronisation corrections are typically determined by using multi-purpose Monte-Carlo event generators, such as Pythia/Pythia 8 [5, 6], Herwig/Herwig++ [7, 8], or, more recently, Sherpa [3, 4]. In the past two decades, such event generators have been the workhorses of particle physics phenomenology, including in their simulation many features in addition to the perturbative aspects of a collision, such as hadronisation, the underlying event, hadron decays etc.. But, to describe perturbative QCD, they typically relied on leading-order (LO) matrix elements only, combined with parton showers, which in turn model QCD radiation effects in a leading logarithmic approximation.

Improvements to this approximation can be obtained through merging methods, pioneered in [9-11], and further worked out in different varieties at different accuracies and for different parton showers, e.g. in [12-16]. In this merging approach, tree-level matrix elements for processes with different jet multiplicities are combined with parton showers, avoiding problems related to double counting of emissions. Lately, a new formulation has been proposed which can be proved to preserve the formal accuracy of the parton shower, independent of the process under consideration [17]. ${ }^{1}$ Despite varying degrees of formal accuracy amongst the various methods, their respective predictions tend to agree on a level expected from such improved leading order perturbation theory $[19,20]$. In most cases the approach leads to a dramatic improvement in the description of hard QCD radiation, which makes it a state-of-the-art tool for many analyses, that depend on the shape of distributions related to hard $\mathrm{QCD}$ radiation.

However sophisticated at modelling multi-jet topologies, the above methods lack the precision of higher-order calculations regarding the overall normalisation, i.e. the cross section of the inclusive process under consideration. To accurately estimate uncertainties intrinsic to perturbative calculations, which manifest themselves for instance in uncertainties related to choices of renormalisation and factorisation scales, full next-to leading order corrections are a sine qua non.

So far, two different methods to achieve the systematic inclusion of complete NLO corrections for a fixed multiplicity have not only been described but also implemented, asserting their practicality. The first one has been dubbed Mc@NLO [21]. By now it has been applied to a variety of processes using the framework of both the HeRWIG and the Pythia [26] event generators, proving its versatility. It effectively relies on using the parton shower to perform the subtraction of infrared divergences in the real-emission part of NLO corrections, resulting in a separation of the event generation into two parts,

\footnotetext{
${ }^{1} \mathrm{~A}$ similar procedure, although restricted to the case of $e^{+} e^{-}$-annihilation into hadrons was also presented in $[18]$.
} 
one starting from the Born kinematics of the hard process, the other starting with realemission kinematics, i.e. including an extra parton in the final state. The weights for these two samples are adjusted accordingly and yield the total cross section and the hardest emission, correct to first order in $\alpha_{s}$. However, this technique may produce a number of events with a negative weight. The second method to include NLO corrections into parton showers is known as the Powheg technique $[1,2]$. In contrast to MC@NLO, it can ensure that only events with positive weights are generated. To achieve this, the simulation starts with a Born-level event, reweighted to include the full NLO correction, i.e. including virtual and real corrections, integrated over the real-emission subspace. The first emission is then produced using the exact real-emission matrix element, thus providing not only the correct differential cross section, but also the correct radiation pattern to first order in $\alpha_{s}$. The big advantage of this technique is that it can straightforwardly be implemented in a showerindependent way, which has been used to some extent in [27-32], where different processes have been treated in the framework of PyтнiA and/or HeRwiG. In addition, there have been some implementations in the framework of HeRwiG++ [33-37]. ${ }^{2}$ In fact, because the Powheg method is very similar to traditional matrix-element corrections [38-43], it is relatively simple to implement in a process-independent way and thus very well suited for the systematic inclusion of NLO QCD corrections to arbitrary processes. This is reflected by the fact that a corresponding toolbox has already been advertised in [44].

Obviously, the ultimate goal is to have a multi-jet merging prescription, accurate at $\mathrm{NLO}$, and to simultaneously respect the logarithmic accuracy of the parton shower. A first step into this direction has been presented for the case of $e^{+} e^{-}$annihilation into hadrons in [45]. An alternative approach was suggested in [46], extending the method of [17] to NLO accuracy for the core interaction. This second method relies on the PowHEg technique to ensure the NLO cross section of the core process. Parallel development has brought about a similar algorithm, which will be reported shortly [47] and which is based on the generic POWHEG implementation presented here.

The outline of this paper is as follows: In section 2 the connection between the POWHEG method and parton showers will be detailed, introducing also the notation used in this publication. The possibility to implement the POWHEG technique on top of an existing parton shower will be discussed. Section 3 briefly introduces the SHERPA event generator, which sets the framework for this study. Those parts which are relevant for the PowhEg implementation will be presented in more detail. Due to the overall setup of SHERPA, incorporating matrix element generators capable of producing the real correction terms for a given process and the corresponding differential and integrated subtraction terms in the CataniSeymour dipole subtraction scheme [48, 49], the only missing bit of the NLO calculation driving the POWHEG method are the virtual contributions, which have been made accessible through interfaces to BlackHat [50-52] and MCFM [53]. It is the first time that the PowHEg method has been automated using dipole subtraction rather than the FrixioneKunszt-Signer method $[54,55]$. The overall quality of the implementation will be exempli-

\footnotetext{
${ }^{2}$ It is worth stressing, that in order to ensure formal accuracy a truncated shower must be used, as pointed out in the original publications proposing the method. This option is not available for all parton shower algorithms that have been used in the actual implementations.
} 
fied in a number of characteristic processes in section 4, including the hitherto unpublished case of $W$-pair production in hadronic collisions. Section 5 contains our conclusions.

\section{Parton showers and the POWHEG method}

In this publication, the POWHEG method is reinterpreted as an advanced matrix-element correction technique for standard parton showers. The following section introduces the necessary notation and outlines the parallels between the POWHEG method and traditional matrix-element corrections. The starting point of the discussion is the factorisation theorem underlying the specific parton-shower model, like the DGLAP equation [56-59], the colourdipole model [60-62], Catani-Seymour factorisation [48, 49] or antenna factorisation [6366]. Except in collinear factorisation, the splitting functions of the parton shower depend on (at least) one additional parton, which is often referred to as the "spectator". In order to make this connection explicit, the notation of a dipole-like factorisation is adopted, which is sufficiently general to discuss all relevant features of the PowHEG method and its implementation into the SHERPA event generator.

\subsection{Decomposition of real-emission cross sections}

In the following, sets of $n$ particles in a $2 \rightarrow(n-2)$ process will summarily be denoted by $\{\vec{a}\}=\left\{a_{1}, \ldots, a_{n}\right\}$, and the particles will be specified through their flavours $\{\vec{f}\}=$ $\left\{f_{1}, \ldots, f_{n}\right\}$ and momenta $\{\vec{p}\}=\left\{p_{1}, \ldots, p_{n}\right\}$. The generic expression for a fully differential Born-level cross section in a scattering process with $(n-2)$ final-state particles can be written as a sum over all contributing flavour combinations as

$$
\mathrm{d} \sigma_{B}(\{\vec{p}\})=\sum_{\{\vec{f}\}} \mathrm{d} \sigma_{B}(\{\vec{a}\}), \quad \text { where } \quad \mathrm{d} \sigma_{B}(\{\vec{a}\})=\mathrm{d} \Phi_{B}(\{\vec{p}\}) \mathrm{B}(\{\vec{a}\}),
$$

The individual terms in the sum are given by

$$
\begin{aligned}
\mathrm{B}(\{\vec{a}\}) & =\mathcal{L}(\{\vec{a}\}) \mathcal{B}(\{\vec{a}\}), & \mathcal{B}(\{\vec{a}\}) & =\frac{1}{F(\{\vec{p}\})} \frac{1}{S(\{\vec{f}\})}\left|\mathcal{M}_{B}\right|^{2}(\{\vec{a}\}), \\
\mathrm{d} \Phi_{B}(\{\vec{p}\}) & =\frac{\mathrm{d} x_{1}}{x_{1}} \frac{\mathrm{d} x_{2}}{x_{2}} \mathrm{~d} \Phi_{B}(\{\vec{p}\}), & \mathcal{L}\left(\{\vec{a}\} ; \mu^{2}\right) & =x_{1} f_{f_{1}}\left(x_{1}, \mu^{2}\right) x_{2} f_{f_{2}}\left(x_{2}, \mu^{2}\right) .
\end{aligned}
$$

Here, $\left|\mathcal{M}_{B}\right|^{2}(\{\vec{a}\})$ denotes the partonic matrix element squared, with all factors due to averaging over initial state quantum numbers such as spin or colours absorbed into it, and $\mathrm{d} \Phi_{B}(\{\vec{p}\})$ is the corresponding differential $n$-particle partonic phase-space element; $S(\{\vec{f}\})$ is the symmetry factor due to identical flavours associated to the partonic subprocess, while $F(\{\vec{p}\})$ denotes the flux factor and $\mathcal{L}$ is the parton luminosity given by the corresponding parton distribution functions (PDFs). In the case of leptonic initial states, ignoring QED initial state radiation, the parton distribution functions $f\left(x, \mu^{2}\right)$ are replaced by $\delta(1-x)$.

In a similar fashion, the real-emission part of the QCD next-to-leading order cross section can be written as a sum, this time over parton configurations $\left\{a_{1}, \ldots, a_{n+1}\right\}$, i.e. including one additional parton. A corresponding subprocess cross section reads

$$
\mathrm{d} \sigma_{R}(\{\vec{a}\})=\mathrm{d} \Phi_{R}(\{\vec{p}\}) \mathrm{R}(\{\vec{a}\}) .
$$


At this point, it is helpful to introduce a notation for mappings from real-emission parton configurations to Born parton configurations. Such mappings combine the partons $a_{i}$ and $a_{j}$ into a common "mother" parton $a_{\widetilde{\imath \jmath}}$, in the presence of the spectator $a_{k}$ by defining a new flavour $f_{\widetilde{\imath \jmath}}$ and by redefining the particle momenta. To be specific,

$$
b_{i j, k}(\{\vec{a}\})=\left\{\begin{array}{c}
\{\vec{f}\} \backslash\left\{f_{i}, f_{j}\right\} \cup\left\{f_{\widetilde{\imath \jmath}}\right\} \\
\{\vec{p}\} \rightarrow\{\overrightarrow{\tilde{p}}\}
\end{array}\right.
$$

The flavour of the "mother" parton, $f_{\widetilde{\imath \jmath}}$, is thereby fixed unambiguously by the QCD interactions, while the flavour of the spectator, $f_{k}$, remains unaltered, cf. also [48, 49]. The momentum map guarantees that all partons are kept on their mass shell.

Conversely, any Born parton configuration and a related branching process $\widetilde{\imath \jmath}, \tilde{k} \rightarrow i j, k$ determine the parton configuration of a real-emission subprocess as

$$
r_{\widetilde{\imath \jmath}, \tilde{k}}\left(f_{i}, \Phi_{R \mid B} ;\{\vec{a}\}\right)=\left\{\begin{array}{c}
\{\vec{f}\} \backslash\left\{f_{\widetilde{\imath \jmath}}\right\} \cup\left\{f_{i}, f_{j}\right\} \\
\{\overrightarrow{\tilde{p}}\} \rightarrow\{\vec{p}\}
\end{array} .\right.
$$

The radiative variables $\Phi_{R \mid B}$ are thereby employed to turn the $n$-parton momentum configuration into an $\mathrm{n}+1$-parton momentum configuration using the inverse of the phase-space map defined by eq. (2.4). The flavour $f_{j}$ is again determined unambiguously by the QCD interactions. Here, also two obvious generalisations of eq. (2.4) shall be defined, $b_{i j, k}(\{\vec{f}\})$ and $b_{i j, k}(\{\vec{p}\})$, which act on the parton flavours and on the parton momenta only. Correspondingly, such generalisations exist for eq. (2.5).

In the soft and collinear limits, the partonic matrix element squared, $\mathcal{R}(\{\vec{a}\})$, can be decomposed as a sum of terms $\mathcal{D}_{i j, k}(\{\vec{a}\})$,

$$
\mathcal{R}(\{\vec{a}\}) \rightarrow \sum_{\{i, j\}} \sum_{k \neq i, j} \mathcal{D}_{i j, k}(\{\vec{a}\}) .
$$

These terms factorise into a Born-level term and a universal splitting kernel, encoding the transition of $a_{\widetilde{\imath \jmath}}$ to $a_{i}$ and $a_{j}[48,49]$. The splitting is associated with a universal procedure for factorising the phase-space integral into a Born level part and a one-particle radiative phase space.

$$
\mathrm{d} \Phi_{R}(\{\vec{p}\})=\mathrm{d} \Phi_{B}\left(b_{i j, k}(\{\vec{p}\})\right) \mathrm{d} \Phi_{R \mid B}^{i j, k}(\{\vec{p}\}) .
$$

The existence of universal decompositions like in eq. (2.6) forms the basis of subtraction methods like the Catani-Seymour dipole subtraction [48, 49], antenna subtraction [63-66], or the subtraction method of Frixione, Kunszt, and Signer [54, 55]. It also serves as starting point for the construction of parton shower algorithms [67-69], which aim at approximating parton emissions in the collinear and soft limits of the radiative phase space, to resum the associated large logarithms, cf. section 2.2.

However, it is important to stress that, also away from the infrared limits, $\mathcal{R}(\{\vec{a}\})$ can be decomposed into a number of terms $\mathcal{R}_{i j, k}$ analogous to $\mathcal{D}_{i j, k}$ through

$$
\mathcal{R}_{i j, k}(\{\vec{a}\}):=\rho_{i j, k}(\{\vec{a}\}) \mathcal{R}(\{\vec{a}\}), \quad \text { where } \quad \rho_{i j, k}(\{\vec{a}\})=\frac{\mathcal{D}_{i j, k}(\{\vec{a}\})}{\sum_{\{m, n\}} \sum_{l \neq m, n} \mathcal{D}_{m n, l}(\{\vec{a}\})} .
$$


Equation (2.3) can now be rewritten as a sum of trivially factorised contributions

$$
\mathrm{d} \sigma_{R}(\{\vec{a}\})=\sum_{\{i, j\}} \sum_{k \neq i, j} \mathrm{~d} \sigma_{B}\left(b_{i j, k}(\{\vec{a}\})\right) \mathrm{d} \sigma_{R \mid B}^{i j, k}(\{\vec{a}\})
$$

where

$$
\mathrm{d} \sigma_{R \mid B}^{i j, k}(\{\vec{a}\})=\mathrm{d} \Phi_{R \mid B}^{i j, k}(\{\vec{p}\}) \frac{\mathrm{R}_{i j, k}(\{\vec{a}\})}{\mathrm{B}\left(b_{i j, k}(\{\vec{a}\})\right)} .
$$

and $\mathrm{R}_{i j, k}(\{\vec{a}\})=\mathcal{L}(\{\vec{a}\}) \mathcal{R}_{i j, k}(\{\vec{a}\})$. These equations are key ingredients to understanding and implementing the Powheg method.

\subsection{Construction of the parton shower}

Due to the non-Abelian nature of $\mathrm{QCD}$, the terms $\mathcal{D}_{i j, k}$ in eq. (2.6) in general do not factorise on the level of squared matrix elements, including all colour contributions. To arrive at a practical model for a parton shower, sub-leading colour configurations are therefore neglected, which leads to an assumed factorisation on the level of squared matrix elements. In the infrared limits one can then write

$$
\mathcal{D}_{i j, k}(\{\vec{a}\}) \rightarrow \mathcal{B}\left(b_{i j, k}(\{\vec{a}\})\right) \frac{S\left(b_{i j, k}(\{\vec{f}\})\right)}{S(\{\vec{f}\})} \frac{1}{2 p_{i} p_{j}} 8 \pi \alpha_{s} \mathcal{K}_{i j, k}\left(p_{i}, p_{j}, p_{k}\right),
$$

where the set of momenta $b_{i j, k}(\{\vec{p}\})$ is determined by the phase-space map of the partonshower model. ${ }^{3}$ The quantities $\mathcal{K}_{i j, k}$ are the parton-shower evolution kernels, which depend on the parton flavours $f_{i}, f_{j}$ and $f_{k}$ and on the radiative phase space. The denominator factor $2 p_{i} p_{j}$ or any linearly dependent quantity is usually used to define the parton shower evolution variable, in the following denoted by $t$.

Using the above model, the parton-shower approximation of eq. (2.10) can be derived as

$$
\mathrm{d} \sigma_{R \mid B}^{(\mathrm{PS}) i j, k}(\{\vec{a}\})=\mathrm{d} \Phi_{R \mid B}^{i j, k}(\{\vec{p}\}) \frac{S\left(b_{i j, k}(\{\vec{f}\})\right)}{S(\{\vec{f}\})} \frac{1}{2 p_{i} p_{j}} 8 \pi \alpha_{s} \mathcal{K}_{i j, k}\left(p_{i}, p_{j}, p_{k}\right) \frac{\mathcal{L}(\{\vec{a}\})}{\mathcal{L}\left(b_{i j, k}(\{\vec{a}\})\right)} .
$$

Particles produced in the parton shower are resolved at a certain evolution scale and can therefore be distinguished from particles at higher and lower scales. At most the two particles $a_{i}$ and $a_{j}$, emerging from the same splitting process, can be seen as identical. Hence, the ratio of symmetry factors in eq. (2.10) changes to

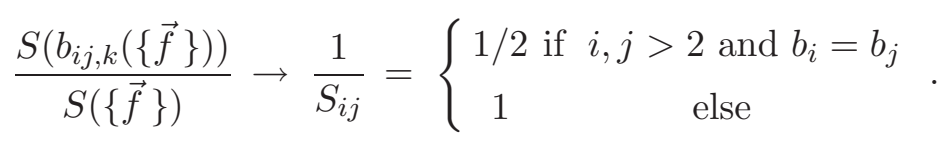

The integral over the radiative phase space can be written as

$$
\mathrm{d} \Phi_{R \mid B}^{i j, k}(\{\vec{p}\})=\frac{1}{16 \pi^{2}} \mathrm{~d} t \mathrm{~d} z \frac{\mathrm{d} \phi}{2 \pi} J_{i j, k}(t, z, \phi),
$$

\footnotetext{
${ }^{3}$ Note that here only parton showers with local energy-momentum conservation are considered. Therefore, the phase-space maps $\{\vec{p}\}_{\mathrm{R}} \rightarrow\{\vec{p}\}_{\mathrm{B}}$ exist.
} 
with $t$ the evolution variable, $z$ the splitting variable, and $\phi$ an azimuthal angle. Here, $J$ denotes the Jacobian factor, that potentially arises due to the transformation of variables. Equation (2.12) thus becomes

$$
\mathrm{d} \sigma_{R \mid B}^{(\mathrm{PS}) i j, k}(\{\vec{a}\})=\frac{\mathrm{d} t}{t} \mathrm{~d} z \frac{\mathrm{d} \phi}{2 \pi} \frac{\alpha_{s}}{2 \pi} \frac{1}{S_{i j}} J_{i j, k}(t, z, \phi) \mathcal{K}_{i j, k}(t, z, \phi) \frac{\mathcal{L}(\{\vec{a}\} ; t)}{\mathcal{L}\left(b_{i j, k}(\{\vec{a}\}) ; t\right)} .
$$

The assignment of the mother parton, the spectator and the underlying Born process can now be assumed to be fixed. Then, the sum runs over all possible real-emission configurations originating from this particular Born-level state instead. Furthermore, assuming independence of the individual emissions, i.e. Poissonian statistics, this leads to the constrained no-branching probability of the parton-shower model $[70,71]$ between the two scales $t^{\prime \prime}$ and $t^{\prime}$

$$
\begin{aligned}
\Delta_{\widetilde{\imath \jmath}, \tilde{k}}^{(\mathrm{PS})}\left(t^{\prime}, t^{\prime \prime} ;\{\vec{a}\}\right)=\exp \left\{-\sum_{f_{i}=q, g}\right. & \int_{t^{\prime}}^{t^{\prime \prime}} \frac{\mathrm{d} t}{t} \int_{z_{\min }}^{z_{\max }} \mathrm{d} z \int_{0}^{2 \pi} \frac{\mathrm{d} \phi}{2 \pi} J_{i j, k}(t, z, \phi) \\
& \left.\times \frac{1}{S_{i j}} \frac{\alpha_{s}}{2 \pi} \mathcal{K}_{i j, k}(t, z, \phi) \frac{\mathcal{L}\left(r_{\widetilde{\imath}, \tilde{k}}\left(f_{i}, t, z, \phi ;\{\vec{a}\}\right) ; t\right)}{\mathcal{L}(\{\vec{a}\} ; t)}\right\} .
\end{aligned}
$$

It is worth noting that eq. (2.16) depends on the underlying Born process, since the flavour and momentum of the spectator enter as arguments of $J_{i j, k}$ and $\mathcal{K}_{i j, k}$. The ratio of $\mathcal{L}$ in eq. (2.16) accounts for a potential change of the parton luminosity when integrating over the initial-state phase space. ${ }^{4}$ Note that the partons $\{\vec{a}\}$ in eq. (2.16) denote a Born-level set, while in (2.12) and (2.15) $\{\vec{a}\}$ denote a set of partons at real-emission level.

Using the definition

$$
\Delta^{(\mathrm{PS})}\left(t_{0}, \mu^{2} ;\{\vec{a}\}\right)=\prod_{\{\widetilde{\imath}, \tilde{k}\}} \Delta_{\widetilde{\imath}, \tilde{k}}^{(\mathrm{PS})}\left(t_{0}, \mu^{2} ;\{\vec{a}\}\right)
$$

the total cross section in the parton-shower approximation reads

$$
\begin{aligned}
& \sigma_{B}=\sum_{\{\vec{f}\}} \int \mathrm{d} \Phi_{B}(\{\vec{p}\}) \mathrm{B}(\{\vec{a}\})\left[\Delta^{(\mathrm{PS})}\left(t_{0}, \mu^{2} ;\{\vec{a}\}\right)\right. \\
&+\sum_{\{\widetilde{\imath}, \tilde{k}\}} \sum_{f_{i}=q, g} \int_{t_{0}}^{\mu^{2}} \frac{\mathrm{d} t}{t} \int_{z_{\min }}^{z_{\max }} \mathrm{d} z \int_{0}^{2 \pi} \frac{\mathrm{d} \phi}{2 \pi} J_{i j, k}(t, z, \phi) \\
&\left.\quad \times \frac{1}{S_{i j}} \frac{\alpha_{s}}{2 \pi} \mathcal{K}_{i j, k}(t, z, \phi) \frac{\mathcal{L}\left(r_{\widetilde{\imath}, \tilde{k}}\left(f_{i}, t, z, \phi ;\{\vec{a}\}\right) ; t\right)}{\mathcal{L}(\{\vec{a}\} ; t)} \Delta^{(\mathrm{PS})}\left(t, \mu^{2} ;\{\vec{a}\}\right)\right] .
\end{aligned}
$$

The scale $t_{0}$ acts as the infrared cutoff of the parton shower. Simple inspection shows that the sum in the square bracket equals unity, since the second term can be written as

$$
\int_{t_{0}}^{\mu^{2}} \mathrm{~d} t \frac{\mathrm{d} \Delta^{(\mathrm{PS})}\left(t, \mu^{2} ;\{\vec{a}\}\right)}{\mathrm{d} t} .
$$

\footnotetext{
${ }^{4}$ Note that, depending on the parton shower model, the $x_{i}$ do not necessarily fulfil the relation $x_{i}=$ $\tilde{x}_{i} / z[72,73]$.
} 
This makes the probabilistic properties of the parton shower explicit. At the same time it also shows that this unitarity leads to the cross section in standard parton-shower Monte Carlos to be exactly the respective leading-order cross section. In order to evaluate the formal accuracy of the description of the radiation pattern, induced by the second term in the square bracket - the first term encodes the probability that there is no resolvable emission off the Born-level configuration - a corresponding observable must be introduced. This complicates the discussion somewhat and is therefore postponed to sections 2.4 and 2.5.

\subsection{Correcting parton showers with matrix elements}

The aim of this section is to devise a simple method for reinstating $\mathcal{O}\left(\alpha_{s}\right)$ accuracy in the emission pattern of the parton shower, i.e. the hardest emission in the parton shower should follow the distribution given by the corresponding real-emission matrix element. Loosely speaking, the key idea is to replace the splitting kernels $\mathcal{K}$ with the ratio of realemission and Born-level matrix elements. Thus, instead of the splitting kernels, this ratio is exponentiated in the Sudakov form factor and employed in simulating the splitting.

Comparing eqs. (2.10) and (2.12), a corresponding factor correcting $\mathcal{K}$ to resemble $\mathrm{R} / \mathrm{B}$ can be easily identified. Using eq. (2.13) it reads

$$
w_{i j, k}(\{\vec{a}\})=\frac{\mathrm{d} \sigma_{R \mid B}^{i j, k}(\{\vec{a}\})}{\mathrm{d} \sigma_{R \mid B}^{(\mathrm{PS}) i j, k}(\{\vec{a}\})}=\frac{2 p_{i} p_{j}}{8 \pi \alpha_{s}} \frac{S(\{\vec{f}\})}{S\left(b_{i j, k}(\{\vec{f}\})\right)} \frac{\rho_{i j, k}(\{\vec{a}\}) \mathcal{R}(\{\vec{a}\})}{\mathcal{B}\left(b_{i j, k}(\{\vec{a}\})\right) \mathcal{K}_{i j, k}(\{\vec{a}\})} .
$$

Employing the parton-shower approximation, eq. (2.11), to replace $\rho_{i j, k}$ yields

$$
w(\{\vec{a}\})=\left[\sum_{\{m, n\}} \sum_{l \neq m, n} \frac{S\left(b_{m n, l}(\{\vec{f}\})\right)}{S(\{\vec{f}\})} \frac{\mathcal{B}\left(b_{m n, l}(\{\vec{a}\})\right)}{\mathcal{R}(\{\vec{a}\})} \frac{8 \pi \alpha_{s}}{2 p_{m} p_{n}} \mathcal{K}_{m n, l}(\{\vec{a}\})\right]^{-1} .
$$

Note that this implies a corrective weight, which is actually splitter-spectator independent. Correcting the parton shower to the full matrix element can thus be achieved through the following algorithm:

1. Determine an overestimate for eq. (2.21), i.e. find a set of $W_{\widetilde{\imath}, f_{i}}(\{\vec{f}\})$, such that $w\left(r_{\widetilde{\imath}, \tilde{k}}\left(f_{i}, \Phi_{R \mid B} ;\{\vec{a}\}\right)\right) \leq W_{\widetilde{\imath \jmath}, f_{i}}(\{\vec{f}\})$ for all $\tilde{k}$ and throughout the real-emission phase space.

2. Replace the parton shower splitting kernels $\mathcal{K}_{i j, k}$ by $W_{\widetilde{\imath}, f_{i}}(\{\vec{f}\}) \mathcal{K}_{i j, k}$.

3. Accept parton-shower branchings with probability $w\left(r_{\widetilde{\imath}, \tilde{k}}\left(f_{i}, \Phi_{R \mid B} ;\{\vec{a}\}\right)\right) / W_{\widetilde{\imath}, f_{i}}(\{\vec{f}\})$.

It is straightforward to show that the constrained no-branching probability of such a matrixelement corrected parton shower reads

$$
\begin{aligned}
\Delta_{\widetilde{\imath}, \tilde{k}}^{(\mathrm{ME})}\left(t^{\prime}, t^{\prime \prime} ;\{\vec{a}\}\right)=\exp \left\{-\sum_{f_{i}=q, g}\right. & \frac{1}{16 \pi^{2}} \int_{t^{\prime}}^{t^{\prime \prime}} \mathrm{d} t \int_{z_{\min }}^{z_{\max }} \mathrm{d} z \int_{0}^{2 \pi} \frac{\mathrm{d} \phi}{2 \pi} J_{i j, k}(t, z, \phi) \\
& \left.\times \frac{1}{S_{i j}} \frac{S\left(r_{\widetilde{\imath}, \tilde{k}}\left(f_{i} ;\{\vec{f}\}\right)\right)}{S(\{\vec{f}\})} \frac{\mathrm{R}_{i j, k}\left(r_{\widetilde{\imath}, \tilde{k}}\left(f_{i}, t, z, \phi ;\{\vec{a}\}\right)\right)}{\mathrm{B}(\{\vec{a}\})}\right\} .
\end{aligned}
$$


The ratio $\mathrm{R} / \mathrm{B}$ in eq. (2.22) coincides with the ratio in the original publications presenting the Powheg method. In the relatively simple cases treated so far $[1,2,27-32]$, the various symmetry factors in the equation above cancel and can be neglected. For more complicated flavour structures this factor may differ from one and therefore must be retained.

Employing again the definition of eq. (2.17), but this time for the Sudakov form factor $\Delta^{(\mathrm{ME})}$ constructed from the ratio $\mathrm{R} / \mathrm{B}$ yields the cross section in the matrix element improved parton shower approximation. It reads

$$
\begin{aligned}
\sigma_{B}=\sum_{\{\vec{f}\}} \int \mathrm{d} & \Phi_{B}(\{\vec{p}\}) \mathrm{B}(\{\vec{a}\})\left[\Delta^{(\mathrm{ME})}\left(t_{0}, \mu^{2} ;\{\vec{a}\}\right)\right. \\
+ & \sum_{\{\widetilde{\imath}, \tilde{k}\}} \sum_{f_{i}=q, g} \frac{1}{16 \pi^{2}} \int_{t_{0}}^{\mu^{2}} \mathrm{~d} t \int_{z_{\min }}^{z_{\max }} \mathrm{d} z \int_{0}^{2 \pi} \frac{\mathrm{d} \phi}{2 \pi} J_{i j, k}(t, z, \phi) \\
& \left.\times \frac{1}{S_{i j}} \frac{S\left(r_{\widetilde{\imath}, \tilde{k}}\left(f_{i} ;\{\vec{f}\}\right)\right)}{S(\{\vec{f}\})} \frac{\mathrm{R}_{i j, k}\left(r_{\widetilde{\imath}, \tilde{k}}\left(f_{i}, t, z, \phi ;\{\vec{a}\}\right)\right)}{\mathrm{B}(\{\vec{a}\})} \Delta^{(\mathrm{ME})}\left(t, \mu^{2} ;\{\vec{a}\}\right)\right] .
\end{aligned}
$$

Again, the term in the square bracket equals one and thus reflects the probabilistic nature of this approach. Consequently, in the matrix-element improved parton-shower approximation the total cross section is given by the Born cross section, although the radiation pattern has improved. For a detailed discussion of the real-emission term see section 2.5.

\subsection{Approximate NLO cross sections}

In the previous two sections it has become clear that the total cross section of events simulated in a parton-shower Monte-Carlo is determined by the "seed" cross section, typically computed at Born level. While matrix-element improvement of the naive parton-shower picture will lead to radiation patterns which are accurate to $\mathcal{O}\left(\alpha_{s}\right)$, the total cross section of the event sample and any observable that can be defined at Born level will still be given by the respective leading-order expression. To allow for a simulation with next-to-leading order accuracy, including the cross section of the event sample, a prescription to assign a corresponding weight and multiplicity of the seed event must be found.

The solution is to replace the original Born-level matrix element with a modified one $[1,2]$, denoted by $\overline{\mathrm{B}}$,

$$
\mathrm{d} \sigma_{B}(\{\vec{a}\}) \rightarrow \mathrm{d} \sigma_{\bar{B}}(\{\vec{a}\}):=\mathrm{d} \Phi_{B}(\{\vec{p}\}) \overline{\mathrm{B}}(\{\vec{a}\})
$$

such that the "seed" cross section, $\mathrm{d} \sigma_{\bar{B}}$, integrates to the full NLO result. When constructing such an NLO-weighted differential cross section for the Born configuration, certain approximations must be made, since NLO cross sections have two contributions, one with Born-like kinematics and one with real-emission like kinematics, both of which exhibit divergent structures. The value of a given infrared and collinear safe observable, $O$, computed at NLO, is given in terms of the Born term $\mathrm{B}$, the real emission term $\mathrm{R}$, and 
the virtual contribution (including the collinear counter-terms), denoted by $\tilde{\mathrm{V}}$, as

$$
\begin{gathered}
\langle O\rangle^{(\mathrm{NLO})=} \sum_{\{\vec{f}\}} \int \mathrm{d} \Phi_{B}(\{\vec{p}\})[\mathrm{B}(\{\vec{a}\})+\tilde{\mathrm{V}}(\{\vec{a}\})] O(\{\vec{p}\}) \\
+\sum_{\{\vec{f}\}} \int \mathrm{d} \Phi_{R}(\{\vec{p}\}) \mathrm{R}(\{\vec{a}\}) O(\{\vec{p}\}) .
\end{gathered}
$$

It is obvious that the real-emission contribution cannot be simply combined with the Born and virtual terms, as it depends on different kinematics. In the following, the solution of this problem in the framework of the PowHEG method is outlined.

In order to compute eq. (2.25) in a Monte-Carlo approach, subtraction terms, rendering the real emission finite in $D=4$ space-time dimensions are introduced. Corresponding integrated subtraction terms regularise the infrared divergences of the virtual terms. In the dipole subtraction method $[48,49]$, the equation above can then be written as

$$
\begin{aligned}
\langle O\rangle^{(\mathrm{NLO})}= & \sum_{\{\vec{f}\}} \int \mathrm{d} \Phi_{B}(\{\vec{p}\})[\mathrm{B}(\{\vec{a}\})+\tilde{\mathrm{V}}(\{\vec{a}\})+\mathrm{I}(\{\vec{a}\})] O(\{\vec{p}\}) \\
& \quad+\sum_{\{\vec{f}\}} \int \mathrm{d} \Phi_{R}(\{\vec{p}\})\left[\mathrm{R}(\{\vec{a}\}) O(\{\vec{p}\})-\sum_{\{i, j\}} \sum_{k \neq i, j} \mathrm{~S}_{i j, k}(\{\vec{a}\}) O\left(b_{i j, k}(\{\vec{p}\})\right)\right] .
\end{aligned}
$$

Note that each $\mathrm{S}_{i j, k}$ defines a separate phase-space map and that the observable $O$ in the last term depends on $b_{i j, k}(\{\vec{p}\})$, rather than $\{\vec{p}\}$, which is a crucial feature of the subtraction procedure. The real and integrated subtraction terms $\mathrm{S}_{i j, k}(\{\vec{a}\})$ and $\mathrm{I}(\{\vec{a}\})$ fulfil the relation

$$
\sum_{\{\vec{f}\}} \int \mathrm{d} \Phi_{B}(\{\vec{p}\}) \mathrm{I}(\{\vec{a}\})=\sum_{\{\vec{f}\}} \sum_{\{i, j\}} \sum_{k \neq i, j} \int \mathrm{d} \Phi_{R}(\{\vec{p}\}) \mathrm{S}_{i j, k}(\{\vec{a}\}) .
$$

Identifying $\mathrm{D}_{i j, k}$ with $\mathrm{S}_{i j, k}$, the term with real-emission kinematics in eq. (2.26) can then be decomposed according to eq. (2.8), resulting in

$$
\sum_{\{\vec{f}\}} \mathrm{d} \Phi_{R}(\{\vec{p}\}) \sum_{\{i, j\}} \sum_{k \neq i, j}\left[\mathrm{R}_{i j, k}(\{\vec{a}\}) O(\{\vec{p}\})-\mathrm{S}_{i j, k}(\{\vec{a}\}) O\left(b_{i j, k}(\{\vec{p}\})\right)\right] .
$$

In the Powheg method, this term is approximated as

$$
\begin{aligned}
& \sum_{\{\vec{f}\}} \mathrm{d} \Phi_{R}(\{\vec{p}\}) \sum_{\{i, j\}} \sum_{k \neq i, j}\left[\mathrm{R}_{i j, k}(\{\vec{a}\})-\mathrm{S}_{i j, k}(\{\vec{a}\})\right] O\left(b_{i j, k}(\{\vec{p}\})\right) \\
& \quad=\sum_{\{\vec{f}\}} \mathrm{d} \Phi_{B}(\{\vec{p}\}) \sum_{\{\widetilde{\imath}, \tilde{k}\}} \sum_{f_{i}=q, g} \mathrm{~d} \Phi_{R \mid B}^{i j, k}\left[\mathrm{R}_{i j, k}\left(r_{\widetilde{\imath}, \tilde{k}}(\{\vec{a}\})\right)-\mathrm{S}_{i j, k}\left(r_{\widetilde{\imath}, \tilde{k}}(\{\vec{a}\})\right)\right] O(\{\vec{p}\}) .
\end{aligned}
$$


This allows the recombination of all contributions to the NLO cross section. Employing eq. (2.24), therefore

$$
\begin{aligned}
\overline{\mathrm{B}}(\{\vec{a}\})=\mathrm{B}(\{\vec{a}\})+\tilde{\mathrm{V}}(\{\vec{a}\})+\mathrm{I}(\{\vec{a}\}) & \\
& +\sum_{\{\widetilde{\imath}, \tilde{k}\}} \sum_{f_{i}=q, g} \int \mathrm{d} \Phi_{R \mid B}^{i j, k}\left[\mathrm{R}_{i j, k}\left(r_{\widetilde{\imath}, \tilde{k}}(\{\vec{a}\})\right)-\mathrm{S}_{i j, k}\left(r_{\widetilde{\imath}, \tilde{k}}(\{\vec{a}\})\right)\right] .
\end{aligned}
$$

Note that the difference with respect to an exact result lies in the phase-space dependence of the real-emission contribution to $\langle O\rangle$. To restore NLO accuracy, the following correction term must be added

$$
\sum_{\{\vec{f}\}} \mathrm{d} \Phi_{B}(\{\vec{p}\}) \sum_{\{\widetilde{\imath}, \tilde{k}\}} \sum_{f_{i}=q, g} \int \mathrm{d} \Phi_{R \mid B}^{i j, k} \mathrm{R}_{i j, k}\left(r_{\widetilde{\imath \jmath}, \tilde{k}}(\{\vec{a}\})\right)\left[O\left(r_{\widetilde{\imath \jmath}, \tilde{k}}(\{\vec{p}\})\right)-O(\{\vec{p}\})\right] .
$$

In the next section it will be shown that the combination of $\overline{\mathrm{B}}$ with a matrix-element corrected parton shower recovers exactly this correction.

\subsection{The Powheg method and its accuracy}

The key point of the Powheg method is, to supplement Monte Carlo event samples from matrix-element corrected parton showers with a next-to-leading order weight to arrive at full NLO accuracy. This is achieved by combining the two methods discussed in sections 2.3 and 2.4. To obtain the $\mathcal{O}\left(\alpha_{s}\right)$ approximation to the cross section in the PowHeg method, the parton-shower expression of the real-emission probability is combined with the approximated initial cross section, $\mathrm{d} \sigma_{\bar{B}}$. This yields the following master formula for the value of an infrared and collinear safe observable, $O$,

$$
\begin{aligned}
\langle O\rangle^{(\mathrm{POWHEG})=} \sum_{\{\vec{f}\}} & \int \mathrm{d} \Phi_{B}(\{\vec{p}\}) \overline{\mathrm{B}}(\{\vec{a}\})\left[\Delta^{(\mathrm{ME})}\left(t_{0}, \mu^{2} ;\{\vec{a}\}\right) O(\{\vec{p}\})\right. \\
& +\sum_{\{\widetilde{\jmath}, \tilde{k}\}} \sum_{f_{i}=q, g} \frac{1}{16 \pi^{2}} \int_{t_{0}}^{\mu^{2}} \mathrm{~d} t \int_{z_{\min }}^{z_{\max }} \mathrm{d} z \int_{0}^{2 \pi} \frac{\mathrm{d} \phi}{2 \pi} J_{i j, k}(t, z, \phi) \\
& \left.\times \frac{1}{S_{i j}} \frac{S\left(r_{\widetilde{\imath}, \tilde{k}}(\{\vec{f}\})\right)}{S(\{\vec{f}\})} \frac{\mathrm{R}_{i j, k}\left(r_{\widetilde{\imath}, \tilde{k}}(\{\vec{a}\})\right)}{\mathrm{B}(\{\vec{a}\})} \Delta^{(\mathrm{ME})}\left(t, \mu^{2} ;\{\vec{a}\}\right) O\left(r_{\widetilde{\imath}, \tilde{k}}(\{\vec{p}\})\right)\right],
\end{aligned}
$$

where obvious arguments of the parton maps $r_{\widetilde{\imath}, \tilde{k}}$ have been suppressed. Clearly, if the observable $\mathcal{O}$ on the right hand side of eq. (2.32) becomes one, the quantity computed is the total cross section, as for the cases discussed in sections 2.2 and 2.3. This particular case, however, is insensitive to the details of the radiation pattern. Equation (2.32) can be 
rearranged as $[1,2]$

$$
\begin{aligned}
& \langle O\rangle^{(\text {POWheG })}=\sum_{\{\vec{f}\}} \int \mathrm{d} \Phi_{B}(\{\vec{p}\}) \overline{\mathrm{B}}(\{\vec{a}\})[O(\{\vec{p}\}) \\
& +\sum_{\{\widetilde{\imath}, \tilde{k}\}} \sum_{f_{i}=q, g} \frac{1}{16 \pi^{2}} \int_{t_{0}}^{\mu^{2}} \mathrm{~d} t \int_{z_{\min }}^{z_{\max }} \mathrm{d} z \int_{0}^{2 \pi} \frac{\mathrm{d} \phi}{2 \pi} J_{i j, k}(t, z, \phi) \\
& \quad \times \frac{1}{S_{i j}} \frac{S\left(r_{\widetilde{\imath}, \tilde{k}}(\{\vec{f}\})\right)}{S(\{\vec{f}\})} \frac{\mathrm{R}_{i j, k}\left(r_{\widetilde{\imath}, \tilde{k}}(\{\vec{a}\})\right)}{\mathrm{B}(\{\vec{a}\})} \Delta^{(\mathrm{ME})}\left(t, \mu^{2} ;\{\vec{a}\}\right)\left[O\left(r_{\widetilde{\imath}, \tilde{k}}(\{\vec{p}\})\right)-O(\{\vec{p}\})\right],
\end{aligned}
$$

Two special cases should now be considered $[1,2]$

- The infrared limit $(t \rightarrow 0)$

In this case, only the first term in eq. (2.33) contributes, as any infrared safe observable maps the real-emission kinematics for collinear (soft) emissions to the kinematics of the (any) underlying Born configuration

$$
O\left(r_{\widetilde{\imath \jmath}, \tilde{k}}(\{\vec{p}\})\right) \stackrel{t \rightarrow 0}{\rightarrow} O(\{\vec{p}\})
$$

The contribution to $\langle O\rangle^{\text {(POWHEG) }}$ from this phase-space region is therefore correct to $\mathcal{O}\left(\alpha_{s}\right)$.

- Hard emissions $\left(t \rightarrow \mu^{2}\right)$

In this case $\Delta^{(\mathrm{ME})} \rightarrow 1$ and to $\mathcal{O}\left(\alpha_{s}\right)$ one can replace $\overline{\mathrm{B}} \rightarrow \mathrm{B}$. Thus, the second term in eq. (2.33) becomes

$$
\begin{aligned}
& \sum_{\{\vec{f}\}} \int \mathrm{d} \Phi_{B}(\{\vec{p}\}) \sum_{\{\widetilde{\imath}, \tilde{k}\}} \sum_{f_{i}=q, g} \frac{1}{16 \pi^{2}} \int^{\mu^{2}} \mathrm{~d} t \int \mathrm{d} z \int \frac{\mathrm{d} \phi}{2 \pi} J_{i j, k}(t, z, \phi) \\
& \quad \times \frac{S\left(r_{\widetilde{\imath \jmath}, \tilde{k}}(\{\vec{f}\})\right)}{S(\{\vec{f}\}) S_{i j}} \mathrm{R}_{i j, k}\left(r_{\widetilde{\imath}, \tilde{k}}(\{\vec{a}\})\right)\left[O\left(r_{\widetilde{\imath}, \tilde{k}}(\{\vec{p}\})\right)-O(\{\vec{p}\})\right] .
\end{aligned}
$$

Comparing this result with the correction term, eq. (2.31), reveals that both expressions differ by the factor $S(\{\vec{f}\}) S_{i j} / S\left(r_{\widetilde{\imath}, \tilde{k}}(\{\vec{f}\})\right)$, which arises solely due to the way the real-emission phase space is populated by the parton shower (cf. section 2.2).

Therefore, the contribution to $\langle O\rangle^{(\mathrm{POWHEG})}$ from this phase-space region is correct to $\mathcal{O}\left(\alpha_{s}\right)$.

In the phase-space regions "between" these limits, the POWHEG method interpolates smoothly between the two above results.

\section{Realisation of the Powheg method in the SHerpa Monte Carlo}

Sherpa is a multi-purpose Monte-Carlo event generator for collider experiments $[3,4]$. The goal of this project is a complete simulation of all aspects of the collision. Despite 
being focused on developments improving the treatment of perturbative QCD, over the past years significant improvements have been achieved regarding the description of soft QCD and QED dynamics, like the process of hadronisation, the decays of the produced hadrons, and the implementation of QED radiation in these decays [74]. One of the traditional key features of the SHERPA program, however, is a consistent merging of multi-jet matrix elements at tree-level with the subsequent parton shower in the spirit of $[10,11]$. An improvement of this method and its consistent implementation have been presented in [17] and extended to include hard QED radiation [73]. To this end, SHERPA uses its two internal tree-level matrix element generators AMEGIC $++[75]$ and ComIx [76], which are capable of calculating cross sections for processes in the Standard Model (AMEGIC ++ and Comix) and beyond (AMEGIC ++ ), involving final states with high multiplicities. Soft and collinear parton radiation is generated in SHERPA by means of a parton shower based on CataniSeymour dipole factorisation [67]. The program also allows to steer external modules for the computation of virtual corrections using a standardised interface [77]. The corresponding real corrections and subtraction terms in the Catani-Seymour formalism [48, 49] are then provided automatically by AmEGiC $++[78]$.

SHERPA is therefore perfectly suited to implement the POWHEG method as all prerequisites outlined in section 2 are found within a single, coherent framework. In this section the basic features of the corresponding modules of the generator are reviewed, as far as they are important in the context of this work. A complete overview of SHERPA can be found in $[3,4]$.

\subsection{Matrix elements and subtraction terms}

For this study, the matrix-element generator AmEGIC++ [75] is employed. It is based on the construction of Feynman diagrams, which are evaluated using the helicity methods introduced in $[79,80]$. For the computation of NLO cross sections in QCD-associated processes, AMEGIC ++ provides the fully automated generation of dipole subtraction terms [78], implementing the Catani-Seymour formalism [48, 49]. As outlined in section 2.4, such a subtraction procedure is a necessary ingredient to be able and compute NLO QCD cross sections with Monte-Carlo methods.

In the Catani-Seymour method, the soft and collinear singularities of the real-emission amplitude squared, $\mathcal{R}(\{\vec{a}\})$, are removed by a local subtraction term (cf. eq. (2.26))

$$
\begin{aligned}
\mathcal{S}(\{\vec{a}\}) & =\sum_{\{i, j\}} \sum_{k \neq i, j} \mathcal{S}_{i j, k}(\{\vec{a}\}) \\
& =\sum_{i, j} \sum_{k \neq i, j} \tilde{\mathcal{D}}_{i j, k}(\{\vec{a}\})+\sum_{i, j} \sum_{a} \tilde{\mathcal{D}}_{i j}^{a}(\{\vec{a}\})+\sum_{a, j} \sum_{k \neq j} \tilde{\mathcal{D}}_{k}^{a j}(\{\vec{a}\})+\sum_{a, j} \sum_{b \neq a} \tilde{\mathcal{D}}^{a j, b}(\{\vec{a}\}) .
\end{aligned}
$$

On the first line, the notation of section 2.4 is adopted, while on the second line the definitions of $[48,49]$ are restored by defining $i, j, k>2, a, b=1,2$ and requiring all indices to be mutually distinct. Along those lines, $\tilde{\mathcal{D}}_{i j, k}, \tilde{\mathcal{D}}_{i j}^{a}, \tilde{\mathcal{D}}_{k}^{a j}$ and $\tilde{\mathcal{D}}^{a j, b}$ are the four types of Catani-Seymour dipole terms, as depicted in figure 1, for final-state splittings with final-state spectators, final-state splittings with initial-state spectators, initial-state 
(1) $\mathrm{FF}$

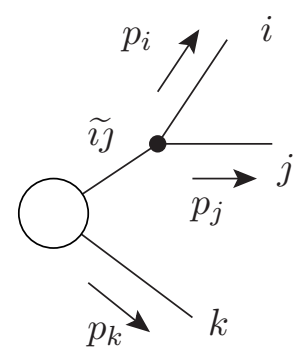

(3) IF

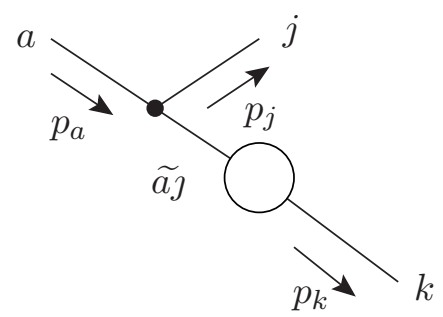

(2) FI

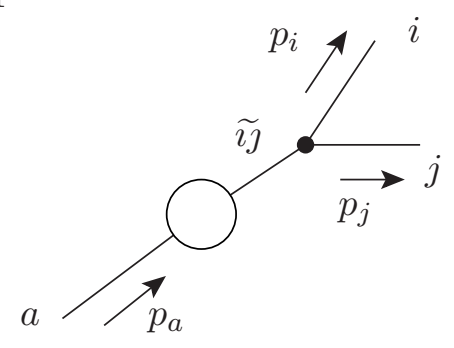

(4) II

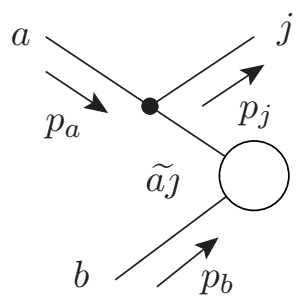

Figure 1. Effective diagram for the splitting of (1) a final-state parton connected to a final-state spectator, (2) a final-state parton connected to an initial-state spectator, (3) an initial-state parton connected to a final-state spectator and (4) an initial-state parton connected to an initial-state spectator in the standard Catani-Seymour notation. The blob denotes the colour correlated leading order matrix element, and the incoming and outgoing lines label the initial-state and final-state partons participating in the splitting.

splittings with final-state spectators and initial-state splittings with initial-state spectators, respectively. This implies that for final-state splittings, i.e. $i, j>2$,

$$
\mathcal{S}_{i j, k}=\mathcal{S}_{j i, k}=\frac{1}{2} \tilde{\mathcal{D}}_{i j, k} \quad \text { and } \quad \mathcal{S}_{i j, a}=\mathcal{S}_{j i, a}=\frac{1}{2} \tilde{\mathcal{D}}_{i j}^{a},
$$

while for initial-state splittings

$$
\mathcal{S}_{a j, k}=\tilde{\mathcal{D}}_{k}^{a j} \quad \text { and } \quad \mathcal{S}_{a j, b}=\tilde{\mathcal{D}}^{a j, b} .
$$

Due to its analytic integrability over the extra emission phase space $\mathrm{d} \Phi_{R \mid B}^{i j, k}$ in $D=4-2 \epsilon$ dimensions, the subtraction term $\mathcal{S}$, in its integrated form $\mathcal{I}$ of eq. (2.27), as well as the collinear counter-terms can be added back to the virtual contributions to cancel their poles in $\epsilon$,

$$
\{\epsilon[\tilde{\mathcal{V}}(\{\vec{a}\})+\mathcal{I}(\{\vec{a}\})]\}_{\epsilon=0}=\{\epsilon[\mathcal{V}(\{\vec{a}\})+\mathcal{I}(\{\vec{a}\})+\mathcal{C}(\{\vec{a}\})]\}_{\epsilon=0}=0
$$

wherein $\mathcal{V}$ is the one-loop matrix element convoluted with the Born amplitude and $\mathcal{C}$ is the collinear counter-term.

The implementation in SHERPA's matrix element generator AMEGIC++, expanding upon its tree-level capabilities to generate $\mathcal{B}$ and $\mathcal{R}$, is able to generate both the subtraction 


\begin{tabular}{|c|c|c|}
\hline Type & $\alpha$ & $z$ \\
\hline FF & $y_{i j, k}$ & $\tilde{z}_{i}$ \\
FI & $1-x_{i j, k}$ & $\tilde{z}_{i}$ \\
\hline
\end{tabular}

\begin{tabular}{|c|c|c|}
\hline Type & $\alpha$ & $z$ \\
\hline IF & $u_{j}$ & $x_{j k, a}$ \\
II & $\tilde{v}_{j}$ & $x_{j, a b}$ \\
\hline
\end{tabular}

Table 1. Definition of integration variables in eq. (3.5) for the various dipole configurations of figure 1.

terms $\mathcal{S}$ and their integrated counterparts $\mathcal{I}$ as well as the collinear counter-term $\mathcal{C}$ in an automated fashion. The virtual contributions $\mathcal{V}$, however, are obtained from dedicated external codes interfaced using the Binoth-Les Houches Accord [77]. Having all this at hand, the assembly of the $\overline{\mathrm{B}}$-function of eq. (2.30), integrable in $D=4$ dimensions, is feasible in an automated way. This involves integrating over the real-emission subspace of the phase space of the NLO real correction to the Born process, cf. eq. (2.30), and adding the result to the terms with Born-level kinematics.

In SHERPA, this integration is performed in a Monte-Carlo fashion, by selecting a single point in the real-emission phase space. This technique potentially generates negative weights. In the standard POWHEG method, the emergence of such negative weights is suppressed by either performing the integration analytically or by sampling over sufficiently many real-emission phase-space points. Tests to decide which method is better for practical applications are beyond the scope of this publication and will be addressed in a future work; here it should suffice to state that, of course, sampling over more than one phase space point is neither a conceptual problem nor a practical obstacle. Also, for all processes under study in this publication, no problems have been encountered by the possibility of having negative weighted events. Loosely speaking, the problem can in no case be more severe than the possibility of having negative weights in a standard NLO calculation. Therefore, the only remaining issue is to construct an integration method, which, starting from a given Born configuration, is able to fill the real-emission phase space in an efficient manner. Having an implementation of the Catani-Seymour subtraction method at hand, the construction of an integrator for the real-emission subspace based on CS-subtraction terms is rendered a straightforward exercise. The actual integration can be decomposed into three one-dimensional integrals (cf. eq. (2.14)) [48, 49]

$$
\mathrm{d} \Phi_{R \mid B}^{i j, k}=\frac{2 p_{\widetilde{\imath \jmath}} p_{\tilde{k}}}{16 \pi^{2}} \mathrm{~d} \alpha \mathrm{d} z \frac{\mathrm{d} \phi}{2 \pi} \tilde{J}_{i j, k}(\alpha, z, \phi)
$$

with the two integration variables $\alpha$ and $z$ given in table 1, cf. [78]. The azimuthal angle, $\phi$, is common to all configurations.

Several different integration channels, each based on a separate CS dipole, can be combined to yield a multi-channel integrator [81] for the real-emission phase space. The apriori weights in the multi-channel can be employed to better adapt to the emission pattern of the process under consideration. Additionally, every one-dimensional integrator can be individually improved using the VEGAS algorithm [82, 83]. 


\begin{tabular}{|c|c|c|c|}
\hline Type & $z_{i, j k}$ & $\xi_{i, j k}$ & $\tilde{y}_{i j, k}$ \\
\hline FF & $\tilde{z}_{i}$ & $\tilde{z}_{i}$ & $y_{i j, k}$ \\
\hline FI & $\tilde{z}_{i}$ & $-\tilde{z}_{i}$ & $\frac{1-x_{i j, a}}{x_{i j, a}}$ \\
\hline
\end{tabular}

\begin{tabular}{|c|c|c|c|}
\hline Type & $z_{j, a k}$ & $\xi_{j, a k}$ & $\tilde{y}_{j a, k}$ \\
\hline IF & $x_{j k, a}$ & $\left|1-\frac{1-u_{j}}{x_{j k, a}-u_{j}}\right|$ & $\frac{u_{j}}{x_{j k, a}}$ \\
\hline II & $x_{j, a b}$ & $1-\frac{1}{x_{j, a b}+\tilde{v}_{j}}$ & $\frac{\tilde{v}_{j}}{x_{j, a b}}$ \\
\hline
\end{tabular}

Table 2. Mapping of variables for eqs. (3.6), (3.7) and (3.15). Note that the definitions for massless partons in $[48,49]$ are employed.

\subsection{The parton shower}

SHERPA implements a parton shower based on Catani-Seymour (CS) dipole factorisation, which will be denoted by Css [67]. The model was originally proposed in [84, 85] and worked out and implemented in parallel in $[67,68]$. It relies on the factorisation of realemission matrix elements in the CS subtraction framework [48, 49]. Since the original algorithm has been improved in several ways, which have not yet been compiled in a single reference, the parton-shower model and its basic features are briefly reviewed here.

\subsubsection{Ordering parameters and splitting functions}

Consider the process depicted in figure 1, where a parton $\widetilde{\imath \jmath}$, accompanied by a spectator parton $\tilde{k}$, splits into partons $i$ and $j$, with the recoil absorbed by the spectator $k$. Conveniently the combined momenta are identified as $p_{i j}=p_{i}+p_{j}$ and $Q=p_{i j}+p_{k}$. A Lorentz-invariant transverse momentum, $\tilde{\mathrm{k}}_{T}^{2}$, which acts as ordering parameter in the parton shower algorithm, can now be defined as

$$
\tilde{\mathrm{k}}_{T}^{(\mathrm{FS}) 2}=\left|Q^{2}-m_{i}^{2}-m_{j}^{2}-m_{k}^{2}\right| \tilde{y}_{i j, k} z_{i, j k}\left(1-z_{i, j k}\right)-\left(1-z_{i, j k}\right)^{2} m_{i}^{2}-z_{i, j k}^{2} m_{j}^{2},
$$

where $m_{i}, m_{j}$ and $m_{k}$ are the parton masses. The above relation holds, independent of whether the spectator parton is a final- or initial-state particle for all final-state splittings, while for initial-state splittings $a \rightarrow \widetilde{a \jmath} j$, again independent of the type of spectator, the ordering parameter is given by

$$
\tilde{\mathrm{k}}_{T}^{(\mathrm{IS}) 2}=\left|Q^{2}-m_{j}^{2}-m_{a}^{2}-m_{k}^{2}\right| \tilde{y}_{j a, k}\left(1-z_{j, a k}\right)-m_{j}^{2}-\left(1-z_{j, a k}\right)^{2} m_{a}^{2} .
$$

The precise definition of $\tilde{y}$ and the splitting variables $z$ for the various dipole types are listed in table 2 .

Sudakov form factors for all branching types, taking into account finite masses of finalstate partons and strictly relying on the Lorentz-invariant variables $z$ and $\tilde{\mathrm{k}}_{T}^{2}$, have been derived. The corresponding evolution kernels, as defined in eq. (2.11), read

$$
\mathcal{K}_{i j, k}\left(\tilde{\mathrm{k}}_{T}^{2}, z\right)=\frac{1}{\bar{S}_{i j}} \frac{1}{\mathcal{N}_{\text {spec }}}\left\langle\mathrm{V}_{i j, k}\left(\tilde{\mathrm{k}}_{T}^{2}, z\right)\right\rangle
$$

where $\mathcal{N}_{\text {spec }}$ is the number of spectator partons of the off-shell particle $a_{\widetilde{\imath} \jmath}$ in the large $N_{C}$ limit. The spin-averaged dipole functions $\langle\mathrm{V}\rangle$, taken in four dimensions, depend on the 
type of emitter and spectator parton and are listed in [67]. Their infrared singularities are regularised through the parton shower cutoff, $t_{0}$, typically of the order of $1 \mathrm{GeV}^{2}$. The denominator factor $\bar{S}_{i j}$ avoids double-counting identical final states in final-state evolution and is given by

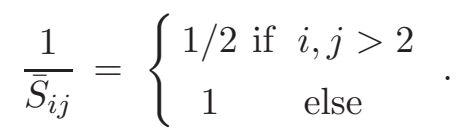

A potential shortcoming of the original approach in [67] is that certain dipole functions connecting the initial and final state may acquire negative values in some non-singular regions of the phase space. This prohibits their naive interpretation in terms of splitting probabilities and leaves the corresponding parts of the phase space unpopulated. The problem was solved recently by altering the finite parts of the affected splitting functions such that they reproduce corresponding full matrix elements [86].

\subsubsection{Splitting kinematics}

All branchings in the Css formalism implement exact four-momentum conservation and the particles are kept on their mass-shell before and after every evolution step. The phase-space maps from $n$ - to $n+1$-particle final states are exact and cover the whole phase space. They are, however, not unambiguous. One of the most prominent criticisms of this particular parton-shower model is the "unphysical" recoil strategy originally employed in configurations with initial-state splitter and final-state spectator. This problem was addressed in [72] for the case of massless partons and has been implemented for prompt photon production and extended to the fully massive case in [73]. The latter publication defined a general algorithm for constructing the emission kinematics, independent of the type of splitter and spectator parton, which is summarised as follows:

1. Determine the new momentum of the spectator parton as $[48,49]$

$$
p_{k}=\left(\tilde{p}_{k}-\frac{Q^{2}+m_{k}^{2}-m_{i j}^{2}}{2 Q^{2}} Q\right) \sqrt{\frac{\lambda\left(Q^{2}, s_{i j}, m_{k}^{2}\right)}{\lambda\left(Q^{2}, m_{i j}^{2}, m_{k}^{2}\right)}}+\frac{Q^{2}+m_{k}^{2}-s_{i j}}{2 Q^{2}} Q,
$$

with $\lambda$ denoting the Källen function $\lambda(a, b, c)=(a-b-c)^{2}-4 b c$ and

$$
s_{i j}=\tilde{y}_{i j, k}\left(Q^{2}-m_{k}^{2}\right)+\left(1-\tilde{y}_{i j, k}\right)\left(m_{i}^{2}+m_{j}^{2}\right) .
$$

2. Find the light-like helper vectors $l$ and $n$ as

$$
l=\frac{p_{i j}-\alpha_{i j} p_{k}}{1-\alpha_{i j} \alpha_{k}}, \quad n=\frac{p_{k}-\alpha_{k} p_{i j}}{1-\alpha_{i j} \alpha_{k}},
$$

where $\alpha_{i j}=s_{i j} / \gamma_{i j, k}, \alpha_{k}=m_{k}^{2} / \gamma_{i j, k}$ and

$$
\gamma_{i j, k}=2 l \cdot n=\frac{1}{2}\left[\left(Q^{2}-s_{i j}-m_{k}^{2}\right)+\operatorname{sgn}\left(Q^{2}-s_{i j}-m_{k}^{2}\right) \sqrt{\lambda\left(Q^{2}, s_{i j}, m_{k}^{2}\right)}\right] .
$$


3. Express the momenta $p_{i}$ and $p_{j}$ in terms of $l, n$ and a transverse component $k_{\perp}$ as

$$
p_{i}^{\mu}=\bar{z}_{i} l^{\mu}+\frac{m_{i}^{2}+\mathrm{k}_{\perp}^{2}}{\bar{z}_{i}} \frac{n^{\mu}}{2 l \cdot n}+k_{\perp}^{\mu}, \quad p_{j}^{\mu}=\left(1-\bar{z}_{i}\right) l^{\mu}+\frac{m_{j}^{2}+\mathrm{k}_{\perp}^{2}}{1-\bar{z}_{i}} \frac{n^{\mu}}{2 l \cdot n}-k_{\perp}^{\mu},
$$

The parameters $\bar{z}_{i}$ and $\mathrm{k}_{\perp}^{2}$ of this decomposition are given by

$$
\begin{aligned}
\bar{z}_{i} & =\frac{Q^{2}-s_{i j}-m_{k}^{2}}{\sqrt{\lambda\left(Q^{2}, s_{i j}, m_{k}^{2}\right)}}\left[\xi_{i, j k}-\frac{m_{k}^{2}}{\left|\gamma_{i j, k}\right|}\left(\frac{\tilde{y}_{i j, k}}{1-\tilde{y}_{i j, k}}+\frac{2 m_{i}^{2}}{Q^{2}-s_{i j}-m_{k}^{2}}\right)\right], \\
\mathrm{k}_{\perp}^{2} & =\left(Q^{2}-m_{i}^{2}-m_{j}^{2}-m_{k}^{2}\right) \tilde{y}_{i j, k} \bar{z}_{i}\left(1-\bar{z}_{i}\right)-\left(1-\bar{z}_{i}\right)^{2} m_{i}^{2}-\bar{z}_{i}^{2} m_{j}^{2},
\end{aligned}
$$

where $\xi_{i, j k}$ is defined in table 2. Equations (3.10) and (3.15) are valid for all dipole configurations, i.e. initial and final-state branchings with the recoil partner being either in the initial or in the final state.

To generate an emission using the matrix-element correction technique presented in section 2.3, it is important to be able and access matrix-element information during the parton-shower evolution, such that eq. (2.21) can be implemented in a process-independent manner. SHERPA provides an interface between its tree-level matrix-element generators and its parton shower, which allows for all the necessary interactions. Together with an implementation of the phase-space maps $b_{i j, k}(\{\vec{a}\})$, that correspond to the inverse of the above splitting kinematics, eq. (2.21) can then be realised easily.

\subsection{Automatic identification of Born zeros}

It was noted in [32] that eq. (2.21) can develop spurious singularities as the matrix element of the underlying Born process may be zero, while the real-emission matrix element is not. Such configurations do not exponentiate, as $\mathcal{R}$ is not singular when $\mathcal{B} \rightarrow 0$. This fact can be employed to formulate a general solution to the problem [32]. One can split $\mathcal{R}$ into two parts, a singular one, $\mathcal{R}^{(\mathrm{s})}$, and a regular one, $\mathcal{R}^{(\mathrm{r})}$.

$$
\mathcal{R}^{(\mathrm{s})}=\mathcal{R} \frac{Z}{Z+H}, \quad \text { and } \quad \mathcal{R}^{(\mathrm{r})}=\mathcal{R} \frac{H}{Z+H},
$$

where

$$
Z=\frac{\mathcal{B}}{\mathcal{B}_{\max }}, \quad \text { and } \quad H=\kappa_{\text {res }}^{2} \frac{t}{t_{\max }} .
$$

Note that $\mathcal{B}_{\max }$ can be determined during the integration of the seed cross section, while $t_{\text {max }}$ is given as a universal function of the hadronic centre-of-mass energy, depending only on the definition of $t$ in the parton shower model. The resolution factor $\kappa_{\text {res }}$ then determines the relative splitting between $\mathcal{R}^{(\mathrm{s})}$ and $\mathcal{R}^{(\mathrm{r})}$ : the larger $\kappa_{\text {res }}$, the larger the fraction $\mathcal{R}^{(\mathrm{r})}$ of $\mathcal{R}$.

The necessity of such a splitting of the real emission matrix element can be determined on an event-by-event basis by comparing the correction factor of eq. (2.20), $w_{i j, k}$, to a predefined threshold $w_{i j, k}^{\text {th }}$. Thus, regular non-exponentiated $\mathcal{R}^{(\mathrm{r})}$ events are only produced if $w_{i j, k}>w_{i j, k}^{\mathrm{th}}$. Such a treatment ensures that both the exponentiation of the real-emission matrix element is as inclusive as possible and the parton-shower correction factor does not get too large, rendering event generation too inefficient. 


\section{Results}

This section collects results obtained with the implementation of the POWHEG algorithm in the SHERPA event generator. We exemplify the performance in a variety of processes which are listed in section 4.1 with their respective cuts and relevant settings. Where applicable, the CTEQ6.6 [87] parton distribution functions have been employed and the strong coupling has been defined accordingly as $\alpha_{s}\left(m_{Z}\right)=0.118$ with NLO running for both the matrix elements and the parton shower. In section 4.2 the internal consistency of the implementation is checked by performing scale variations, cross section comparisons with ordinary NLO calculations, and variations of internal parameters of PowHEG. Comparisons of results from the new implementation with predictions from tree-level matrix-element parton-shower merging $(\mathrm{ME}+\mathrm{PS})$ are presented in section 4.3. Finally, comparisons with experimental data are made in section 4.4. For DIS analyses we used the HZTool framework [88] while all other analyses have been performed using Rivet and are documented in the corresponding manual [89].

\subsection{Process listing}

\subsubsection{Jet production in $e^{+} e^{-}$collisions}

The annihilation of $e^{+} e^{-}$into hadrons is studied at LEP Run 1 energies, $E_{\mathrm{CMS}}=$ 91.25 GeV. This setup allows to validate the algorithms of section 2 in pure final-state QCD evolution, which is the simplest testing ground. The parton shower cut-off scale has been set to $k_{T \text {, min }}^{2}=1.6 \mathrm{GeV}^{2}$. Even though the improvements in this paper are purely related to perturbative physics, the results are presented after hadronisation with the Lund model $[5,90,91]$ to make comparison to experimental results more meaningful. The ME+PS samples have been generated with up to one additional jet in the matrix elements and the phase space slicing parameter was set to $\log \left(y_{\text {cut }}\right)=-2.25$. For the virtual matrix elements, the code provided by the BlackHat collaboration [50-52] was used.

\subsubsection{Deep-inelastic lepton-nucleon scattering}

Hadronic final states in deep-inelastic lepton-nucleon scattering (DIS) are studied at HERA Run 1 energies, $E_{\mathrm{CMS}}=300 \mathrm{GeV}$. Just like $e^{+} e^{-}$-annihilation into hadrons, this process boasts a wealth of precise experimental data. From the theoretical perspective, it is invaluable, as it allows to test QCD factorisation in an extremely clean environment. The associated scale, given by the virtuality of the exchanged $\gamma^{*} / Z$-boson is not fixed, but potentially varies by orders of magnitude, which allows to test perturbative QCD predictions in various kinematic limits. Our results are presented at the parton level only, as hadronisation corrections have little effect on the observables and the focus lies on the potential improvements in the perturbative part of the simulation. The Monte-Carlo settings correspond to those in [86]. ME+PS samples have been generated with up to one additional jet in the matrix element and the phase space slicing parameters were set to $\bar{Q}_{\text {cut }}=5$ and $S_{\text {DIS }}=0.6$ (cf. [86]). Virtual matrix elements were provided by BlackHat [50-52]. 


\subsubsection{Drell-Yan lepton pair production}

We investigate Drell-Yan lepton pair production at Tevatron Run 2 energies, simulating $p \bar{p}$ collisions at $E_{\mathrm{CMS}}=1.96 \mathrm{TeV}$. A cut on the invariant mass of the lepton pair of $66<$ $m_{\ell \ell} / \mathrm{GeV}<116$ is applied at the matrix-element level. For the ME+PS samples matrix elements with up to one additional jet were generated and a phase-space slicing cut of $Q_{\text {cut }}=20 \mathrm{GeV}$ was applied. Virtual matrix elements were provided by BlackHat [50$52]$. The factorisation and renormalisation scales for the NLO matrix element were chosen as $\mu_{R}^{2}=\mu_{F}^{2}=m_{\perp, \ell \ell}^{2}$. In all tree-level matrix elements SHERPA's default scale choice was employed: The matrix element is clustered onto a core $2 \rightarrow 2$ configuration using a $k_{T}$-type algorithm with recombination into on-shell particles. Scales are defined as the lowest invariant mass or negative virtuality in the core process. Hadronisation and multiple parton interactions have been disabled to allow for a study at the parton-shower level. The $Z \rightarrow \ell \ell$ decay is corrected for QED next-to-leading order and soft-resummation effects in the Yennie-Frautschi-Suura (YFS) approach [74].

The three reactions listed in section 4.1.1-4.1.3 essentially amount to one and the same process at the parton level, as they only differ by crossing of initial- and final-state legs. Their combination allows to validate the implementation of the matrix-element corrections in section 2.3 for all possible dipole configurations with quark splitters.

\subsubsection{W boson production}

Production of $W$ bosons is presented in $p \bar{p}$ collisions at $E_{\mathrm{CMS}}=1.8 \mathrm{TeV}$. Although in principle similar to the Drell-Yan case, this process is of special interest to validate the automatic decomposition of the real-emission term into singular and non-singular pieces, as outlined in section 3.3. If not stated otherwise, the parameters for this decomposition are set to $\kappa_{\text {res }}=4$ and $w_{i j, k}^{\text {th }}=100$. A cut on the invariant mass of the lepton-neutrino pair of $m_{\ell \nu}>10 \mathrm{GeV}$ was applied at the matrix-element level. For the ME+PS samples matrix elements with up to one additional jet were used and a phase space slicing cut of $Q_{\text {cut }}=20 \mathrm{GeV}$ was applied. Virtual matrix elements were provided by BlackHat [50-52]. The factorisation and renormalisation scales for the NLO matrix element were chosen as $\mu_{R}^{2}=\mu_{F}^{2}=m_{\perp, \ell \nu}^{2}$. In all tree-level matrix elements SHERPA's default scale choice was employed, cf. section 4.1.3. Hadronisation and multiple parton interactions have been disabled. The $W \rightarrow \ell \nu$ decay is corrected for QED next-to-leading order and soft-resummation effects in the YFS approach [74].

\subsubsection{Higgs boson production through gluon-gluon fusion}

The production of Higgs bosons through gluon-gluon fusion is simulated for proton-proton collisions at $E_{\mathrm{CMS}}=14 \mathrm{TeV}$. The coupling to gluons is mediated by a top-quark loop and modeled through an effective Lagrangian [92, 93]. Again, this process is technically very similar to the Drell-Yan case, but it also allows to validate matrix-element corrections to the remaining initial-state splitting functions. Next-to-leading order corrections are rather large at nominal LHC energies, with a ratio of $K \approx 2$ between the NLO and the LO result for the total cross section. This fact has spurred tremendous efforts to perform 
fully differential calculations at NNLO [94-96] and several predictions have been presented which merged such fixed-order results with resummation at next-to-next-to-leading logarithmic accuracy [97, 98], as the process is expected to have high phenomenological relevance at LHC energies. However, this publication centres on the behaviour of the theory at NLO only, as a prediction beyond this level of accuracy is clearly not within the capabilities of the Powheg method.

We consider a decay of the Higgs boson into the $\tau^{+} \tau^{-}$final state. A cut on the invariant mass of the $\tau$ pair of $115<m_{\tau \tau} / \mathrm{GeV}<125$ was applied at the matrix-element level. For the ME+PS merged samples matrix elements with up to one additional jet were used and a phase-space slicing cut of $Q_{\text {cut }}=20 \mathrm{GeV}$ was applied. The virtual matrix elements have been implemented according to [37]. The factorisation and renormalisation scales for the NLO matrix element were chosen as $\mu_{R}^{2}=\mu_{F}^{2}=m_{\perp, \tau \tau}^{2}$. In all tree-level matrix elements SHERPA's default scale choice was employed, cf. section 4.1.3. Hadronisation and multiple parton interactions have been disabled. The $h \rightarrow \tau \tau$ decay is corrected for QED soft-resummation and approximate next-to-leading order effects in the YFS approach [74].

\subsubsection{Z-pair production}

The production of pairs of $Z$ bosons is studied for proton-proton collisions at $E_{\mathrm{CMS}}=$ $14 \mathrm{TeV}$. This is an important background for the golden-plated Higgs-boson discovery mode at the LHC. Detailed studies of the decay properties of the $Z$ bosons and their correlations are known to allow for a determination of some properties of the Higgs boson, when found. Among these correlations are, e.g. the relative orientation of the decay planes of the bosons.

A cut on the invariant mass of each lepton pair of $66<m_{\ell \ell} / \mathrm{GeV}<116$ was applied at the matrix-element level. For the ME+PS samples matrix elements with up to one additional jet were used and a phase-space slicing cut of $Q_{\text {cut }}=20 \mathrm{GeV}$ was applied. Virtual matrix elements were provided by $\operatorname{McFM}[53,99,100]$. The factorisation and renormalisation scales were chosen as $\mu_{R}^{2}=\mu_{F}^{2}=m_{Z Z}^{2}$. Hadronisation and multiple parton interactions have been disabled to allow for a study at the parton shower level. Each $Z \rightarrow \ell \ell$ decay is corrected for QED next-to-leading order and soft-resummation effects in the YFS approach [74].

\subsection{7 $W^{+} W^{-}$-production}

$W^{+} W^{-}$-production is also studied for proton-proton collisions at $E_{\mathrm{CMS}}=14 \mathrm{TeV}$. It is worth noting that this process hitherto has not been treated in the POwHEG approach. Similar to the $Z$-pair production, it is an important background to the search channel for the Standard-Model Higgs boson, at masses around and above $130 \mathrm{GeV}$. Again, in order to suppress this background, distributions which depend on correlations of decay products of the $W$ 's in phase space are heavily used.

A cut on the invariant mass of each lepton-neutrino pair of $m_{\ell \nu}>10 \mathrm{GeV}$ was applied at the matrix-element level. For the ME+PS samples matrix elements with up to one additional jet were used and a phase-space slicing cut of $Q_{\text {cut }}=20 \mathrm{GeV}$ was applied. Virtual matrix elements were provided by MCFM [53, 99, 100]. The factorisation and renormalisation scales were chosen as $\mu_{R}^{2}=\mu_{F}^{2}=m_{W W}^{2}$. Hadronisation and multiple 


\begin{tabular}{|c|c|c|c|c|c|}
\hline \multicolumn{2}{|c|}{} & \multicolumn{2}{|c|}{$e^{+} e^{-} \rightarrow$ hadrons } & \multicolumn{2}{c|}{$e^{+} p \rightarrow e^{+}+j+X$} \\
\cline { 2 - 6 } \multicolumn{2}{|c|}{} & \multicolumn{2}{|c|}{$E_{\mathrm{cms}}=91.2 \mathrm{GeV}$} & \multicolumn{2}{c|}{$\begin{array}{c}E_{\mathrm{cms}}=300 \mathrm{GeV} \\
Q^{2}>150 \mathrm{GeV}^{2}\end{array}$} \\
\hline$\mu=\mu_{R}=\mu_{F}$ & Factor & PowHEG & NLO & PowHEG & NLO \\
\hline \multirow{3}{*}{$\sqrt{Q^{2}}$} & $1 / 2$ & $30179(18)$ & $30195(20)$ & $3906(9)$ & $3908(10)$ \\
\cline { 2 - 6 } & 1 & $29411(17)$ & $29416(18)$ & $4047(10)$ & $4050(11)$ \\
\cline { 2 - 6 } & 2 & $28680(16)$ & $28697(18)$ & $4180(10)$ & $4188(11)$ \\
\hline
\end{tabular}

Table 3. Cross sections in $\mathrm{pb}$ for $e^{+}-e^{-}$annihilation into hadrons at LEP and deep-inelastic positron-proton scattering at HERA as calculated in the POWHEG framework and in a conventional fixed order NLO calculation [78].

\begin{tabular}{|c|c|c|c|c|c|c|c|}
\hline \multicolumn{2}{|c|}{} & \multicolumn{2}{|c|}{$p \bar{p} \rightarrow W^{+}+X$} & \multicolumn{2}{c|}{$p \bar{p} \rightarrow Z+X$} & \multicolumn{2}{c|}{$p p \rightarrow h+X$} \\
\cline { 2 - 8 } \multicolumn{2}{|c|}{} & \multicolumn{2}{|c|}{$\begin{array}{c}E_{\mathrm{cms}}=1.8 \mathrm{TeV} \\
m_{\ell \nu}>10 \mathrm{GeV}\end{array}$} & \multicolumn{2}{c|}{$\begin{array}{c}E_{\mathrm{cms}}=1.96 \mathrm{TeV} \\
66\end{array} m_{\ell \ell}<116 \mathrm{GeV}$} & \multicolumn{2}{c|}{$\begin{array}{c}E_{\mathrm{cms}}=115 \mathrm{TeV} \\
<m_{\tau \tau}<125 \mathrm{GeV}\end{array}$} \\
\hline$\mu=\mu_{R}=\mu_{F}$ & Factor & POWHEG & NLO & POWHEG & NLO & POWHEG & NLO \\
\hline \multirow{4}{*}{$m_{\ell \nu} / m_{\ell \ell}$} & $1 / 2$ & $1235.4(5)$ & $1235.1(1.0)$ & $243.96(14)$ & $243.84(16)$ & $2.3153(13)$ & $2.3130(13)$ \\
\cline { 2 - 8 } & 1 & $1215.0(5)$ & $1214.9(9)$ & $239.70(13)$ & $239.59(16)$ & $2.4487(12)$ & $2.4474(13)$ \\
\cline { 2 - 8 } & 2 & $1201.4(5)$ & $1202.0(9)$ & $236.72(13)$ & $236.77(15)$ & $2.5811(13)$ & $2.5786(13)$ \\
\hline \multirow{3}{*}{$m_{\perp}$} & $1 / 2$ & $1231.0(5)$ & $1230.3(1.0)$ & $243.00(14)$ & $243.06(16)$ & $2.2873(13)$ & $2.2869(14)$ \\
\cline { 2 - 8 } & 1 & $1211.8(5)$ & $1211.7(9)$ & $239.01(13)$ & $238.96(15)$ & $2.4255(12)$ & $2.4231(19)$ \\
\cline { 2 - 8 } & 2 & $1198.8(5)$ & $1199.3(9)$ & $236.23(13)$ & $236.13(14)$ & $2.5623(13)$ & $2.5620(14)$ \\
\hline
\end{tabular}

Table 4. Cross sections in pb for inclusive $W^{+}\left[\rightarrow e^{+} \nu_{e}\right]$ and $Z\left[\rightarrow e^{+} e^{-}\right]$production at the Tevatron and $h\left[\rightarrow \tau^{+} \tau^{-}\right]$production via a top-quark loop at the LHC as calculated in the PowHEG framework and in a conventional fixed order NLO calculation [78].

parton interactions have been disabled to allow for a study at the parton-shower level. Each $W \rightarrow \ell \nu$ decay is corrected for QED next-to-leading order and soft-resummation effects in the YFS approach [74].

\subsection{Tests of internal consistency}

The aim of this section is to provide consistency checks on the different aspects of the Powheg implementation in Sherpa. At first, total cross sections as obtained from Powheg are compared with the corresponding results from a standard NLO calculation. In this case, the public release SHERPA-1.2.2 $2^{5}$ serves as the reference, which includes an implementation of [78]. Results for $e^{+} e^{-}$annihilation into hadrons and deep-inelastic positron-proton scattering are presented in table 3. Numbers for inclusive Z-boson

\footnotetext{
${ }^{5}$ See http://www.sherpa-mc.de.
} 

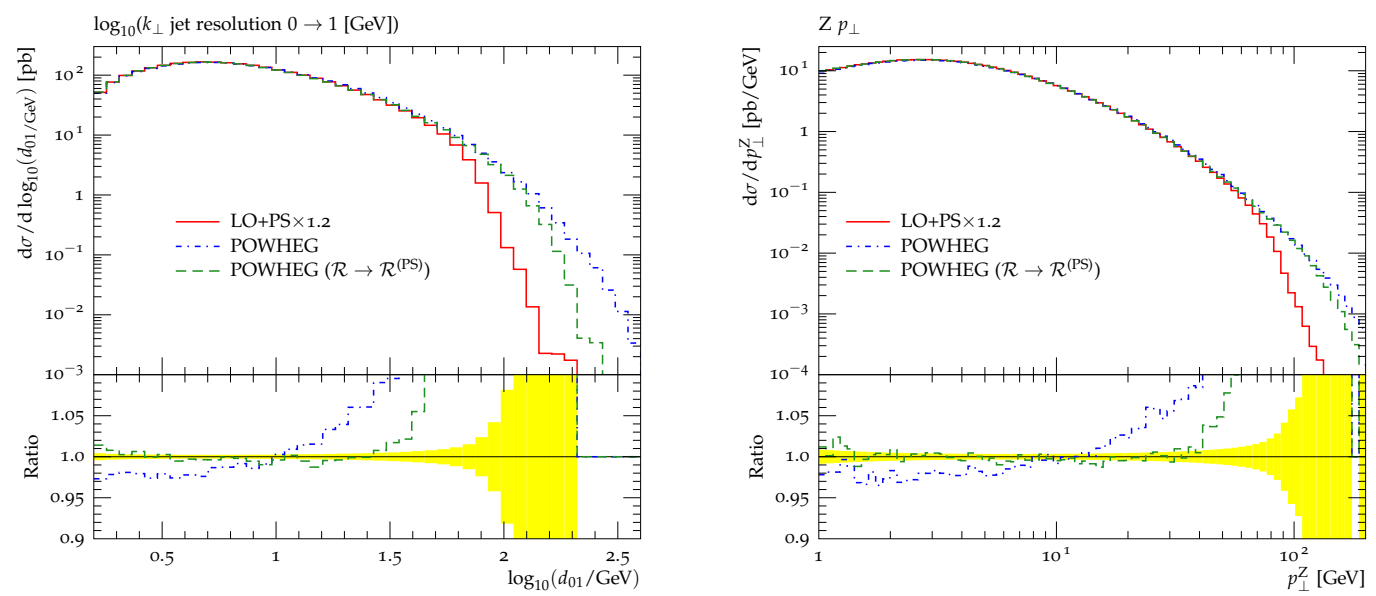

Figure 2. $0 \rightarrow 1$ jet resolution in $k_{T}$ clustered jets and transverse momentum of the reconstructed $Z$-boson in Drell-Yan lepton-pair production at the Tevatron. The standard parton shower effected on the leading order matrix elements (solid, red) is compared to the PowHEG formulation (dotted, blue) and to Powheg with the real emission matrix element $\mathcal{R}$ replaced by its parton-shower approximation $\mathcal{R}^{(\mathrm{PS})}$ (dashed, green).

production with decay to an electron-positron pair, for inclusive $W$-boson production with decay to an electron-neutrino pair, and for Higgs-boson production via a top-quark loop with decay into $\tau$ are listed in table 4 . The agreement between the PowHEg results and those of the standard integration method typically is within a $1 \sigma$ range as given by the respective Monte-Carlo errors.

To examine differences between Powheg and a parton-shower Monte Carlo regarding the exponentiation of the real-emission matrix elements in POWHEG, $\mathcal{R}$ can be approximated by its parton-shower equivalent in eq. (2.21), thus leading to a corrective weight of $w(\{\vec{a}\})=1$. Performing this (seemingly trivial) replacement does not only constitute a mandatory cross-check, whether the parton-shower approximation is retained, but it also estimates the size of corrections that can be expected at all when switching to NLO accuracy in the event simulation. Apart from the overall normalisation, in processes with no additional phase space dependence introduced by the loop matrix element, the emission pattern in Powheg should be identical to the parton-shower result. This is verified in inclusive $Z$ boson production at Tevatron energies as displayed in figure 2. For low transverse momentum (low jet resolution) $p_{\perp} \ll \mu_{F}$ both distributions coincide within statistical errors, which are indicated by the yellow band in figure 2. For large values the emission phase space is severely restricted in the parton-shower approach, as $t<\mu_{F}^{2} \approx m_{Z}^{2}$ and $p_{\perp} \lesssim t$. Any contribution to this phase-space region must therefore originate from configurations where more than one hard parton recoils against the lepton pair. Such configurations are suppressed by higher orders of $\alpha_{s}$, and therefore the emission rate is gravely underestimated by the parton shower. As a direct consequence, all deviations are then manifestations of the exponentiation of non-logarithmic terms, which can be sizeable in the hard wide-angle emission region. 

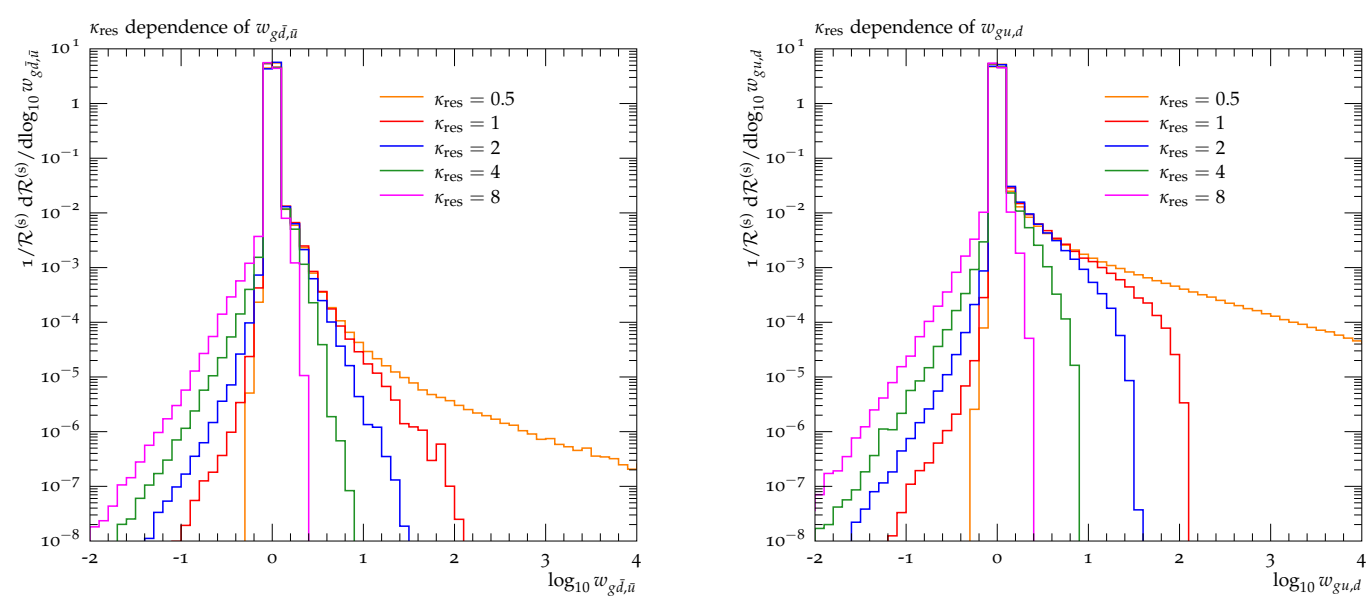

Figure 3. Dependence of the parton shower correction factor $w_{i j, k}$ on the $Z$ - $H$-splitting parameter $\kappa_{\text {res }}$ for $W^{-}$production at the Tevatron.
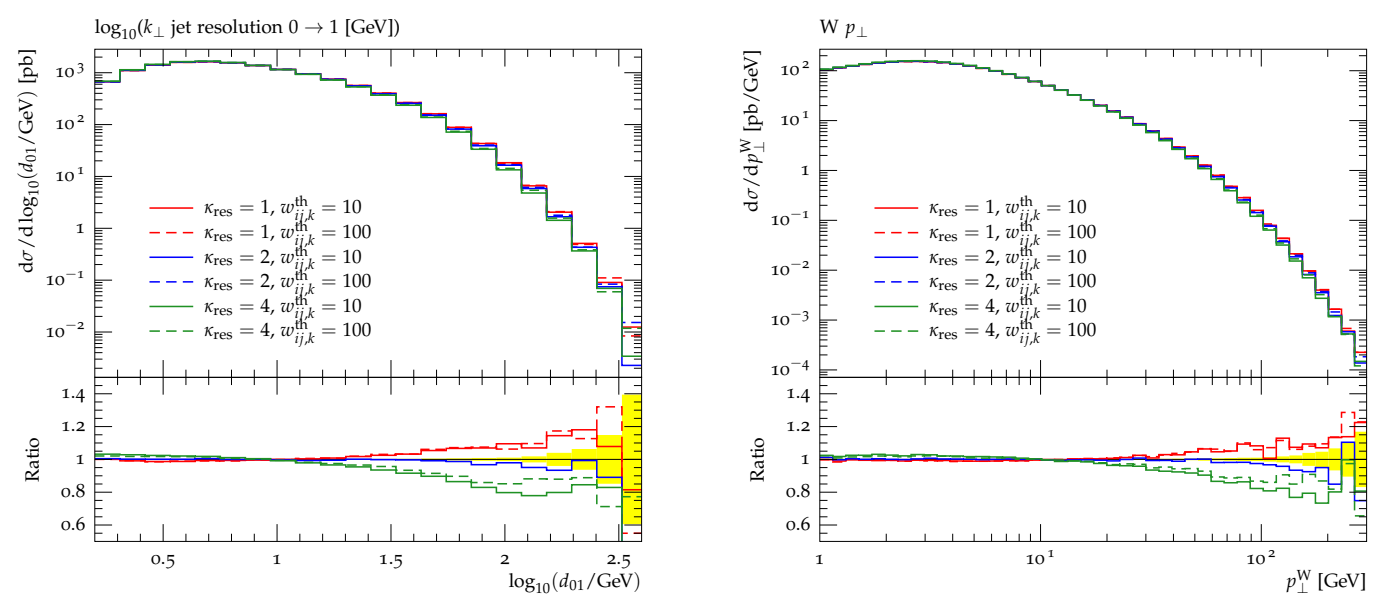

Figure 4. Predictions for $0 \rightarrow 1$ jet resolution in $k_{T}$ clustered jets and transverse momentum of the reconstructed $W$ boson in $W$-boson production at the Tevatron for different settings of the $Z$ - $H$-splitting parameters $\kappa_{\text {res }}$ and $w_{i j, k}^{\text {th }}$.

The automatic splitting of the real-emission matrix element into singular and regular contributions as presented in section 3.3 contains two unphysical parameters: $\kappa_{\text {res }}$, which governs the relative sizes of the exponentiated, singular part $\mathcal{R}^{(\mathrm{s})}$ and the nonexponentiated, regular part $\mathcal{R}^{(\mathrm{r})}$, and $w_{i j, k}^{\mathrm{th}}$, which determines when the above separation is actually employed. The effect of $\kappa_{\text {res }}$ on the central parton shower correction factor $w_{i j, k}$ is detailed in figure 3 . There, it can be seen that for values of $\kappa_{\text {res }}$ chosen neither too low, such that the maximum of the correction factor rises beyond reasonable bounds rendering the reweighting of the parton shower inoperable, nor too high, such that parts of leading logarithmic structure of $\mathcal{R}$ are not exponentiated, event generation with the accuracy aimed at by the Powheg algorithm is feasible. Hence, the results of the Monte-Carlo simulation 

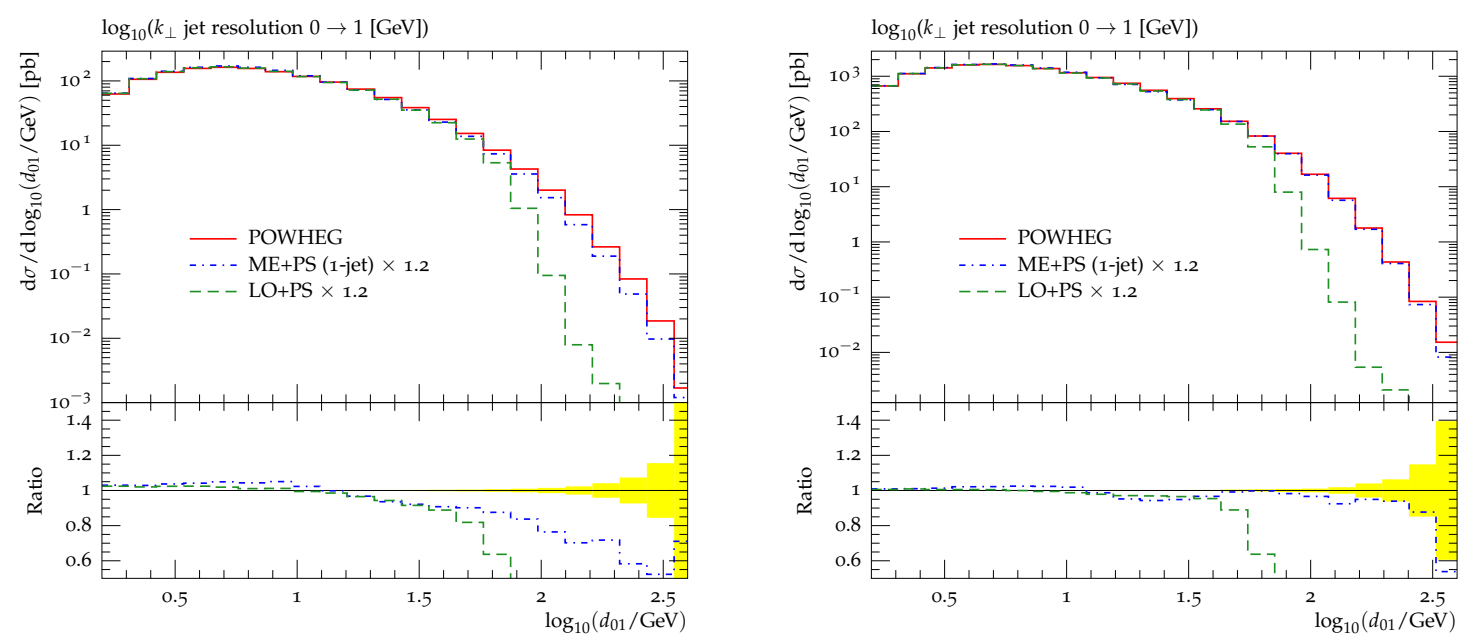

Figure 5. Predictions for the $0 \rightarrow 1$ jet resolution in $k_{T}$ clustered jets in $Z / \gamma^{*}$ (left) and $W$ (right) boson production at the Tevatron.

should be fairly independent of $\kappa_{\text {res }}$ and $w_{i j, k}^{\text {th }}$, if varied within a reasonable range. Figure 4 displays predictions for transverse momentum spectra in $W$-boson production for several values of $\kappa_{\text {res }}$. As expected, no significant variations of the emission pattern can be observed. The small differences that can be seen when changing the resolution scale $\kappa_{\text {res }}$ are entirely within the logarithmic accuracy of the parton-shower approach and therefore also within the logarithmic accuracy of the real-emission contribution in PowHEG. Variations in $w_{i j, k}^{\text {th }}$ only have very little influence on physical distributions.

\subsection{Comparison with tree-level matrix-element parton-shower merging}

By comparing POwHEg results to a standard parton shower combined with LO matrix elements (LO+PS), it can be established whether observables are produced correctly in regions where the soft/collinear approximations in the parton shower are equivalent to the $R / B$ ratios in Powheg. An example is the distribution of the jet resolution scale $d_{01}$, using the longitudinally invariant $k_{T}$-algorithm in $W / Z+$ jets production. This observable amounts to the $k_{T}$-scale where a 1 -jet event is clustered into a 0 -jet event. Figure 5 shows that there is good agreement between the LO+PS and PowHEg results for $d_{01}<50 \mathrm{GeV}{ }^{6}$ For harder emissions the LO+PS approach fails due to the restricted phase space, as explained in the previous section.

In this publication, we regard the PowHEG method as an advanced matrix-element correction technique for the parton-shower algorithm, in which the correction is supplemented with local $K$-factors to implement full NLO corrections. It is therefore useful to compare the respective results with matrix-element parton-shower merged samples (ME+PS), which are rescaled by a suitably chosen global $K$-factor. Such samples are known to yield approximate NLO radiation patterns by effectively implementing higher-order matrix-element cor-

\footnotetext{
${ }^{6}$ Here and in the following, the yellow band indicates the size of statistical errors on the POWHEG event sample.
} 
rections into the parton shower. An implementation of one of the most advanced ME+PS algorithms to date is available within the SHERPA framework [17] such that a direct comparison with PowHEg is a straightforward exercise. However, because of the lack of virtual contributions in the LO+PS and ME+PS samples, an agreement of the total rate cannot be expected. Thus, in the comparisons below the following global $K$-factors were employed:

- $K=1.038$ for $e^{+} e^{-} \rightarrow$ hadrons at LEP energies,

- $K=1.2$ for $Z / \gamma^{*}$ and $W$ production at Tevatron energies,

- $K=1.2$ for $Z Z$ production at the $\mathrm{LHC}(14 \mathrm{TeV})$,

- $K=1.34$ for $W^{+} W^{-}$production at the LHC $(14 \mathrm{TeV})$, and

- $K=2.1$ for Higgs production through gluon fusion at the same LHC energies.

When comparing PowHEg results to ME+PS results including matrix elements up to the 1-jet final state one should obtain a very similar radiation pattern. The observed agreement indeed is very good, as expected. Figure 5 shows that, for example, the differential one-jet rates in $W / Z$-boson production agrees on the $20 \%$ level, even for relatively large scales $\left(d_{01}>50 \mathrm{GeV}\right)$. The remaining differences can be attributed to the differences in the Sudakov form factors: While PowHEg exponentiates $R / B$, the ME+PS method uses standard Sudakov form factors at the logarithmic accuracy of the parton shower.

Such differences become visible also in the multiplicity distribution of $k_{T}$ jets with $p_{\perp}>20 \mathrm{GeV}$ in Drell-Yan and $W$ production, cf. figure 6 . The 0 -jet and 1 -jet rates agree within 10\% between POWHEG and ME+PS, but for higher multiplicity final states the Powheg method predicts significantly more jets. Here a ME + PS simulation with more jets in the matrix element would lead to better agreement.

Now focussing on the properties of the leading jet produced in association with a $W$ or $Z$ boson, the transverse momentum of the leading jet is shown in figure 7 . Here the LO+PS approach fails to describe the hard tail of the distribution, again due to lacking phase space, while the PowHEG and ME+PS approaches agree within $20 \%$. The separation in $\eta-\phi$ space between this jet and the $W / Z$ boson is displayed in figure 8. Clear differences are observed in the shape of the distribution when comparing the LO+PS approach with both PowHEG and ME + PS. This is expected, since parton showers cover only a restricted area of the phase space, and, in addition, they do not encode the full final-state correlations described by the matrix elements. On the other hand, results from the PowHEG and ME+PS methods agree very well, with differences below $10 \%$ only.

The transverse momentum of the Higgs boson and the transverse momentum of the leading jet displayed in figure 9 give a similar picture as in vector boson production: All three methods agree very well for low transverse momenta. In the high $p_{\perp}$ region the Powheg and ME+PS approaches show differences up to $40 \%$.

Figure 10 shows that minor differences arise between the LO+PS and the POwHEG and ME+PS approaches in the pseudorapidity spectrum of the leading jet. This can be understood as a direct consequence of the different transverse momentum distributions 
in the $\mathrm{LO}+\mathrm{PS}$ method, as harder jets tend to be more central than softer ones. The Powheg and ME+PS approaches agree well in the central rapidity region and show up to $10 \%$ difference only in the forward region. The distribution of $\eta$ - $\phi$ separation between the two leading jets proves again that the PowHEG and ME+PS predictions are very similar, with deviations below the $5 \%$ level. Again, the LO+PS prediction shows a slightly different behaviour, because of the reasons stated above.

Now we turn to look at diboson production at nominal LHC energies of $14 \mathrm{TeV}$. Figure 11 (left) shows a comparison of the scalar sum $H_{T}$ of the transverse momenta of jets and leptons in $Z$-pair production. Deviations of up to $50 \%$ become visible between the three compared approaches. This is especially true in the high- $H_{T}$ region. It is well understood that the predictions of the LO+PS approach are softer than either of the two other approaches, due to the restricted emission phase space. The relatively large differences between the ME+PS approach and POWHEG are naively not expected, but might stem from using consistent but somewhat oversimplified scale schemes. This surely should be analysed in more detail, in a forthcoming publication, where pair production processes, including $W H$ and $Z H$ would be studied. The transverse momentum distributions of the individual $Z$ bosons (figure 11 right) on the other hand agree very well in both approaches, while it is again obvious that the $\mathrm{LO}+\mathrm{PS}$ sample cannot describe the hard region of this spectrum.

In the azimuthal separation of the two $Z$ bosons, see figure 12, a similar feature as in $H_{T}$ can be found: The events are harder in ME+PS than in PowHEG, leading to increased decorrelation of the boson pair. In figure 12 (right) it can be seen that the angle between the boson decay planes is predicted very consistently in all three approaches.

Properties of the leading jet in $W^{+} W^{-}$pair production at LHC energies are displayed in figure 13. On the left hand side one can see the transverse momentum of the leading jet and on the right hand side the separation between lepton and leading jet. For both the ME+PS and POWHEG approaches agree well within 20\% and the LO+PS sample severely underestimates the hardness of the first jet due to its phase-space restrictions.

Figure 14 displays observables related to the two oppositely charged leptons from the two decays. The pseudorapidity difference (left) agrees within $20 \%$ for all three approaches, while their azimuthal decorrelation is significantly lower in the LO+PS sample than in the ME + PS and POwHEG approaches, which agree very well.

\subsection{Comparison with experimental data}

The remainder of this section is dedicated to a comparison of results from the PowheG approach with experimental data to assert the improved description of data, provided by this method.

For the reaction $e^{+} e^{-} \rightarrow$ hadrons at LEP1 energies the LO+PS and ME+PS predictions do not show significant differences except in extreme regions of phase space. The Powheg prediction confirms that picture. This is largely due to the fact that the partonshower algorithm, which is employed in SHERPA is based on Catani-Seymour subtraction terms and those terms constitute a rather good approximation to the real-emission matrix element in the process $e^{+} e^{-} \rightarrow q \bar{q} g$. 

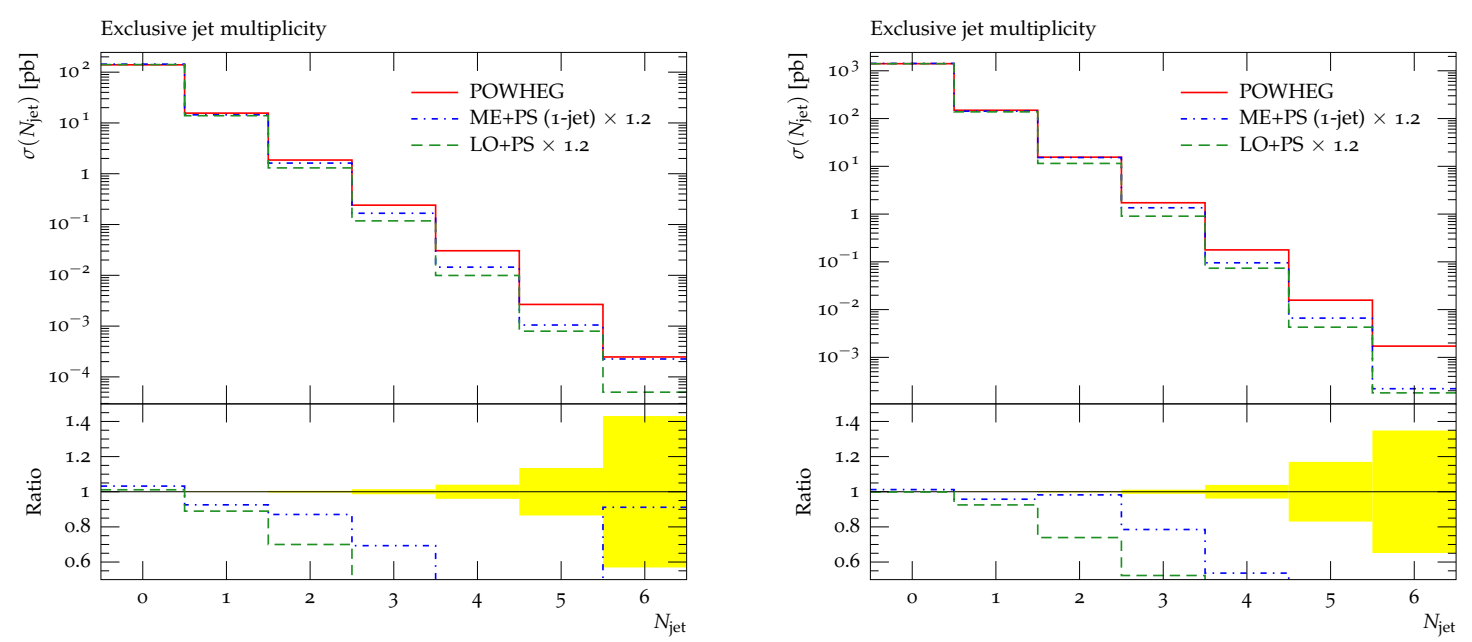

Figure 6. Exclusive jet multiplicity for jets with $p_{\perp}>20 \mathrm{GeV}$ in the $k_{T}$ algorithm in $Z / \gamma^{*}$ (left) and $W$ (right) boson production at the Tevatron.
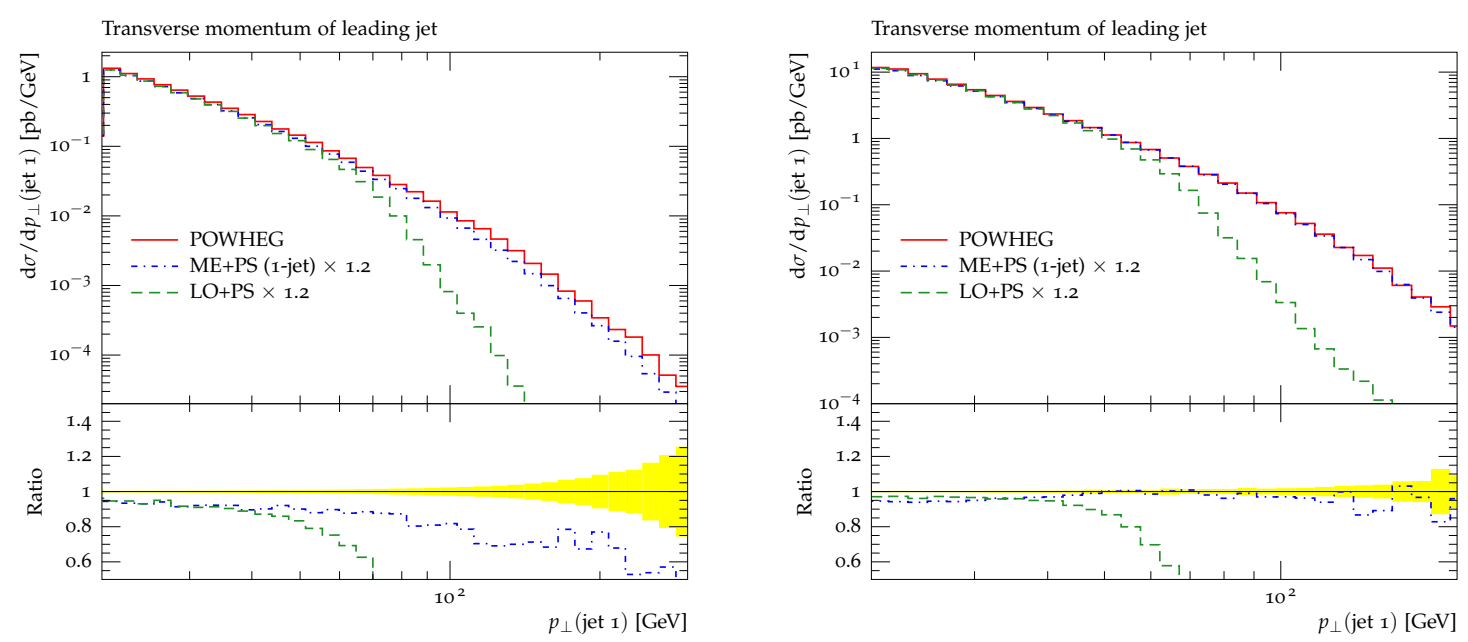

Figure 7. Transverse momentum of the leading jet in $Z / \gamma^{*}$ (left) and $W$ (right) boson production at the Tevatron.

In the distribution of the Durham jet resolution at which 3-jet events are clustered into 2-jet events (figure 15 left) all three approaches agree very well with the measurement over large parts of the phase space. Only in the hard emission region $y_{23}>0.05$ deviations from the LO+PS result can be seen. It is encouraging, although not surprising to note that both Powheg and ME+PS describe the data better. Good agreement of all three approaches with each other and with the measurement is also observed e.g. for the thrust distribution (figure 15 right), the total jet broadening (figure 16 left) and the C-Parameter (figure 16 right).

As was discussed in [86], deep-inelastic scattering processes offer the opportunity to test perturbative QCD in a region where the factorisation scale of the inclusive process, 

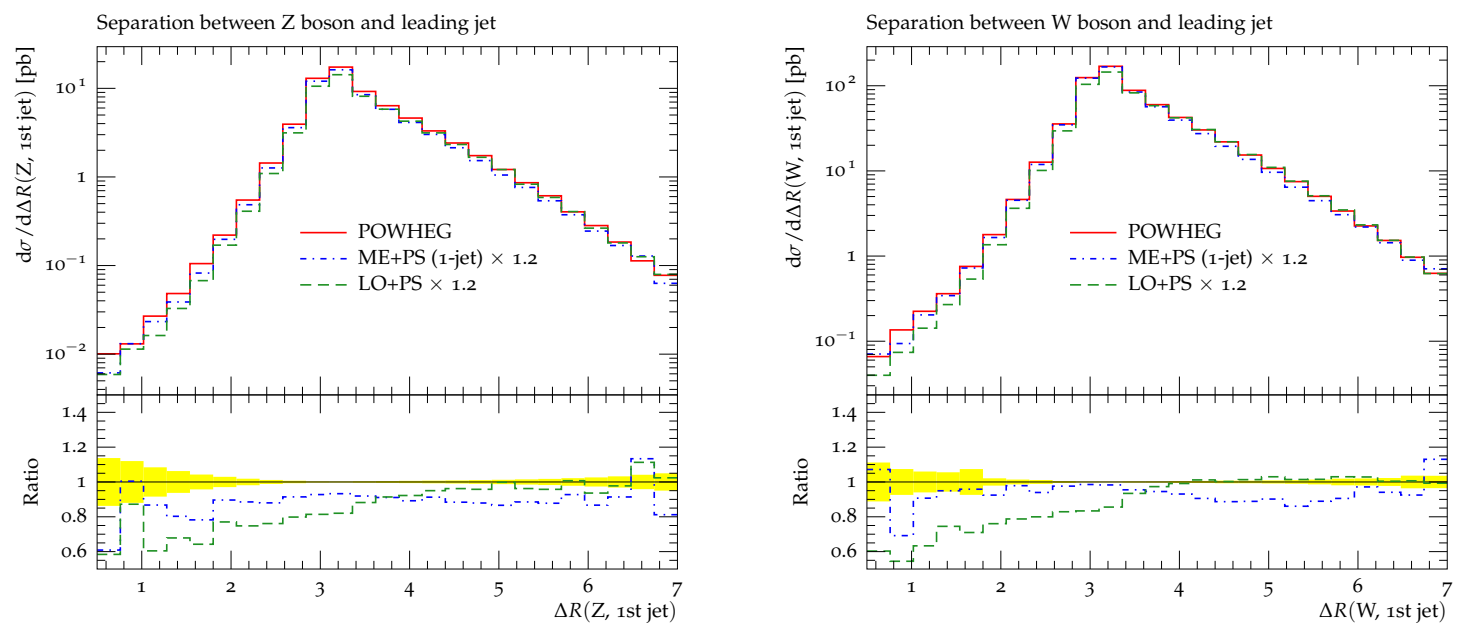

Figure 8. Separation between vector boson and leading jet in $Z / \gamma^{*}$ (left) and $W$ (right) boson production at the Tevatron.
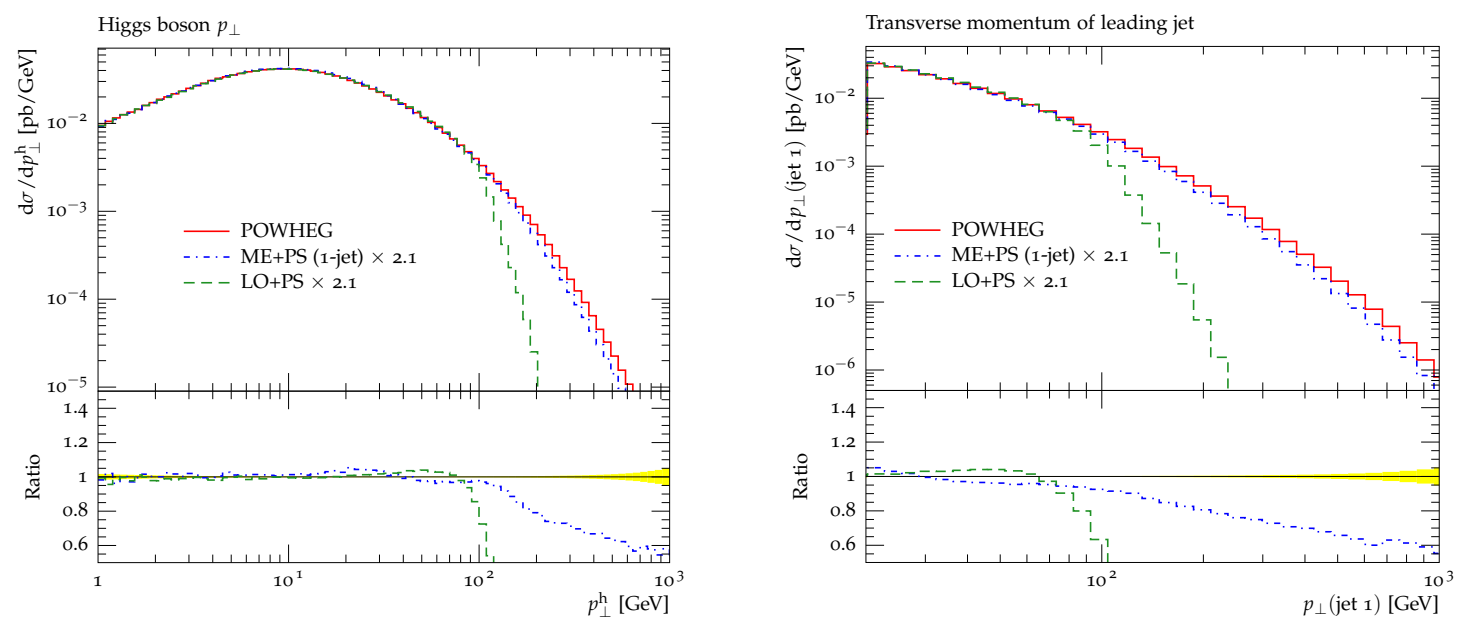

Figure 9. Transverse momentum of the Higgs boson (left) and leading jet (right) in $g g \rightarrow h$ fusion at nominal LHC energies.

$Q^{2}$, is smaller than the scale of the actual event, which might be set e.g. by the transverse momentum of a hard jet. As measurements can be performed down to very low values of $Q^{2}$, many hard jets must usually be included in the simulation to achieve a good description of data throughout the phase space. This method cannot be used in the context of this work, as the POWHeg implementation in SHERPA can so far only be employed for the core process $e^{ \pm} q \rightarrow e^{ \pm} q$. Therefore, results are presented for the high- $Q^{2}$ region only and the discussion of the low- $Q^{2}$ domain is postponed to a forthcoming publication [47], where the merging of PowHeg samples with higher-multiplicity matrix elements will be discussed. Figure 17 shows reasonable agreement between the PowHEG results and experimental data in a measurement of the di-jet cross section performed at the H1 experiment $[101,102]$. 

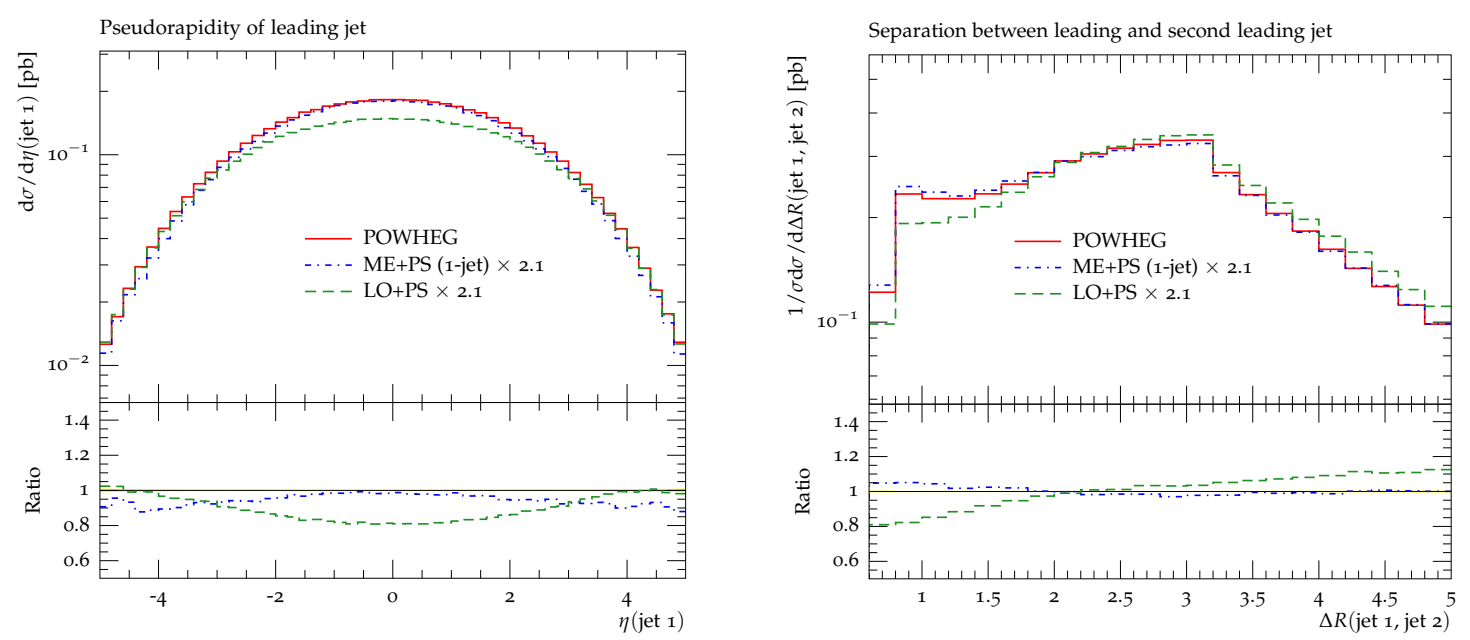

Figure 10. Rapidity of the leading jet (left) and separation of the leading and second-leading jet (right) in $g g \rightarrow h$ fusion at nominal LHC energies.
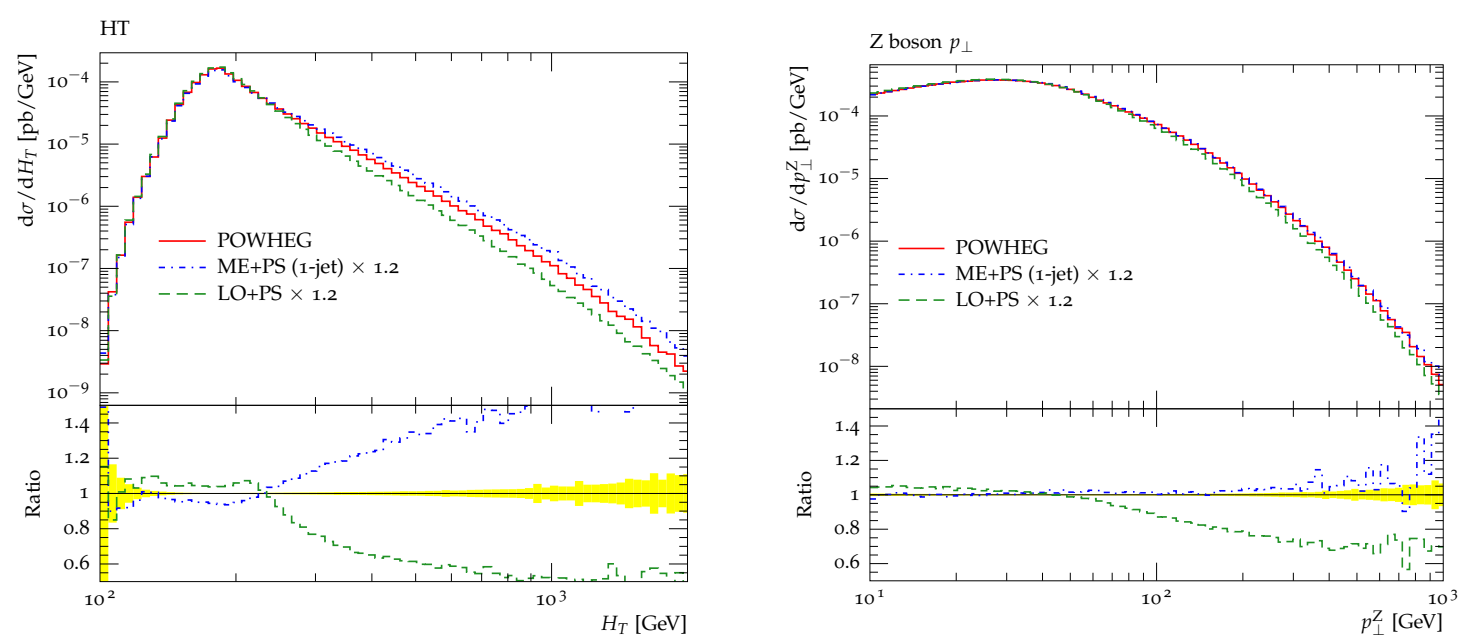

Figure 11. $H_{T}$ (left) and transverse momentum of the individual $Z$ bosons (right) in $Z Z$ production at nominal LHC energies.

Deviations from the LO+PS result are apparent, especially at lower values of $Q^{2}$, as the phase space of the parton shower is severely restricted by the low factorisation scale. Similar findings apply to the rapidity spectra shown in figure 18 .

The probably most discussed observable probing the influence of QCD radiation in hadron-hadron collisions is the transverse momentum of the lepton pair in Drell-Yan production, which is displayed in figure 19. Very good agreement, compared to a recent measurement, is found for both the PowHEG and ME+PS approaches, while the LO+PS method is not able to describe large parts of the spectrum because of the restricted real-emission phase space. The rapidity of the $\mathrm{Z}$ boson in figure 19 is described very well by all three approaches. 

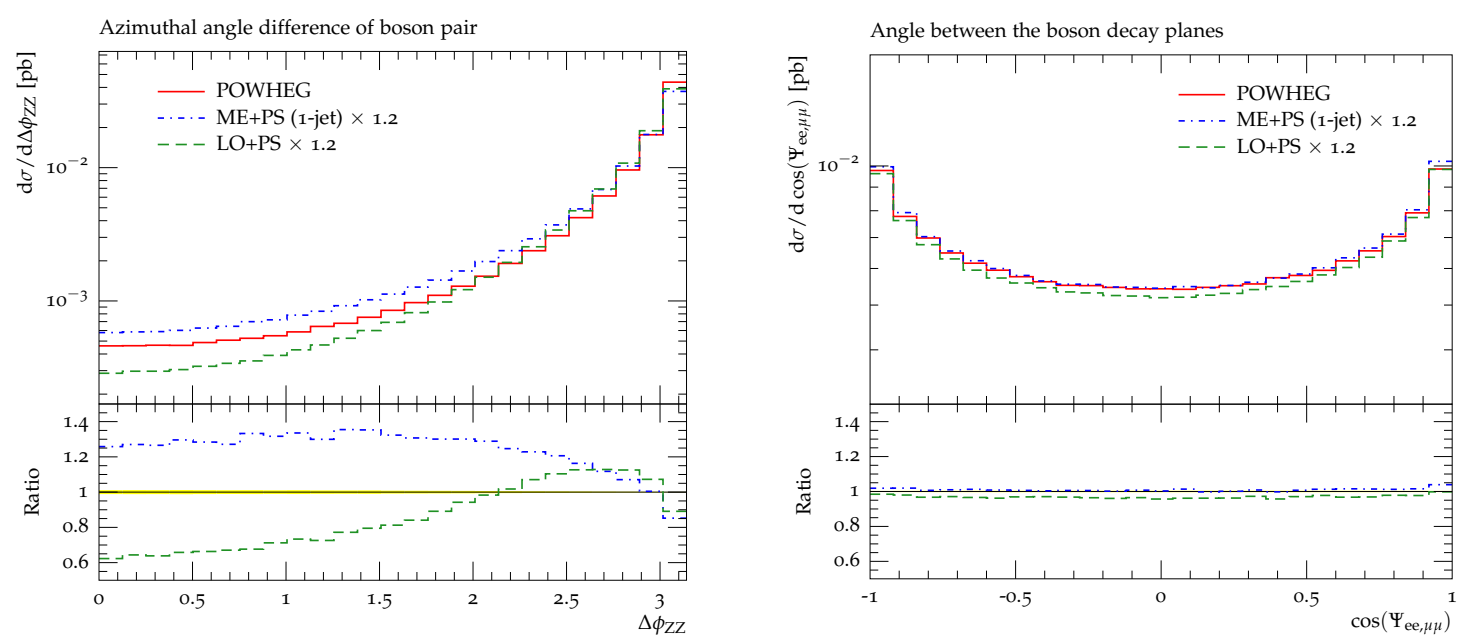

Figure 12. Azimuthal angle between the two $Z$ bosons (left) and angle between the two $Z$ decay planes (right) in $Z Z$ production at nominal LHC energies.
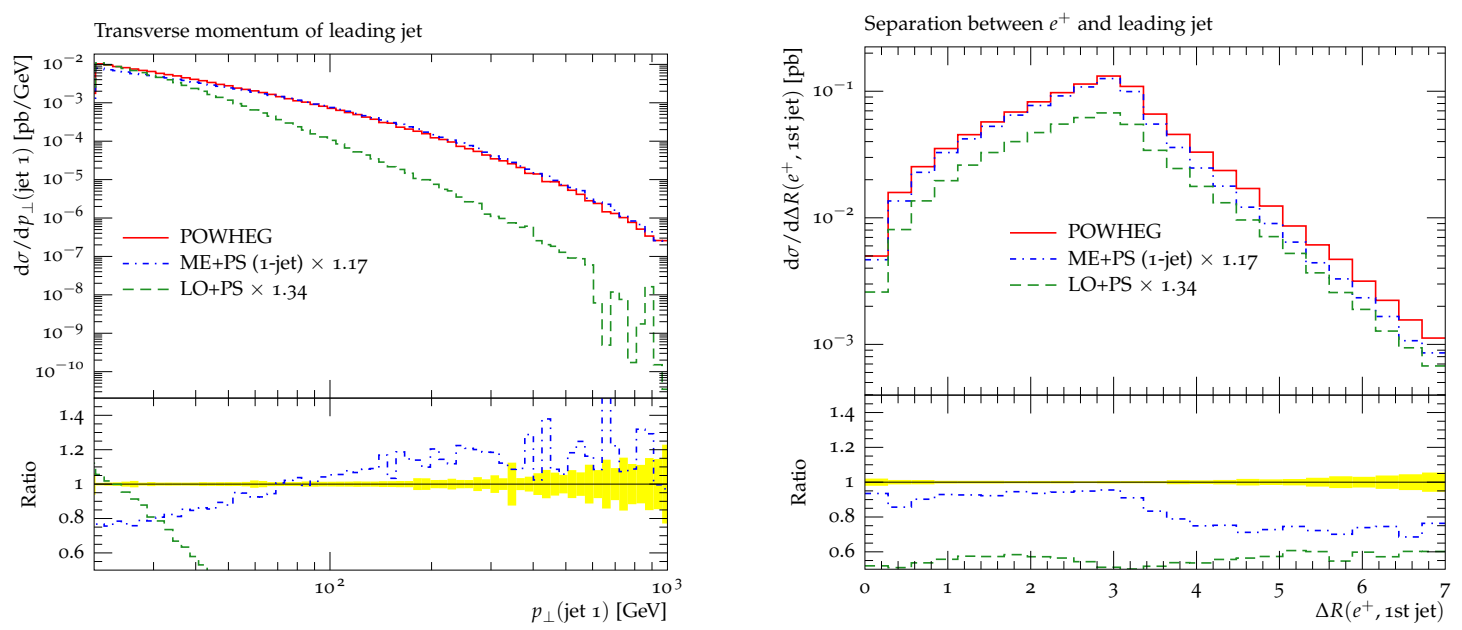

Figure 13. Transverse momentum of the leading jet (left) and separation of the lepton and leading jet (right) in $W^{+} W^{-}$production at nominal LHC energies.

The situation is very similar in $W$-boson production. A comparison of PowHEG predictions with Tevatron data from the $\mathrm{D} \varnothing$ experiment [103] is shown in figure 20, where very good agreement between the Monte-Carlo result and the data can be observed. In addition to the direct comparison the uncertainties related to a variation of the renormalisation and factorisation scales are also shown. Thereby, two different strategies are pursued: While the inner (dark) band shows the uncertainty related to a variation of the scale in the hard matrix elements only, the outer (light) band shows the influence of varying the scales also in the parton-shower evolution. It is rather obvious that the latter approach yields the larger variations, as it is associated with an uncertainty in the choice of the strong coupling for the real-emission subprocess. While this process essentially determines the shape of the 

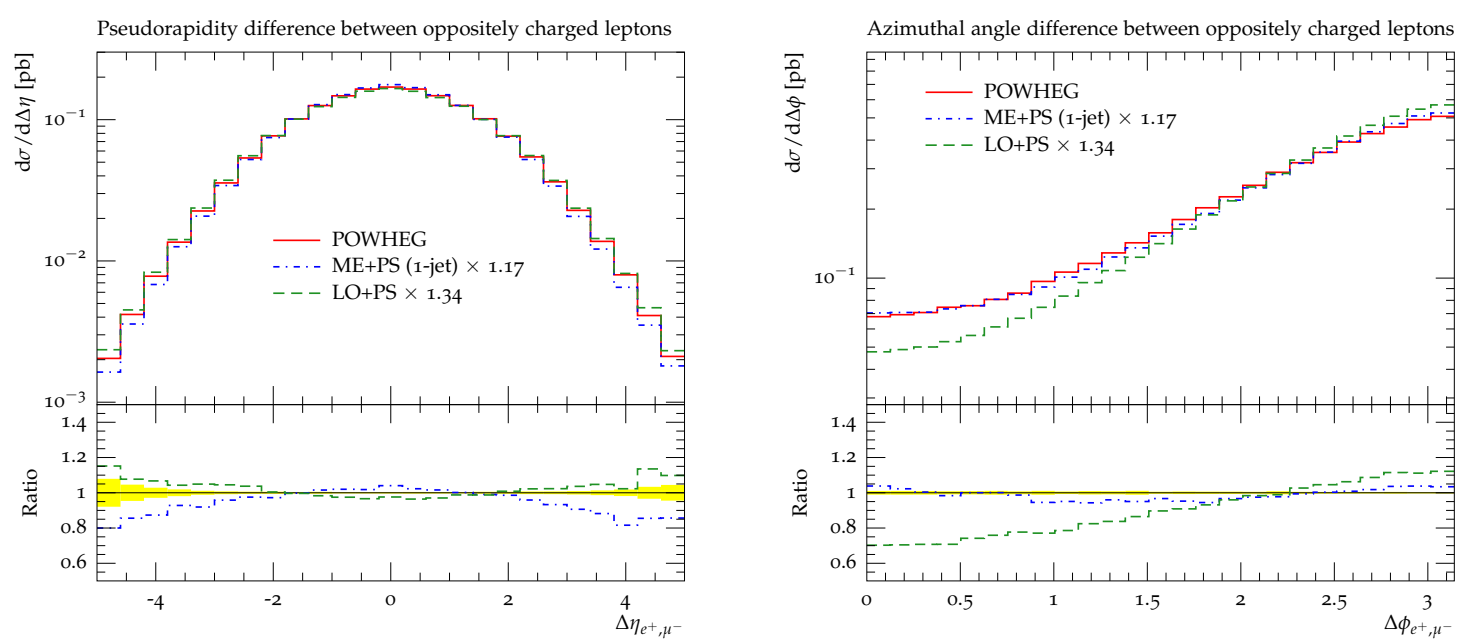

Figure 14. Pseudorapidity difference (left) and azimuthal angle (right) between the two oppositely charged leptons in $W^{+} W^{-}$production at nominal LHC energies.
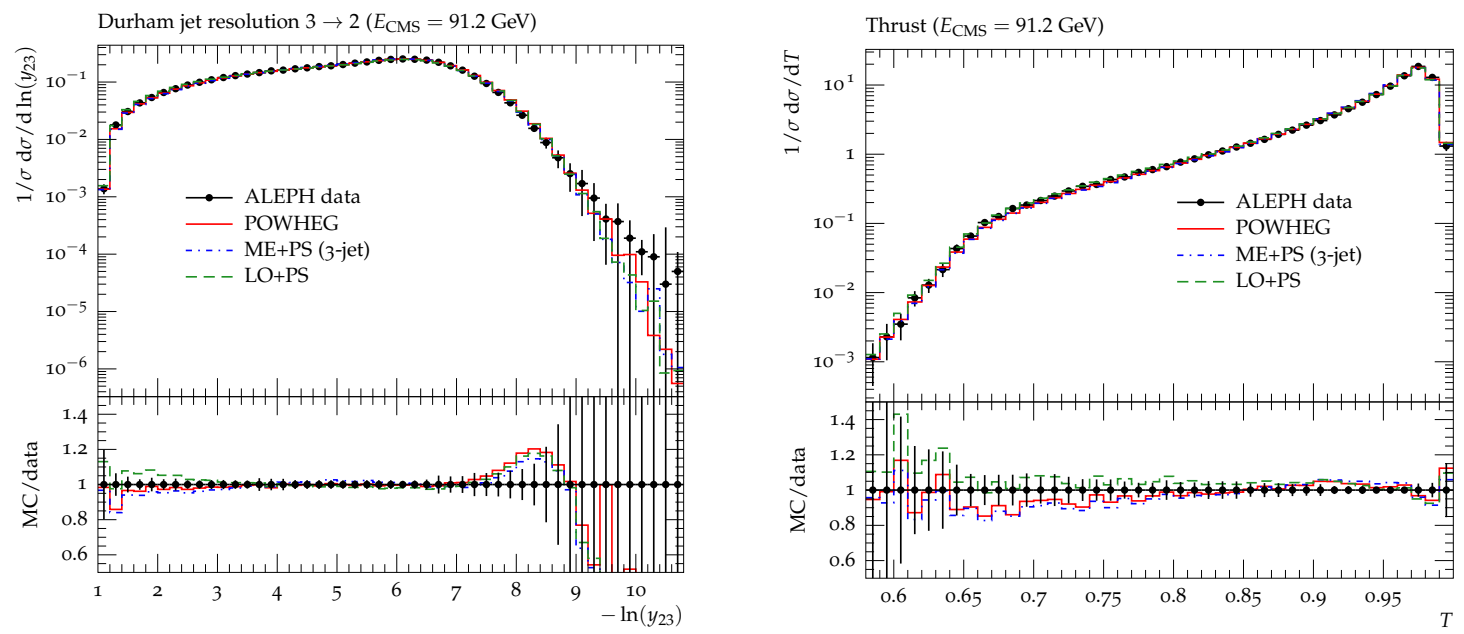

Figure 15. Durham $2 \rightarrow 3$ jet resolution (left) and thrust distribution (right) compared to data from the ALEPH experiment [104].

transverse momentum distribution in figure 20, it enters at tree-level accuracy only, thus leading to a rather large scale dependence.

\subsection{Comparison with existing POWHEG results}

In this section the predictions of our automated implementation are compared to results from the dedicated codes in the HERWIG ++2.4 .2 event generator $[7,8] .{ }^{7}$ We analyse Drell-Yan lepton pair production and Higgs boson production through gluon-gluon fusion. HERWIG + + was set up to use the CTEQ6.6 [87] PDF and matrix element cuts have been

\footnotetext{
${ }^{7}$ We would like to thank David Grellscheid, Keith Hamilton and Peter Richardson for their help with settings in the HeRwiG++ generator run card.
} 

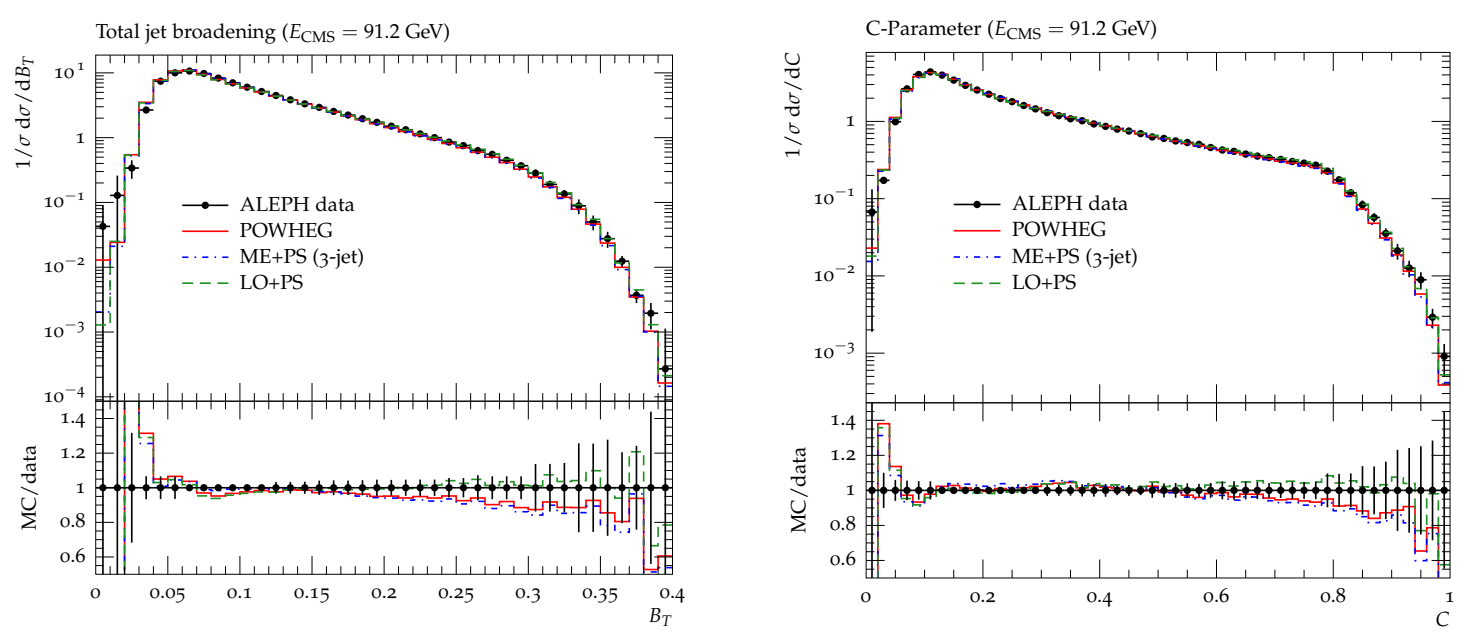

Figure 16. Total jet broadening (left) and C-Parameter (right) compared to data from the ALEPH experiment [104].

chosen identical to the ones employed in SHerpa. The same analyses as for Sherpa have been performed on the HeRwIG++ events and serve as a basis for the following discussion. Corresponding details can be found in section 4.3 and 4.4 .

For Drell-Yan lepton pair production we first compare predictions of inclusive observables related to the lepton pair in figures 21-23. Excellent agreement is found for the mass and rapidity of the lepton pair as well as the pseudorapidity of the individual leptons. Looking at the transverse momentum of both, individual leptons and the lepton pair, one finds the SHERPA predictions to be slightly harder, which also seems to be favoured by the $\mathrm{D} \varnothing$ data shown in figure 23 .

Further distributions sensitive to the hard radiation pattern are displayed in figures 2426. The transverse momentum spectrum for the leading jet is consistent for both implementations over large regions of phase space. The pseudorapidity of the leading jet shows good agreement in the central region, while SHERPA produces more jets in the forward direction, $|\eta|>3$. Regarding the leading jet, we find excellent agreement for both the inclusive jet multiplicity and the jet resolution scale. For higher jet multiplicities one can see differences, with SHERPA producing more jets. At this point, it is important to stress that the production of multiple hard jets is described by the parton shower only, and the respective prediction is not systematically improved by the PowHEG method. A perfect agreement of the two results would therefore be rather unexpected, given the different assumptions underlying the parton shower algorithms in SHERPA and Herwig++. Angular correlations between the lepton pair and the leading jet show some deviations especially in the region of low separation in $\Delta R$ and $\Delta \phi$ where multiple hard emissions become relevant. Here the $\mathrm{D} \varnothing[107]$ data also favour the predictions from SHERPA.

For $g g \rightarrow h$ fusion at nominal LHC energies we are comparing HeRwIG ++ to two different settings of SHERPA: One with the correct dependence on the mass of the top quark 

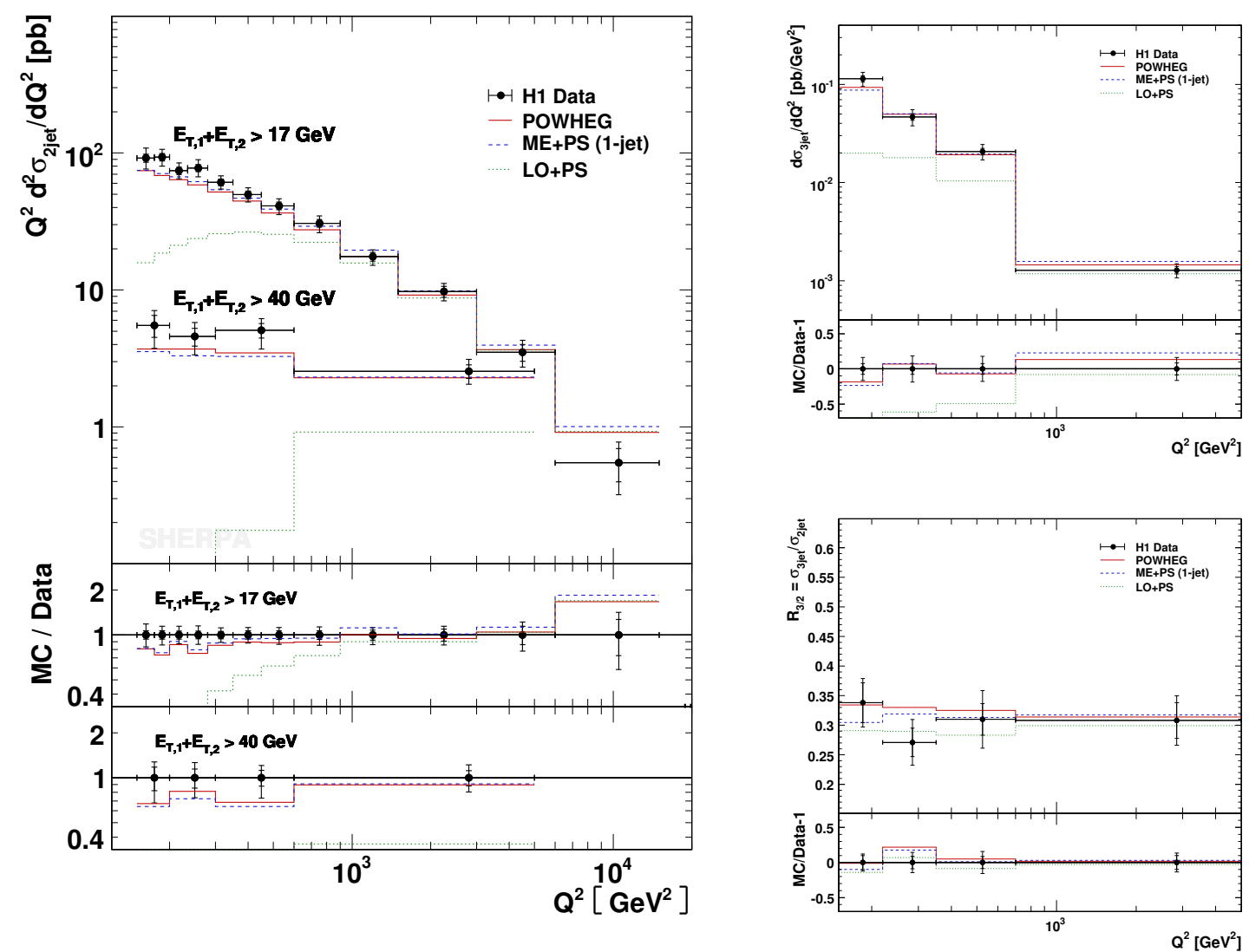

Figure 17. The di-jet cross section as a function of $Q^{2}$ in bins of $E_{T, 1}+E_{T, 2}$ (left), the three-jet cross section as a function of $Q^{2}$ (right top) and the ratio of the three- over the two-jet rate as a function of $Q^{2}$ (right bottom) compared to data from the H1 experiment [101, 102].

in the effective $g g \rightarrow h$ vertex and one with the approximation $m_{t} \rightarrow \infty$ which has also been used for the plots in section 4.3. In figures 27-29 one finds agreement of the inclusive cross section on the level of a few percent between the generators using a finite top quark mass, while the $m_{t} \rightarrow \infty$ approximation leads to a change of $\approx 5 \%$ in the total rates. The shape of the rapidity distribution of the reconstructed Higgs boson shows good agreement while its transverse momentum is considerably harder in the SHERPA predictions. A very similar feature can be found for the transverse momentum of the leading jet. The results for Drell-Yan lepton pair production at the Tevatron showed the same behaviour, albeit not as drastic as observed here. Good agreement is found for the shape of the pseudorapidity of the first jet. The jet multiplicity distribution shows a significant difference for the production of multiple hard jets where the difference between the parton showers in both generators becomes important. For the distribution of the rapidity difference between the reconstructed boson and the leading jet we find good agreement in shape. 

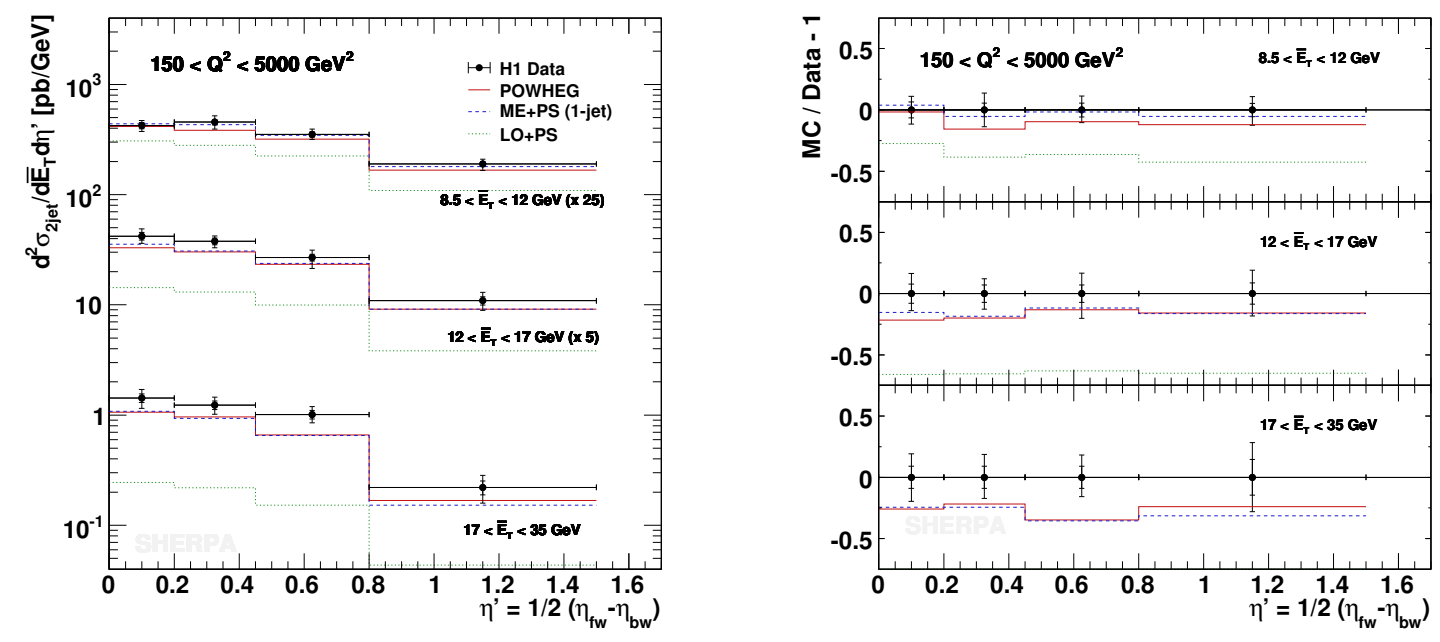

Figure 18. The di-jet cross section as a function of $\eta^{\prime}$, compared to data from the H1 experiment [101]. $\eta^{\prime}$ denotes half the rapidity difference of the two leading jets in the Breit frame.
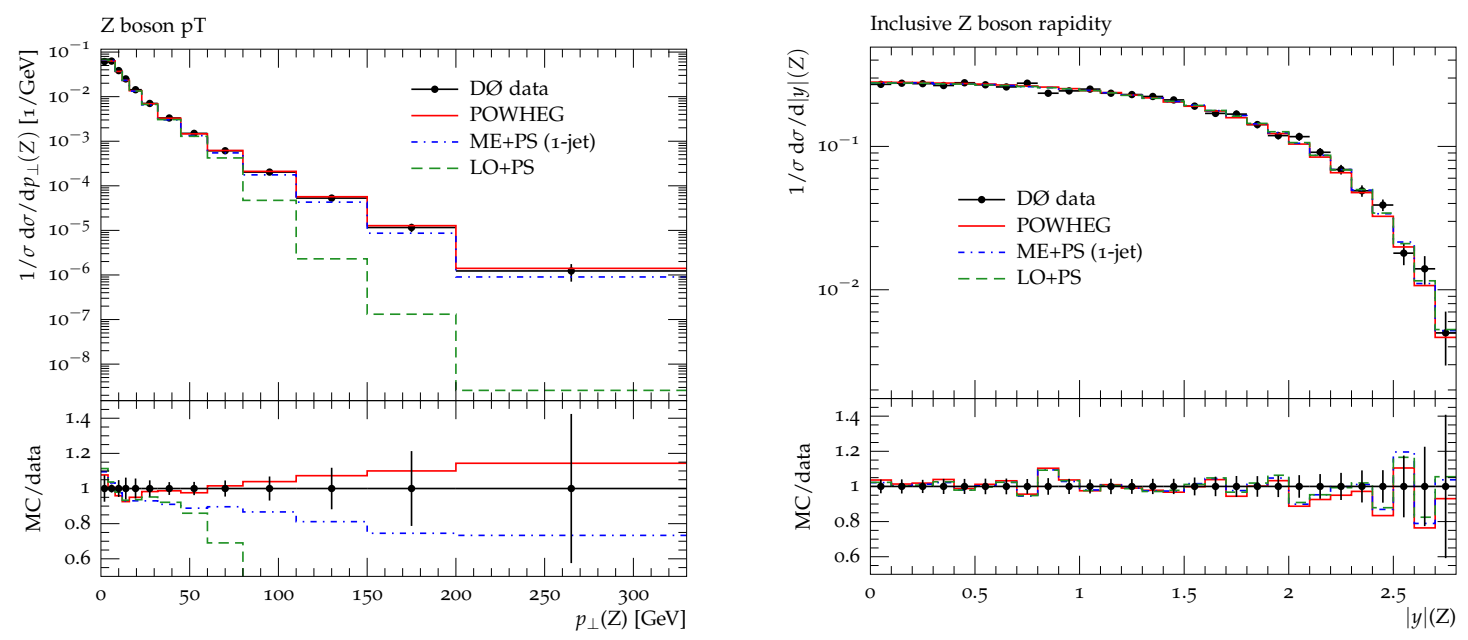

Figure 19. Transverse momentum and rapidity of the $Z$ boson in Drell-Yan lepton-pair production at the Tevatron compared to data from the DØ experiment [105, 106].

\section{Conclusions and outlook}

In this publication the successful implementation of the POWHEG algorithm into the SHERPA framework was reported. The program is fully automated, relying on SHERPA's efficient matrix-element generation modules, which allow to construct real-correction terms for given processes and their respective Catani-Seymour dipole terms in both differential and integrated form. It is worth stressing that this is the first time that the PowheG method has been applied simultaneously to various higher-order calculations using CataniSeymour dipole terms for partitioning the real-emission phase space. This implementation 

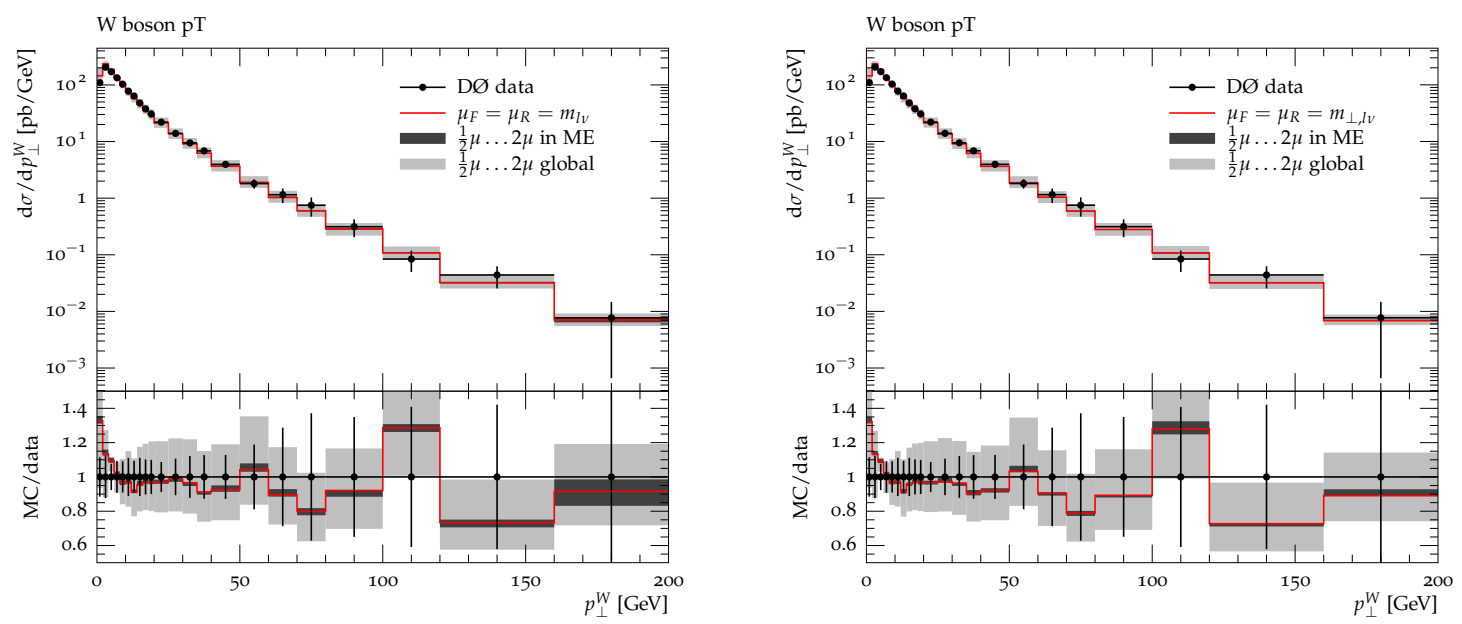

Figure 20. Transverse momentum of the $W$ boson in $W+$ jets production at the Tevatron compared to data from the D $\varnothing$ experiment [103]. Scale variations of the PowHEG prediction by factors of $1 / 2$ and 2 are displayed for two different scale schemes, $\mu_{F}=\mu_{R}=m_{l \nu}$ (left) and $\mu_{F}=\mu_{R}=m_{\perp, l \nu}$ (right). The inner (dark) band displays the variations associated with redefining the scales for matrix elements alone, while the outer (light) band also takes variations in the running coupling of the parton shower evolution into account.
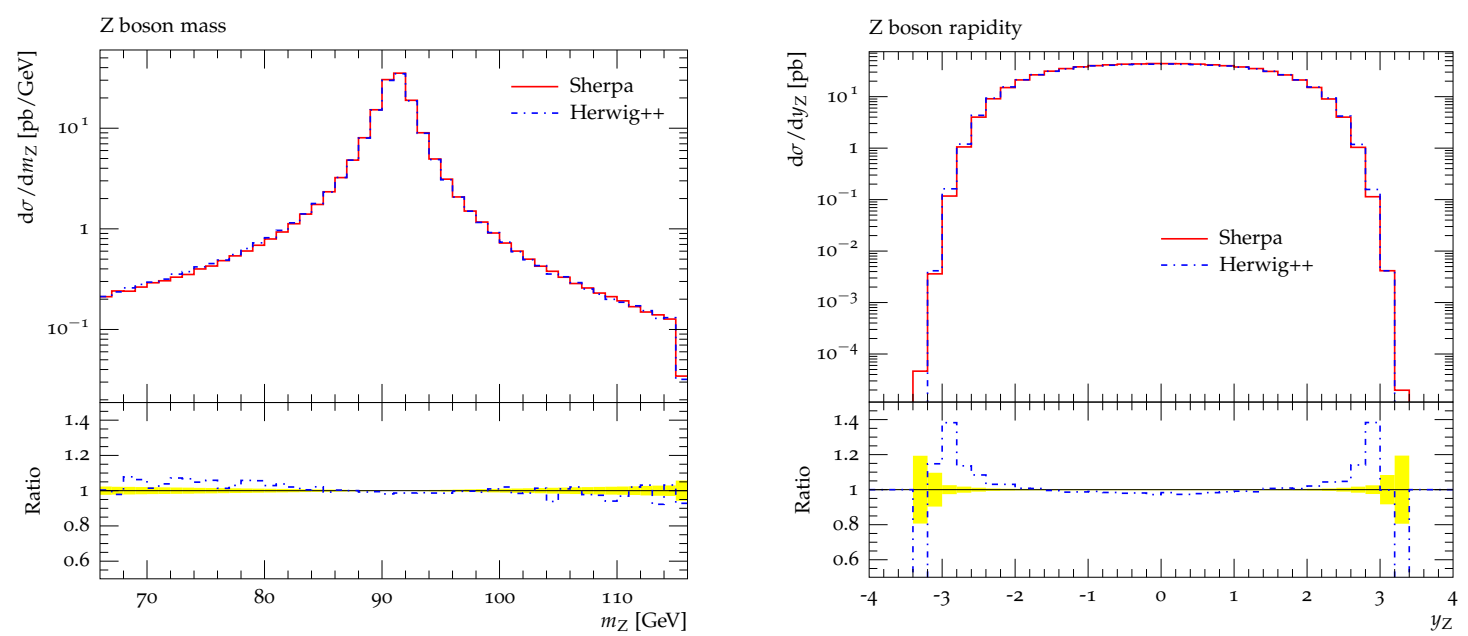

Figure 21. Invariant mass (left) and rapidity (right) of the lepton pair in $Z / \gamma^{*}$ production at the Tevatron.

makes a number of processes, computed at NLO and available in public program libraries accessible for matching with a parton shower. Additional processes are easily added in SHERPA, by merely linking the corresponding code for the virtual correction terms. ${ }^{8}$

The implementation was validated by a number of systematic checks, including

\footnotetext{
${ }^{8} \mathrm{~A}$ wealth of processes not discussed yet is, for instance, available in the extremely well-developed MCFM library.
} 

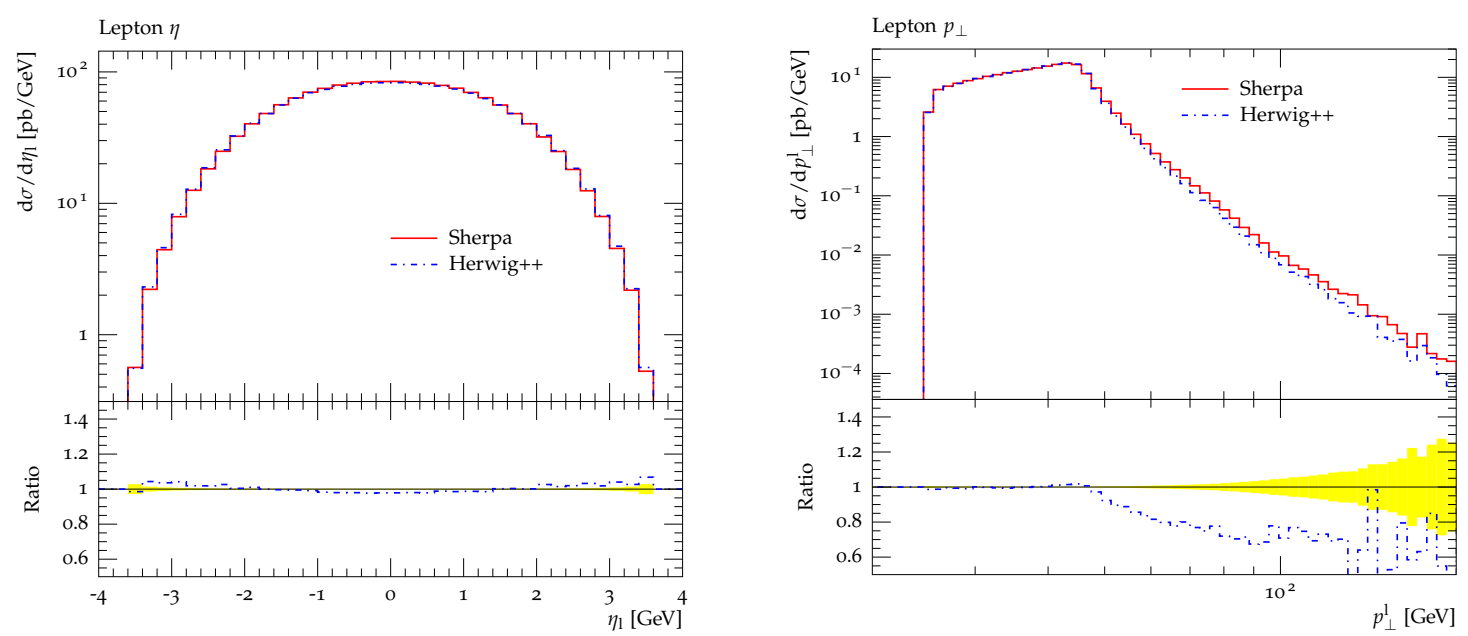

Figure 22. Pseudorapidity (left) and transverse momentum (right) of the lepton in $Z / \gamma^{*}$ production at the Tevatron.
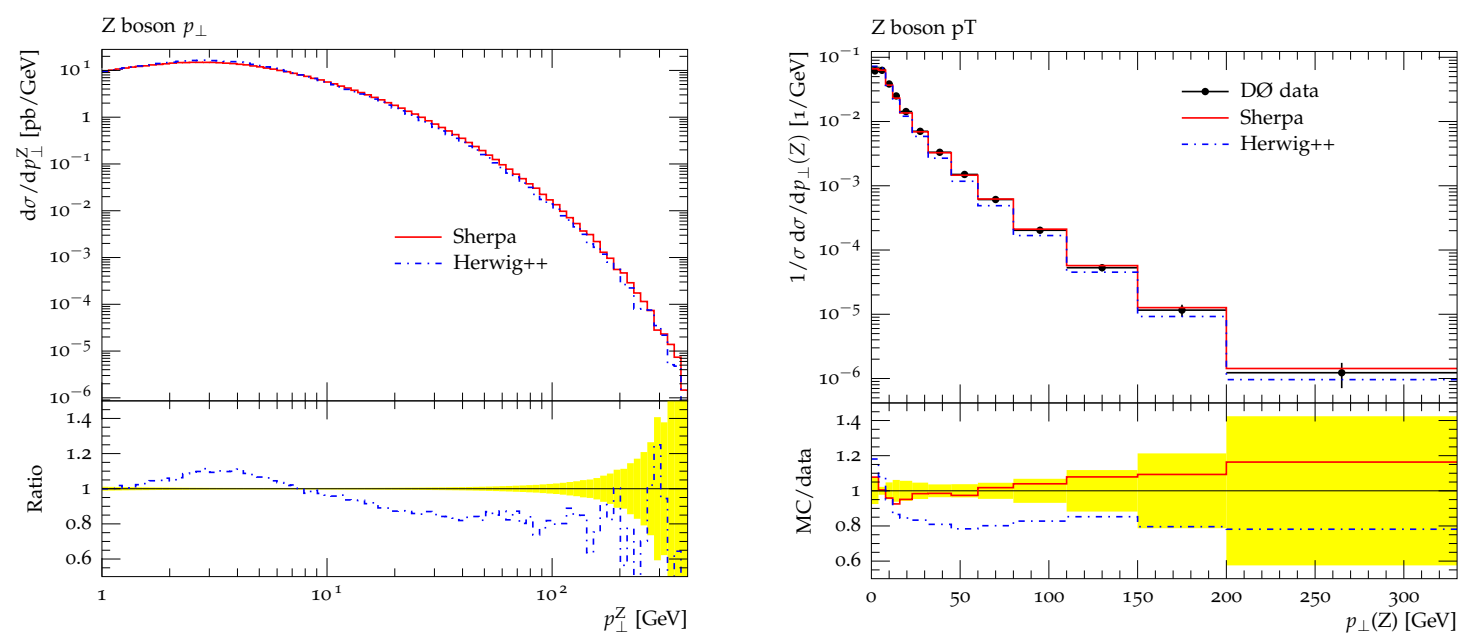

Figure 23. Transverse momentum of the lepton pair in $Z / \gamma^{*}$ production at the Tevatron.

- the stability of cross sections, as exhibited in tables 3 and 4;

- the radiation patterns, through comparison with a fake PowHEg algorithm, based on shower kernels, cf. figure 2;

- the automated detection of Born zeroes and their stable cure, as shown in figures 3 and 4 ;

- merged LO samples, see Figs 5 and 6;

- an independent Powheg implementation, Herwig++ 2.4.2, cf. figures 21-29;

- and comparison with a variety of data, cf. figures 15-20. 

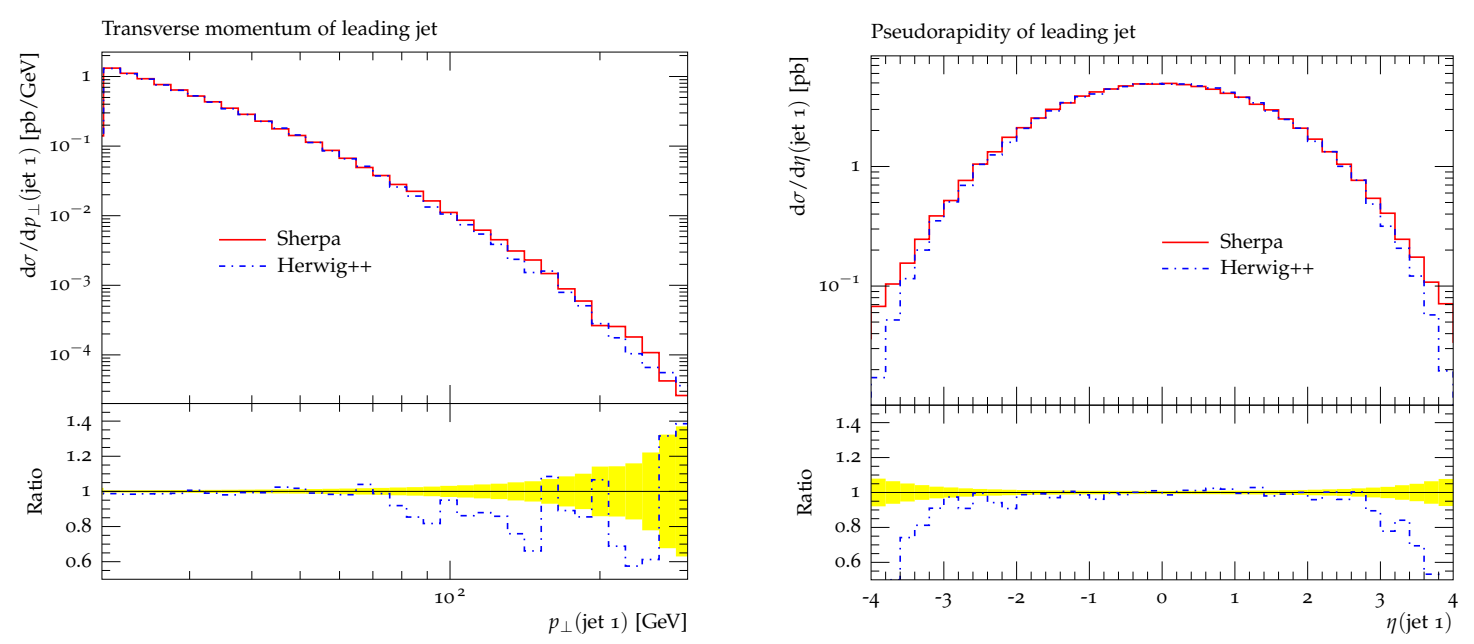

Figure 24. Transverse momentum (left) and pseudorapidity (right) of the leading jet in $Z / \gamma^{*}$ production at the Tevatron.
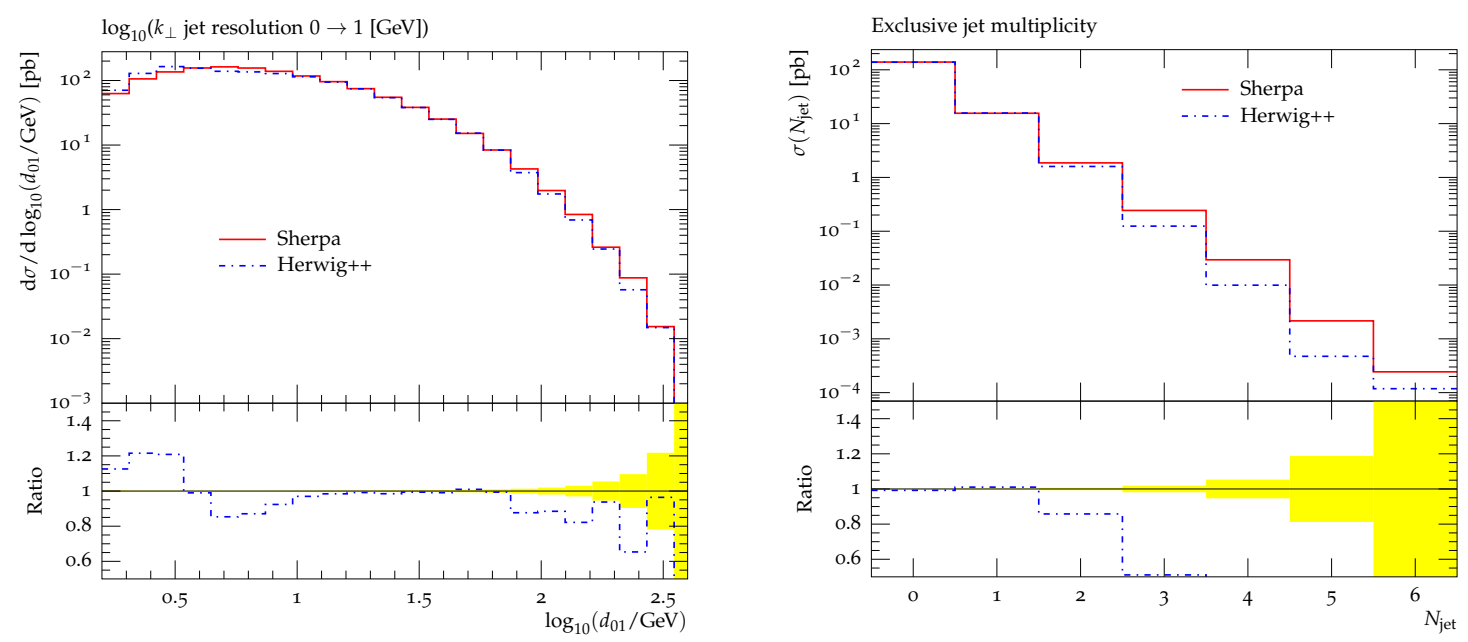

Figure 25. Predictions for the $0 \rightarrow 1$ jet resolution in $k_{T}$ clustered jets (left) and the exclusive jet multiplicity (right) in $Z / \gamma^{*}$ production at the Tevatron.

The process of $W$-pair production has been investigated for the first time using the Powheg technique. Results for this, along with some distributions in $Z$-pair production are displayed in figures 11-14.

In the near future, more processes with one coloured line only, such as $W H$ and $Z H$ associated production, will be added to the SHERPA framework and in a future publication we will also discuss the slightly more subtle treatment of processes with more than one coloured line. Furthermore, the methods developed in this publication have been used to generate merged samples with an inclusive cross section at NLO accuracy, according to the method already presented in [46]; we will report on our parallel development in a second publication [47]. This set of NLO-implementations will be made available in the future, in a new and extended release of SHERPA. 

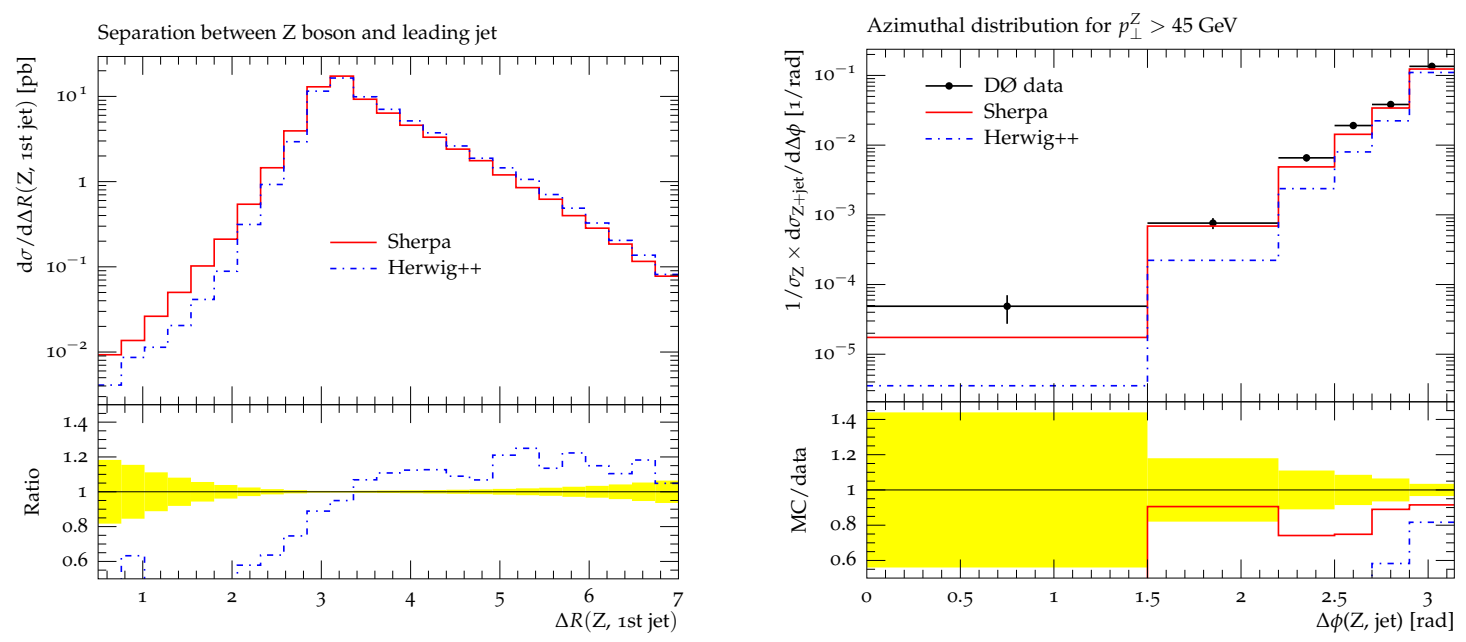

Figure 26. Separation of the leading jet and the lepton pair in $\Delta R$ (left) and $\Delta \phi$ (right) in $Z / \gamma^{*}$ production at the Tevatron.
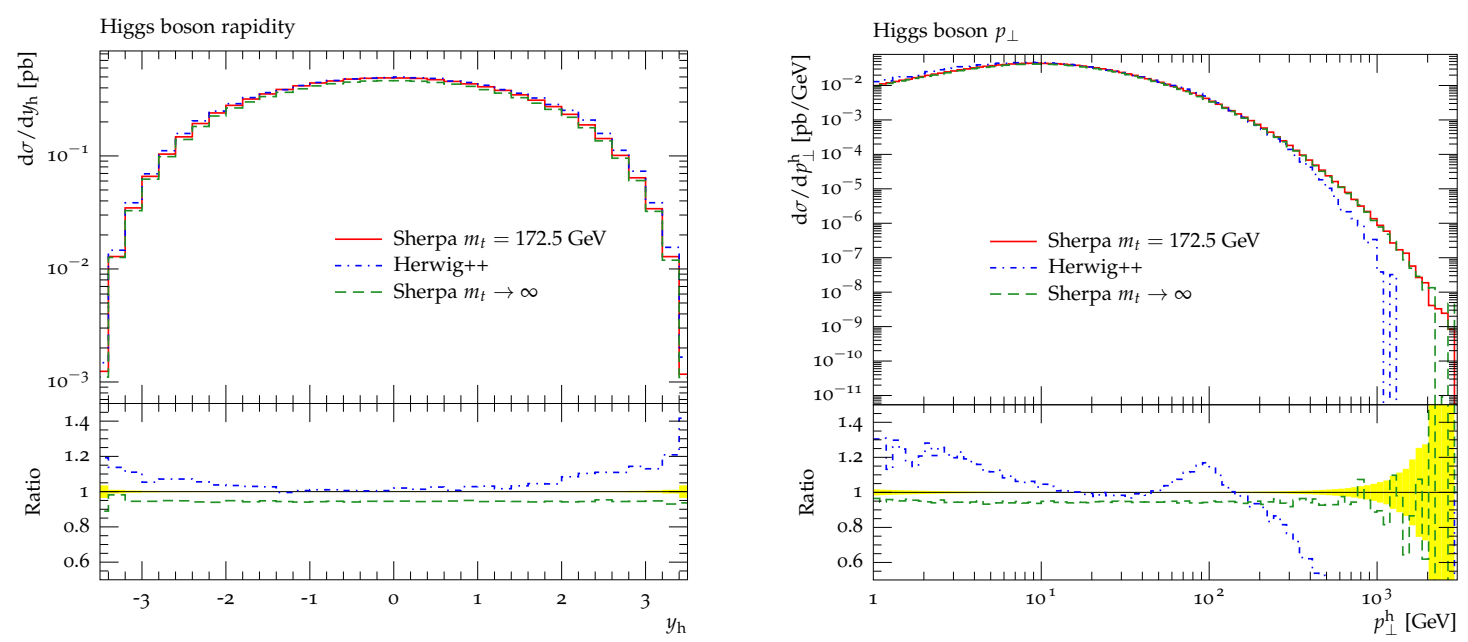

Figure 27. Rapidity (left) and transverse momentum (right) of the reconstructed Higgs boson in $g g \rightarrow h$ fusion at nominal LHC energies.

\section{Acknowledgments}

We would like to thank Jennifer Archibald, Tanju Gleisberg, Steffen Schumann and Jan Winter for many years of collaboration on the SHERPA project, and for interesting discussions. We are indebted to Daniel Maitre for support with linking the BlackHat code and for useful conversation. We thank Keith Ellis for his help in interfacing the MCFM library to Sherpa. Special thanks go to Thomas Gehrmann, Thomas Binoth and Gudrun Heinrich for numerous fruitful discussions on NLO calculations. We also thank Thomas Gehrmann for valuable comments on the manuscript. 

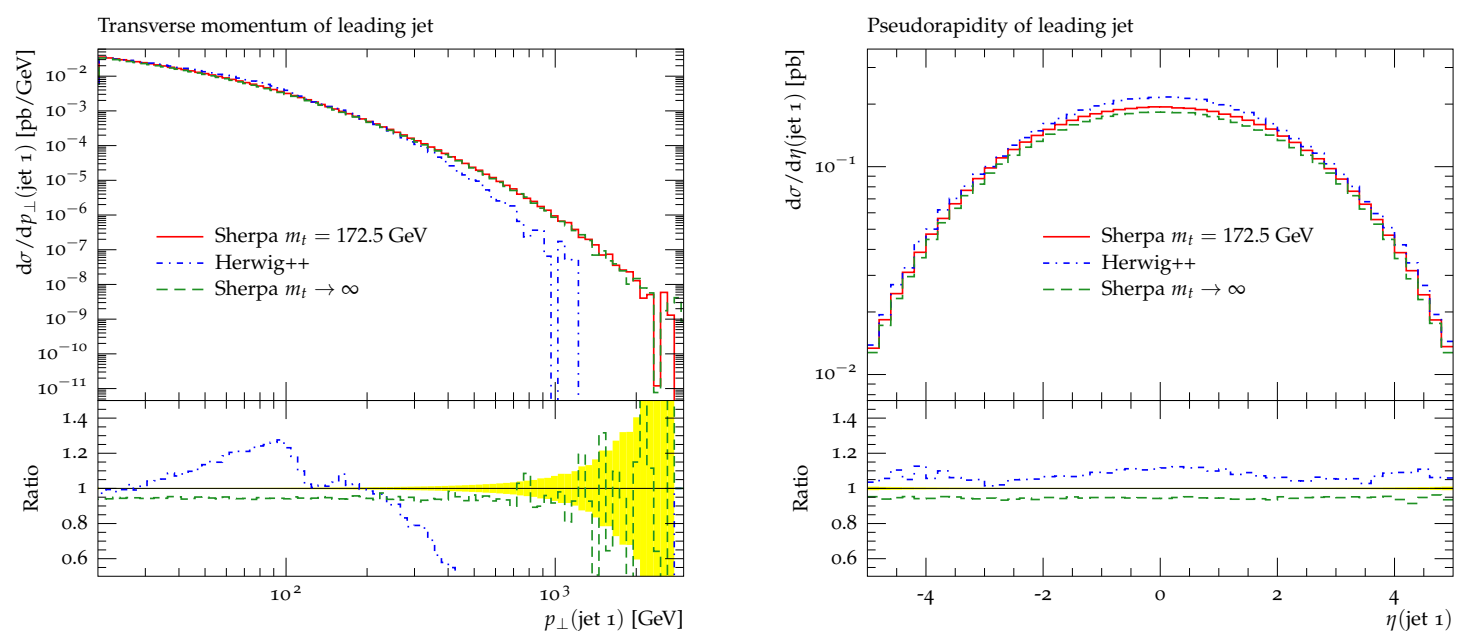

Figure 28. Transverse momentum (left) and pseudorapidity of the leading jet in $g g \rightarrow h$ fusion at nominal LHC energies.
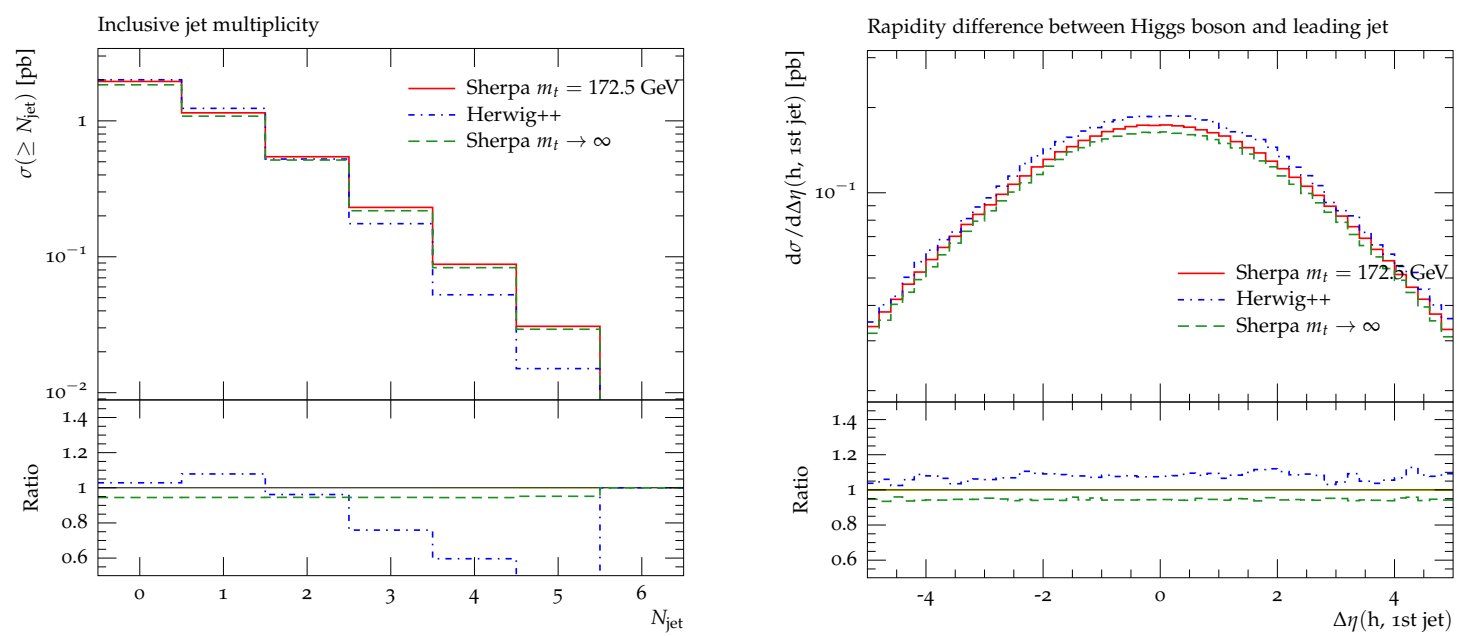

Figure 29. Inclusive jet multiplicity (left) and pseudorapidity separation of the leading jet to the reconstructed Higgs boson in $g g \rightarrow h$ fusion at nominal LHC energies.

SH acknowledges funding by the Swiss National Science Foundation (SNF, contract number 200020-126691) and by the University of Zurich (Forschungskredit number 57183003). MS and FS gratefully acknowledge financial support by the MCnet Marie Curie Research Training Network (contract number MRTN-CT-2006-035606). MS further acknowledges financial support by the HEPTOOLS Marie Curie Research Training Network (contract number MRTN-CT-2006-035505) and funding by the DFG Graduate College 1504. FK would like to thank the theory group at CERN, and MS would like to thank the Institute for Particle Physics Phenomenology in Durham, respectively, for their kind hospitality during various stages of this project. 
Open Access. This article is distributed under the terms of the Creative Commons Attribution Noncommercial License which permits any noncommercial use, distribution, and reproduction in any medium, provided the original author(s) and source are credited.

\section{References}

[1] P. Nason, A new method for combining NLO QCD with shower Monte Carlo algorithms, JHEP 11 (2004) 040 [hep-ph/0409146] [SPIRES].

[2] S. Frixione, P. Nason and C. Oleari, Matching NLO QCD computations with parton shower simulations: the POWHEG method, JHEP 11 (2007) 070 [arXiv:0709.2092] [SPIRES].

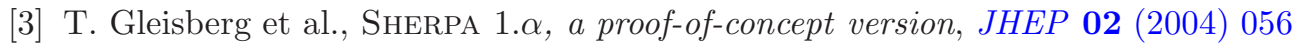
[hep-ph/0311263] [SPIRES].

[4] T. Gleisberg et al., Event generation with SHERPA 1.1, JHEP 02 (2009) 007 [arXiv:0811.4622] [SPIRES].

[5] T. Sjöstrand, S. Mrenna and P.Z. Skands, Pythia 6.4 physics and manual, JHEP 05 (2006) 026 [hep-ph/0603175] [SPIRES].

[6] T. Sjöstrand, S. Mrenna and P.Z. Skands, A brief introduction to PyтHIA 8.1, Comput. Phys. Commun. 178 (2008) 852 [arXiv:0710.3820] [SPIRES].

[7] G. Corcella et al., HeRwig 6.5: an event generator for Hadron Emission Reactions With Interfering Gluons (including supersymmetric processes), JHEP 01 (2001) 010 [hep-ph/0011363] [SPIRES].

[8] M. Bähr et al., Herwig ++ physics and manual, Eur. Phys. J. C 58 (2008) 639 [arXiv: 0803.0883] [SPIRES].

[9] J. Andre and T. Sjöstrand, A matching of matrix elements and parton showers, Phys. Rev. D 57 (1998) 5767 [hep-ph/9708390] [SPIRES].

[10] S. Catani, F. Krauss, R. Kuhn and B.R. Webber, QCD matrix elements + parton showers, JHEP 11 (2001) 063 [hep-ph/0109231] [SPIRES].

[11] F. Krauss, Matrix elements and parton showers in hadronic interactions, JHEP 08 (2002) 015 [hep-ph/0205283] [SPIRES].

[12] L. Lönnblad, Correcting the colour-dipole cascade model with fixed order matrix elements, JHEP 05 (2002) 046 [hep-ph/0112284] [SPIRES].

[13] M.L. Mangano, M. Moretti and R. Pittau, Multijet matrix elements and shower evolution in hadronic collisions: $W b \bar{b}+n$ jets as a case study, Nucl. Phys. B 632 (2002) 343 [hep-ph/0108069] [SPIRES].

[14] S. Mrenna and P. Richardson, Matching matrix elements and parton showers with HeRwiG and PythiA, JHEP 05 (2004) 040 [hep-ph/0312274] [SPIRES].

[15] M.L. Mangano, M. Moretti, F. Piccinini and M. Treccani, Matching matrix elements and shower evolution for top-quark production in hadronic collisions, JHEP 01 (2007) 013 [hep-ph/0611129] [SPIRES].

[16] J. Alwall, S. de Visscher and F. Maltoni, QCD radiation in the production of heavy colored particles at the LHC, JHEP 02 (2009) 017 [arXiv:0810.5350] [SPIRES]. 
[17] S. Höche, F. Krauss, S. Schumann and F. Siegert, QCD matrix elements and truncated showers, JHEP 05 (2009) 053 [arXiv:0903.1219] [SPIRES].

[18] K. Hamilton, P. Richardson and J. Tully, A modified CKKW matrix element merging approach to angular-ordered parton showers, JHEP 11 (2009) 038 [arXiv:0905.3072] [SPIRES].

[19] S. Höche et al., Matching parton showers and matrix elements, hep-ph/0602031 [SPIRES].

[20] J. Alwall et al., Comparative study of various algorithms for the merging of parton showers and matrix elements in hadronic collisions, Eur. Phys. J. C 53 (2008) 473 [arXiv: 0706. 2569] [SPIRES].

[21] S. Frixione and B.R. Webber, Matching NLO QCD computations and parton shower simulations, JHEP 06 (2002) 029 [hep-ph/0204244] [SPIRES].

[22] S. Frixione, P. Nason and B.R. Webber, Matching NLO QCD and parton showers in heavy flavour production, JHEP 08 (2003) 007 [hep-ph/0305252] [SPIRES].

[23] S. Frixione, E. Laenen, P. Motylinski and B.R. Webber, Single-top production in Mc@NLO, JHEP 03 (2006) 092 [hep-ph/0512250] [SPIRES].

[24] S. Frixione, E. Laenen, P. Motylinski, B.R. Webber and C.D. White, Single-top hadroproduction in association with a W boson, JHEP 07 (2008) 029 [arXiv:0805.3067] [SPIRES].

[25] C. Weydert et al., Charged Higgs boson production in association with a top quark in Mc@Nlo, Eur. Phys. J. C 67 (2010) 617 [arXiv:0912.3430] [SPIRES].

[26] P. Torrielli and S. Frixione, Matching NLO QCD computations with PyTHIA using MC@NLO, JHEP 04 (2010) 110 [arXiv:1002.4293] [SPIRES].

[27] P. Nason and G. Ridolfi, A positive-weight next-to-leading-order Monte Carlo for Z pair hadroproduction, JHEP 08 (2006) 077 [hep-ph/0606275] [SPIRES].

[28] S. Frixione, P. Nason and G. Ridolfi, A positive-weight next-to-leading-order Monte Carlo for heavy flavour hadroproduction, JHEP 09 (2007) 126 [arXiv:0707.3088] [SPIRES].

[29] S. Alioli, P. Nason, C. Oleari and E. Re, NLO Higgs boson production via gluon fusion matched with shower in POWHEG, JHEP 04 (2009) 002 [arXiv: 0812.0578] [SPIRES].

[30] S. Alioli, P. Nason, C. Oleari and E. Re, NLO single-top production matched with shower in Powheg: s- and t-channel contributions, JHEP 09 (2009) 111 [Erratum ibid. 02 (2010) 011] [arXiv:0907.4076] [SPIRES].

[31] P. Nason and C. Oleari, NLO Higgs boson production via vector-boson fusion matched with shower in POWHEG, JHEP 02 (2010) 037 [arXiv:0911.5299] [SPIRES].

[32] S. Alioli, P. Nason, C. Oleari and E. Re, NLO vector-boson production matched with shower in POWHeg, JHEP 07 (2008) 060 [arXiv: 0805.4802] [SPIRES].

[33] O. Latunde-Dada, S. Gieseke and B. Webber, A positive-weight next-to-leading-order Monte Carlo for $e^{+} e^{-}$annihilation to hadrons, JHEP 02 (2007) 051 [hep-ph/0612281] [SPIRES].

[34] O. Latunde-Dada, Applying the Powheg method to top pair production and decays at the ILC, Eur. Phys. J. C 58 (2008) 543 [arXiv:0806.4560] [SPIRES].

[35] K. Hamilton, P. Richardson and J. Tully, A positive-weight next-to-leading order Monte Carlo simulation of Drell-Yan vector boson production, JHEP 10 (2008) 015 [arXiv : 0806.0290] [SPIRES]. 
[36] A. Papaefstathiou and O. Latunde-Dada, NLO production of W-prime bosons at hadron colliders using the MC@NLO and POWHEG methods, JHEP 07 (2009) 044 [arXiv:0901.3685] [SPIRES].

[37] K. Hamilton, P. Richardson and J. Tully, A positive-weight next-to-leading order Monte Carlo simulation for Higgs boson production, JHEP 04 (2009) 116 [arXiv:0903.4345] [SPIRES].

[38] M.H. Seymour, Matrix element corrections to parton shower algorithms, Comp. Phys. Commun. 90 (1995) 95 [hep-ph/9410414] [SPIRES].

[39] M.H. Seymour, A simple prescription for first order corrections to quark scattering and annihilation processes, Nucl. Phys. B 436 (1995) 443 [hep-ph/9410244] [SPIRES].

[40] G. Corcella and M.H. Seymour, Matrix element corrections to parton shower simulations of heavy quark decay, Phys. Lett. B 442 (1998) 417 [hep-ph/9809451] [SPIRES].

[41] G. Miu and T. Sjöstrand, W production in an improved parton shower approach, Phys. Lett. B 449 (1999) 313 [hep-ph/9812455] [SPIRES].

[42] G. Corcella and M.H. Seymour, Initial state radiation in simulations of vector boson production at hadron colliders, Nucl. Phys. B 565 (2000) 227 [hep-ph/9908388] [SPIRES].

[43] E. Norrbin and T. Sjöstrand, QCD radiation off heavy particles, Nucl. Phys. B 603 (2001) 297 [hep-ph/0010012] [SPIRES].

[44] S. Alioli, P. Nason, C. Oleari and E. Re, A general framework for implementing NLO calculations in shower Monte Carlo programs: the PowHeg BOX, JHEP 06 (2010) 043 [arXiv: 1002 .2581] [SPIRES].

[45] N. Lavesson and L. Lönnblad, Extending CKKW-merging to one-loop matrix elements, JHEP 12 (2008) 070 [arXiv:0811.2912] [SPIRES].

[46] K. Hamilton and P. Nason, Improving NLO-parton shower matched simulations with higher order matrix elements, JHEP 06 (2010) 039 [arXiv: 1004.1764] [SPIRES].

[47] S. Höche, F. Krauss, M. Schönherr and F. Siegert, NLO matrix elements and truncated showers, arXiv: 1009.1127 [SPIRES].

[48] S. Catani and M.H. Seymour, A general algorithm for calculating jet cross sections in NLO QCD, Nucl. Phys. B 485 (1997) 291 [Erratum ibid. B 510 (1998) 503] [hep-ph/9605323] [SPIRES].

[49] S. Catani, S. Dittmaier, M.H. Seymour and Z. Trócsányi, The dipole formalism for next-to-leading order QCD calculations with massive partons,

Nucl. Phys. B 627 (2002) 189 [hep-ph/0201036] [SPIRES].

[50] C.F. Berger et al., An automated implementation of on-shell methods for one-loop amplitudes, Phys. Rev. D 78 (2008) 036003 [arXiv:0803.4180] [SPIRES].

[51] C.F. Berger et al., Precise predictions for $W+3$ jet production at hadron colliders, Phys. Rev. Lett. 102 (2009) 222001 [arXiv:0902.2760] [SPIRES].

[52] D. Maître, private communication.

[53] J. Campbell and R.K. Ellis, McFM - Monte Carlo for FeMtobarn processes, http://mcfm.fnal.gov.

[54] S. Frixione, Z. Kunszt and A. Signer, Three jet cross-sections to next-to-leading order, Nucl. Phys. B 467 (1996) 399 [hep-ph/9512328] [SPIRES]. 
[55] S. Frixione, A general approach to jet cross-sections in QCD, Nucl. Phys. B 507 (1997) 295 [hep-ph/9706545] [SPIRES].

[56] V.N. Gribov and L.N. Lipatov, Deep inelastic e p scattering in perturbation theory, Sov. J. Nucl. Phys. 15 (1972) 438 [Yad. Fiz. 15 (1972) 781] [SPIRES].

[57] L.N. Lipatov, The parton model and perturbation theory, Sov. J. Nucl. Phys. 20 (1975) 94 [Yad. Fiz. 20 (1974) 181] [SPIRES].

[58] Y.L. Dokshitzer, Calculation of the structure functions for deep inelastic scattering and $e^{+} e^{-}$annihilation by perturbation theory in quantum chromodynamics, Sov. Phys. JETP 46 (1977) 641 [Zh. Eksp. Teor. Fiz. 73 (1977) 1216] [SPIRES].

[59] G. Altarelli and G. Parisi, Asymptotic freedom in parton language, Nucl. Phys. B 126 (1977) 298 [SPIRES].

[60] G. Gustafson, Dual description of a confined color field, Phys. Lett. B 175 (1986) 453 [SPIRES].

[61] G. Gustafson and U. Pettersson, Dipole formulation of QCD cascades, Nucl. Phys. B 306 (1988) 746 [SPIRES].

[62] B. Andersson, G. Gustafson and L. Lönnblad, Gluon splitting in the color dipole cascades, Nucl. Phys. B 339 (1990) 393 [SPIRES].

[63] D.A. Kosower, Antenna factorization of gauge-theory amplitudes, Phys. Rev. D 57 (1998) 5410 [hep-ph/9710213] [SPIRES].

[64] D.A. Kosower, Antenna factorization in strongly-ordered limits, Phys. Rev. D 71 (2005) 045016 [hep-ph/0311272] [SPIRES].

[65] A. Gehrmann-De Ridder, T. Gehrmann and E.W.N. Glover, Antenna subtraction at NNLO, JHEP 09 (2005) 056 [hep-ph/0505111] [SPIRES].

[66] A. Daleo, T. Gehrmann and D. Maître, Antenna subtraction with hadronic initial states, JHEP 04 (2007) 016 [hep-ph/0612257] [SPIRES].

[67] S. Schumann and F. Krauss, A parton shower algorithm based on Catani-Seymour dipole factorisation, JHEP 03 (2008) 038 [arXiv:0709.1027] [SPIRES].

[68] M. Dinsdale, M. Ternick and S. Weinzierl, Parton showers from the dipole formalism, Phys. Rev. D 76 (2007) 094003 [arXiv:0709.1026] [SPIRES].

[69] W.T. Giele, D.A. Kosower and P.Z. Skands, A simple shower and matching algorithm, Phys. Rev. D 78 (2008) 014026 [arXiv:0707.3652] [SPIRES].

[70] R.D. Field, Applications of perturbative QCD, Front. Phys. 77 (1989) 1, Addison-Wesley, Redwood City U.S.A. (1989) [SPIRES].

[71] R.K. Ellis, W.J. Stirling and B.R. Webber, $Q C D$ and collider physics, first edition, Camb. Monogr. Part. Phys. Nucl. Phys. Cosmol. 8 (1996) 1 [SPIRES].

[72] S. Plätzer and S. Gieseke, Coherent parton showers with local recoils, JHEP 01 (2011) 024 [arXiv: 0909.5593] [SPIRES].

[73] S. Höche, S. Schumann and F. Siegert, Hard photon production and matrix-element parton-shower merging, Phys. Rev. D 81 (2010) 034026 [arXiv:0912.3501] [SPIRES].

[74] M. Schönherr and F. Krauss, Soft photon radiation in particle decays in SHERPA, JHEP 12 (2008) 018 [arXiv:0810.5071] [SPIRES]. 
[75] F. Krauss, R. Kuhn and G. Soff, Amegic++ 1.0: A Matrix Element Generator In $\mathrm{C}++$, JHEP 02 (2002) 044 [hep-ph/0109036] [SPIRES].

[76] T. Gleisberg and S. Höche, ComIx, a new matrix element generator, JHEP 12 (2008) 039 [arXiv:0808.3674] [SPIRES].

[77] T. Binoth et al., A proposal for a standard interface between Monte Carlo tools and one-loop programs, Comput. Phys. Commun. 181 (2010) 1612 [arXiv:1001.1307] [SPIRES].

[78] T. Gleisberg and F. Krauss, Automating dipole subtraction for QCD NLO calculations, Eur. Phys. J. C 53 (2008) 501 [arXiv:0709.2881] [SPIRES].

[79] R. Kleiss and W.J. Stirling, Spinor techniques for calculating $p \bar{p} \rightarrow W^{ \pm} / Z^{0}+$ jets, Nucl. Phys. B 262 (1985) 235 [SPIRES].

[80] A. Ballestrero and E. Maina, A new method for helicity calculations, Phys. Lett. B 350 (1995) 225 [hep-ph/9403244] [SPIRES].

[81] R. Kleiss and R. Pittau, Weight optimization in multichannel Monte Carlo, Comput. Phys. Commun. 83 (1994) 141 [hep-ph/9405257] [SPIRES].

[82] G.P. Lepage, A new algorithm for adaptive multidimensional integration, J. Comput. Phys. 27 (1978) 192 [SPIRES].

[83] G.P. Lepage, VEGAS - an adaptive multi-dimensional integration program, CLNS-80/447, Cornell University, Ithaca U.S.A. [SPIRES].

[84] Z. Nagy and D.E. Soper, Matching parton showers to NLO computations, JHEP 10 (2005) 024 [hep-ph/0503053] [SPIRES].

[85] Z. Nagy and D.E. Soper, A new parton shower algorithm: shower evolution, matching at leading and next-to-leading order level, hep-ph/0601021 [SPIRES].

[86] T. Carli, T. Gehrmann and S. Höche, Hadronic final states in deep-inelastic scattering with Sherpa, Eur. Phys. J. C 67 (2010) 73 [arXiv:0912.3715] [SPIRES].

[87] P.M. Nadolsky et al., Implications of CTEQ global analysis for collider observables, Phys. Rev. D 78 (2008) 013004 [arXiv:0802.0007] [SPIRES].

[88] B.M. Waugh et al., HZTool and Rivet: toolkit and framework for the comparison of simulated final states and data at colliders, hep-ph/0605034 [SPIRES].

[89] A. Buckley et al., Rivet user manual, arXiv:1003.0694 [SPIRES].

[90] B. Andersson, G. Gustafson, G. Ingelman and T. Sjöstrand, Parton fragmentation and string dynamics, Phys. Rept. 97 (1983) 31 [SPIRES].

[91] B. Andersson, The Lund model, Camb. Monogr. Part. Phys. Nucl. Phys. Cosmol. 7 (1997) 1 [SPIRES].

[92] S. Dawson, Radiative corrections to Higgs boson production, Nucl. Phys. B 359 (1991) 283 [SPIRES].

[93] A. Djouadi, M. Spira and P.M. Zerwas, Production of Higgs bosons in proton colliders: QCD corrections, Phys. Lett. B 264 (1991) 440 [SPIRES].

[94] C. Anastasiou, K. Melnikov and F. Petriello, Fully differential Higgs boson production and the di-photon signal through next-to-next-to-leading order, Nucl. Phys. B 724 (2005) 197 [hep-ph/0501130] [SPIRES]. 
[95] C. Anastasiou, G. Dissertori and F. Stöckli, NNLO QCD predictions for the $H \rightarrow W W \rightarrow \ell \nu \ell \nu$ signal at the LHC, JHEP 09 (2007) 018 [arXiv:0707.2373] [SPIRES].

[96] C. Anastasiou, R. Boughezal and F. Petriello, Mixed QCD-electroweak corrections to Higgs boson production in gluon fusion, JHEP 04 (2009) 003 [arXiv:0811.3458] [SPIRES].

[97] S. Catani, D. de Florian, M. Grazzini and P. Nason, Soft-gluon resummation for Higgs boson production at hadron colliders, JHEP 07 (2003) 028 [hep-ph/0306211] [SPIRES].

[98] G. Bozzi, S. Catani, D. de Florian and M. Grazzini, Transverse-momentum resummation and the spectrum of the Higgs boson at the LHC, Nucl. Phys. B 737 (2006) 73 [hep-ph/0508068] [SPIRES].

[99] J.M. Campbell and R.K. Ellis, An update on vector boson pair production at hadron colliders, Phys. Rev. D 60 (1999) 113006 [hep-ph/9905386] [SPIRES].

[100] L.J. Dixon, Z. Kunszt and A. Signer, Helicity amplitudes for $\mathcal{O}\left(\alpha_{s}\right)$ production of $W^{+} W^{-}$, $W^{ \pm} Z, Z Z, W^{ \pm} \gamma$, or $Z \gamma$ pairs at hadron colliders, Nucl. Phys. B 531 (1998) 3 [hep-ph/9803250] [SPIRES].

[101] H1 collaboration, C. Adloff et al., Measurement and QCD analysis of jet cross sections in deep-inelastic positron proton collisions at $\sqrt{s}$ of $300 \mathrm{GeV}$, Eur. Phys. J. C 19 (2001) 289 [hep-ex/0010054] [SPIRES].

[102] H1 collaboration, C. Adloff et al., Three Jet production in deep inelastic scattering at HERA, Phys. Lett. B 515 (2001) 17 [hep-ex/0106078] [SPIRES].

[103] DØ collaboration, B. Abbott et al., Differential cross-section for $W$ boson production as a function of transverse momentum in p $\bar{p}$ collisions at $\sqrt{s}=1.8 \mathrm{TeV}$,

Phys. Lett. B 513 (2001) 292 [hep-ex/0010026] [SPIRES].

[104] ALEPH collaboration, A. Heister et al., Studies of $Q C D$ at $e^{+} e^{-}$centre-of-mass energies between $91 \mathrm{GeV}$ and $209 \mathrm{GeV}$, Eur. Phys. J. C 35 (2004) 457 [SPIRES].

[105] DØ collaboration, V.M. Abazov et al., Measurement of the normalized $Z / \gamma^{*} \rightarrow \mu^{+} \mu^{-}$ transverse momentum distribution in $p \bar{p}$ collisions at $\sqrt{s}=1.96 \mathrm{TeV}$,

Phys. Lett. B 693 (2010) 522 [arXiv: 1006.0618] [SPIRES].

[106] DØ collaboration, V.M. Abazov et al., Measurement of the shape of the boson rapidity distribution for $p \bar{p} \rightarrow Z / \gamma^{*} \rightarrow e^{+} e^{-}+X$ events produced at $\sqrt{s}$ of $1.96 \mathrm{TeV}$, Phys. Rev. D 76 (2007) 012003 [hep-ex/0702025] [SPIRES].

[107] DØ collaboration, V.M. Abazov et al., Measurement of $Z / \gamma^{*}+$ jet $+X$ angular distributions in p $\bar{p}$ collisions at $\sqrt{s}=1.96 \mathrm{TeV}$, Phys. Lett. B 682 (2010) 370 [arXiv:0907.4286] [SPIRES]. 